\title{
Performance Evaluation of the ORNL Multi-Elemental KED (MEKED) Analysis Algorithms
}

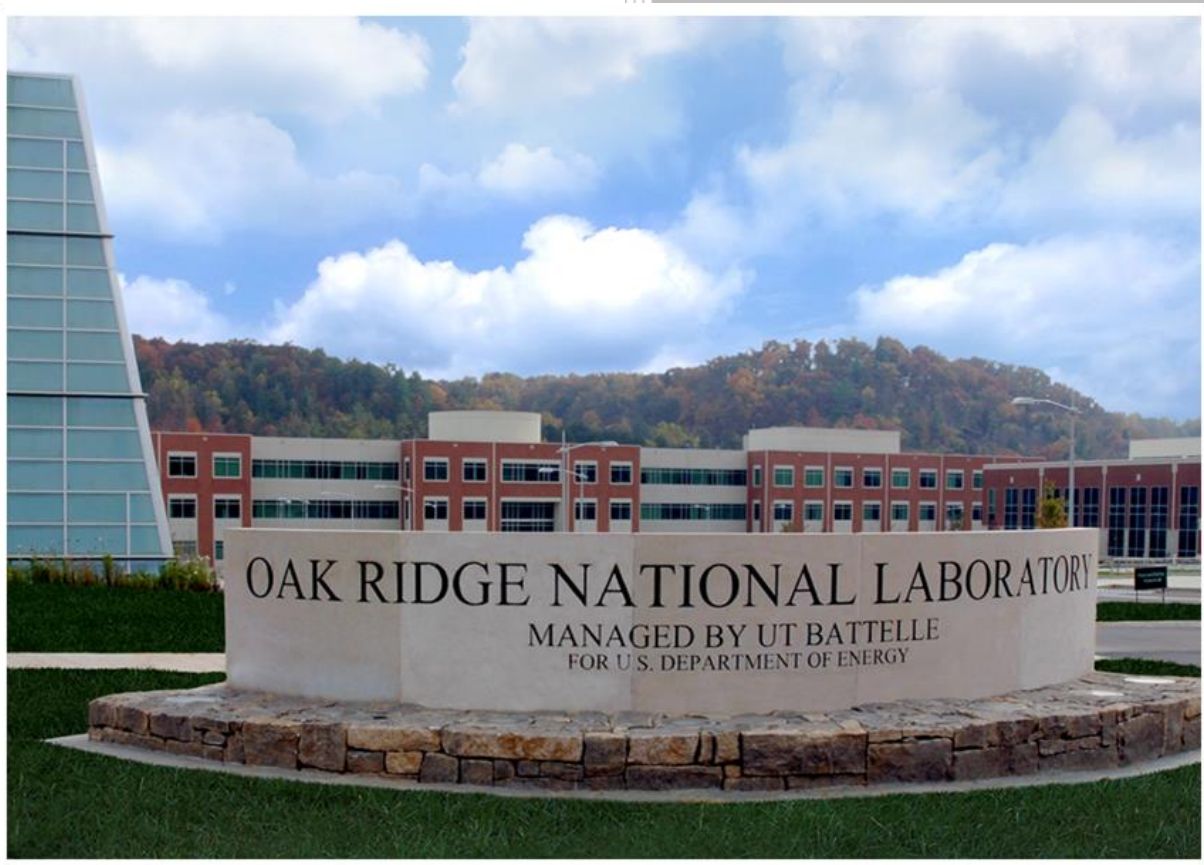

Robert D. McElroy, Jr.

October 2018 


\title{
DOCUMENT AVAILABILITY
}

Reports produced after January 1, 1996, are generally available free via US Department of Energy (DOE) SciTech Connect.

Website www.osti.gov

Reports produced before January 1, 1996, may be purchased by members of the public from the following source:

\author{
National Technical Information Service \\ 5285 Port Royal Road \\ Springfield, VA 22161 \\ Telephone 703-605-6000 (1-800-553-6847) \\ TDD 703-487-4639 \\ Fax 703-605-6900 \\ E-mail info@ntis.gov \\ Website http://classic.ntis.gov/
}

Reports are available to DOE employees, DOE contractors, Energy Technology Data Exchange representatives, and International Nuclear Information System representatives from the following source:

Office of Scientific and Technical Information

PO Box 62

Oak Ridge, TN 37831

Telephone 865-576-8401

Fax 865-576-5728

E-mail reports@osti.gov

Website http://www.osti.gov/contact.html

This report was prepared as an account of work sponsored by an agency of the United States Government. Neither the United States Government nor any agency thereof, nor any of their employees, makes any warranty, express or implied, or assumes any legal liability or responsibility for the accuracy, completeness, or usefulness of any information, apparatus, product, or process disclosed, or represents that its use would not infringe privately owned rights. Reference herein to any specific commercial product, process, or service by trade name, trademark, manufacturer, or otherwise, does not necessarily constitute or imply its endorsement, recommendation, or favoring by the United States Government or any agency thereof. The views and opinions of authors expressed herein do not necessarily state or reflect those of the United States Government or any agency thereof. 
Nuclear Security and Isotope Technology Division

\section{PERFORMANCE EVALUATION OF THE ORNL MULTI-ELEMENTAL KED (MEKED) ANALYSIS ALGORITHMS}

Robert D. McElroy, Jr.

Date Published: October 2018

Prepared by

OAK RIDGE NATIONAL LABORATORY

Oak Ridge, TN 37831-6283

managed by

UT-BATTELLE, LLC

for the

US DEPARTMENT OF ENERGY

under contract DE-AC05-00OR22725 



\section{CONTENTS}

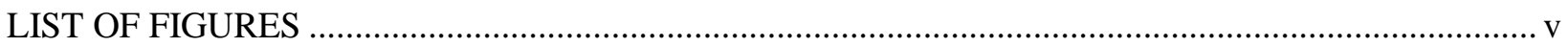

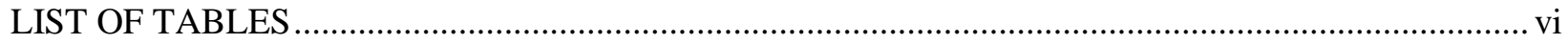

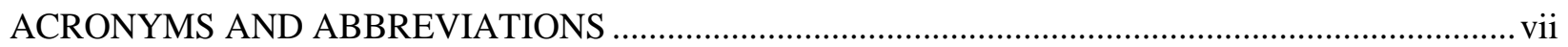

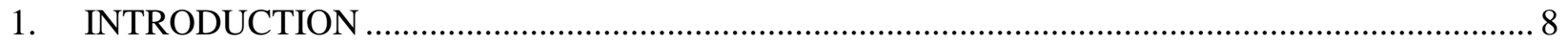

1.1 HYBRID K-EDGE/X-RAY FLUORESCENCE DENSITOMETER (HKED),...................... 8

2. LIMITATIONS OF THE TRADITIONAL ROI-BASED ANALYSIS ........................................ 9

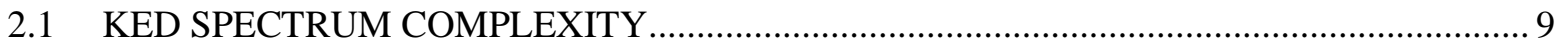

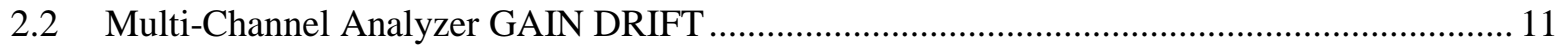

2.3 X-RAY GENERATOR END-POINT ENERGY DRIFT AND TUBE AGING …................. 12

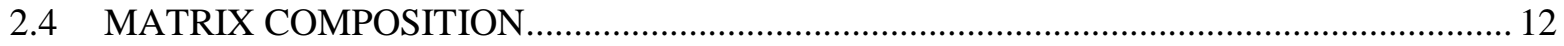

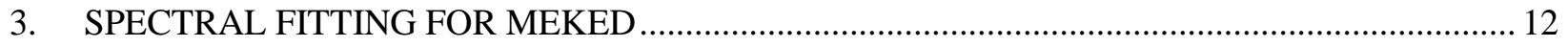

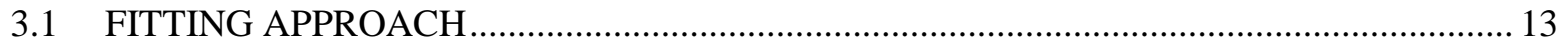

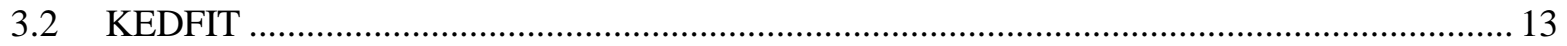

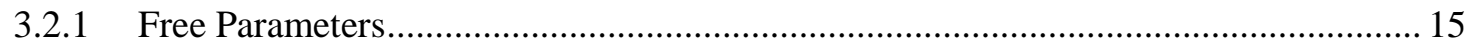

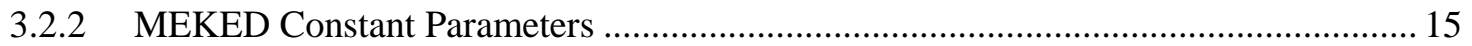

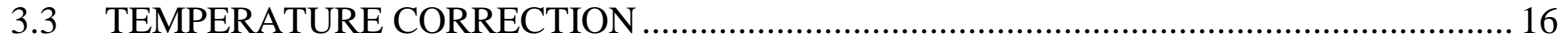

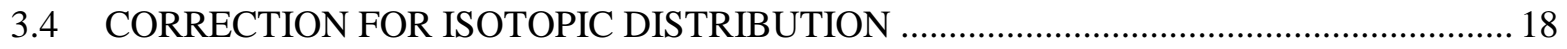

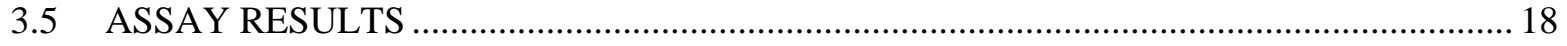

3.6 ELIMINATION OF THE MEASURED REFERENCE SPECTRUM................................ 19

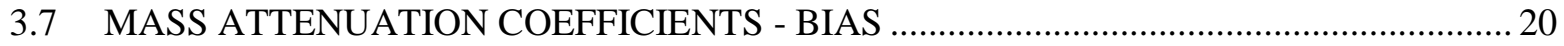

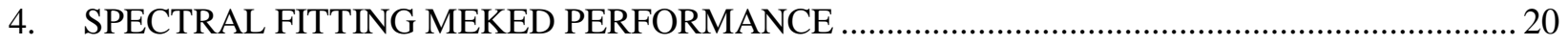

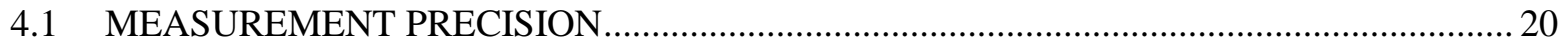

4.2 DETECTION LEVELS AND LOW CONCENTRATION SAMPLE PERFORMANCE....... 22

4.2.1 Detection Levels with Uranium as the Major Actinide ............................................. 23

5. CALCULATED VERSUS MEASURED REFERENCE SPECTRUM.......................................23

5.1 INSENSITIVITY TO X-RAY GENERATOR HV SETTINGS .......................................... 24

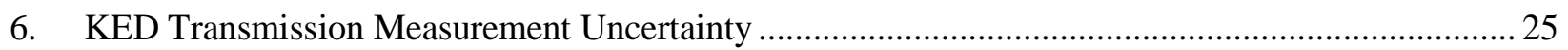

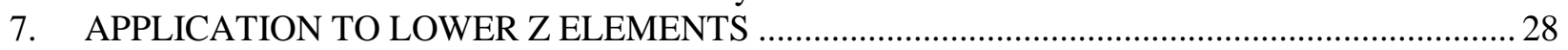

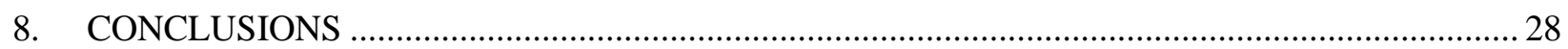

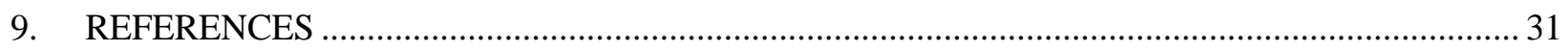

APPENDIX A. MEKED SPECTRAL RESPONSE REPRESENTATION ….................................... A-1

APPENDIX B. STANDARDS USED FOR THE ORNL HKED TESTING ....................................... B-1 



\section{LIST OF FIGURES}

Figure 1. HKED system installed at the ORNL REDC facility (left) and a sketch of the collimator, detector, and sample layout (right).

Figure 2. Plot showing the potential ROI locations (shaded areas) for a Multi-elemental KED analysis as an extension of the existing methodology.

Figure 3. The fitted KED response function and various contributors to the response superimposed on a measured spectrum for a uranium solution standard.....

Figure 4. Plot of the ratio of count rates for a KED reference spectrum and the same reference spectrum shifted 1 channel $(0.09 \mathrm{keV})$ to the right, illustrating the impact of gain shift on the KED reference spectrum.

Figure 5. Plot of a measured KED transmission spectrum from a mixed oxide (MOX) sample with the KEDFIT results (top) and the relative residual in terms of sigma (the count rate uncertainty) as a function of energy (bottom).

Figure 6. Expanded view of the "HV jitter" observed in the KED transmission spectra.

Figure 7. Sketch of the ORNL HKED system showing the approximate location within the shield housing of the thermal couple used for sample temperature measurement.

Figure 8. Reported concentration as a function of temperature showing a dependence of $0.12 \% /{ }^{\circ} \mathrm{C}$

Figure 9. Fit to a KED spectrum from a $4 M$ nitric acid reference solution.

Figure 10. Measurement precision for the spectral fit MEKED analysis and the traditional ROIbased KED analysis (1,000 $\mathrm{s}$ acquisition time)....

Figure 11. The fitted KED response function and various contributors to the response superimposed on a measured spectrum from a $322 \mathrm{~g} \mathrm{U} / \mathrm{L}$ solution standard.

Figure 12. Plots of the KED transmission spectra with fit response for vary X-ray generator settings (a: $120 \mathrm{kV}$, b: $135 \mathrm{kV}$, c: $145 \mathrm{kV}$, and d: $150 \mathrm{kV}$ ).

Figure 13. Illustration of the potential impact of a small shift in sample loading on the transmission measurement path length.

Figure 14. The measured spectra and fit response for a sample containing approximately $160 \mathrm{~g} \mathrm{Bi} / \mathrm{L}$ (left) and expanded view about the Bi K-Edge (right). . 


\section{LIST OF TABLES}

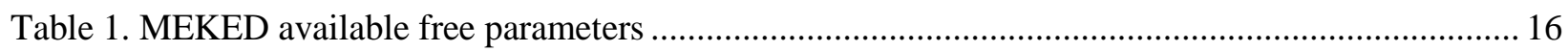

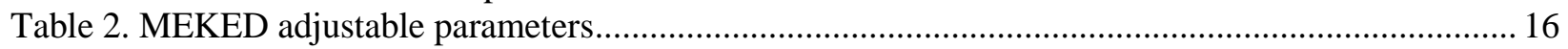

Table 3. Determination of the isotopics correction factor for typical MOX material with 28\%

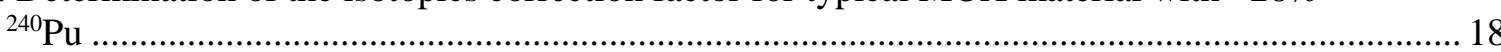

Table 4. Example assay report from the spectral fitting MEKED analysis ............................................. 19

Table 5. KED transmission precision estimates per assay segment (1,000 s/segment) for the ORNL standards [6]

Table 6. Comparison of spectral fitting MEKED MOX sample assay result with declared values (acquisition time $3 \times 1,000 \mathrm{~s}$ )

Table 7. Assay bias resulting from the MEKED K-Edge analysis of the MOX spectra provided for this study (acquisition time $3 \times 1,000 \mathrm{~s}$ )

Table 8. Comparison of the MEKED fitting results with declared values for the ORNL uranium and UPu solution standards.

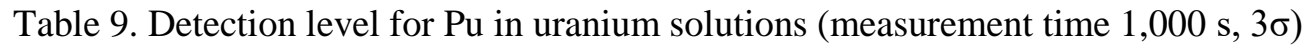
23 


\section{ACRONYMS AND ABBREVIATIONS}

$\begin{array}{ll}\text { COTS } & \begin{array}{l}\text { Commercial off the shelf } \\ \text { full width at half maximum }\end{array} \\ \text { HKED } & \text { Hybrid K-Edge Densitometer } \\ \text { HPGe } & \text { high-purity germanium } \\ \text { high voltage } \\ \text { KV } & \text { K-Edge densitometry } \\ \text { MCNP } & \text { Monte Carlo N-Particle } \\ \text { MEKED } & \text { Multi-Elemental K-Edge Densitometry } \\ \text { MEXRF } & \text { Multi-Elemental XRF } \\ \text { MOX } & \text { mixed oxide } \\ \text { ORNL } & \text { Oak Ridge National Laboratory } \\ \text { ROI } & \text { region of interest } \\ \text { XRF } & \text { X-ray fluorescence }\end{array}$




\section{INTRODUCTION}

Hybrid K-Edge Densitometer (HKED) systems integrate both K-Edge Densitometry (KED) and X-Ray Fluorescence (XRF) analyses to provide accurate, rapid assay results of the uranium and plutonium content of dissolver solution samples from nuclear fuel reprocessing facilities. Introduced for international nuclear safeguards applications in the late 1980s, the XRF component of the routine hybrid analyses is limited to quantification of $\mathrm{U}$ and $\mathrm{Pu}$ over a narrow range of $\mathrm{U}: \mathrm{Pu}$ concentration ratios of approximately 100:1. The analysis was further limited regarding the presence of minor actinide components where only a single minor actinide (typically americium) is included in the analysis and then only treated as an interference. The evolving nuclear fuel cycle has created the need to assay more complex dissolver solutions where uranium may no longer be the dominant actinide in the solution, and the concentrations of the so-called minor actinides (e.g., Th, Np, Am, and $\mathrm{Cm}$ ) are sufficiently high that they can no longer be treated as low-level impurities and ignored. Extension of the traditional HKED region of interest (ROI)-based analysis to include these additional actinides is not possible because of the increased complexity of the KED transmission spectra. Oak Ridge National Laboratory (ORNL) has developed a spectral fitting approach to the KED portion of the measurement with an enhanced algorithm set to accommodate these complex KED spectra while also eliminating the need for a reference measurement. This report provides a summary of the spectral fitting methodology and examines the performance of these algorithms using data obtained from the ORNL HKED system, as well as data provided by the International Atomic Energy Agency on actual dissolver solutions.

\subsection{HYBRID K-EDGE/X-RAY FLUORESCENCE DENSITOMETER (HKED)}

The Hybrid K-Edge/X-Ray Fluorescence Densitometer (HKED) [1] is a high-precision nondestructive assay (NDA) system developed to measure the uranium and plutonium concentrations in the aqueous dissolver and product solutions from reprocessing nuclear spent fuel (Figure 1). The HKED is a longestablished technique [2] utilized in support of International Atomic Energy Agency and European Atomic Energy Commission (EURATOM) safeguards programs. It was developed as an alternative to time-consuming destructive (chemical) analyses [1] such as isotope dilution mass spectrometry, providing uranium and plutonium concentration assays in near real time. The HKED combines K-edge densitometry with X-ray fluorescence to provide both accuracy and sensitivity for the determination of the concentrations of uranium, plutonium, and other actinides in sampled solutions across a broad range of absolute and relative concentrations (e.g., U:Pu ratios ranging from 100:1 to 1:2). When suitably calibrated, the KED subsystem determines the actinide concentrations by measuring the transmission differential from a Bremsstrahlung X-ray source (e.g., a 160-kV X-ray generator operated for 150-kV endpoint energy) across the K-shell absorption edge of the various elemental constituents of the solution. The K-shell transition energy uniquely identifies each element, and thus the K-Edge measurement is one of the few methods that can conveniently identify and distinguish these materials. The hybrid attribute, Xray fluorescence, measures the fluoresced $\mathrm{X}$-rays that are simultaneously stimulated in the sample by the impinging X-rays. Actinides have characteristic fluorescence spectra, and this additional measurement increases the capability and accuracy for characterizing the actinide samples.*

The KED is nearly absolute and the calibration is essentially for correction factors (e.g. temperature dependence) that ensure the major element is also determined with high accuracy. The XRF technique has the character of a relative measurement and again requires only correction for subtle physical effects. The

\footnotetext{
${ }^{*}$ In common use, a vial containing a few milliliters of solution is measured. The KED determines the concentration of the major element (uranium) to $<0.2 \%$ accuracy, and the XRF provides the relative concentration of the minor constituents (e.g., plutonium to an accuracy of $<0.8 \%$ ) for an assay of approximately $1 \mathrm{~h}$ in duration.
} 
HKED is a single purpose optimized blend of both techniques that has become a central plank of accountancy tank verification.
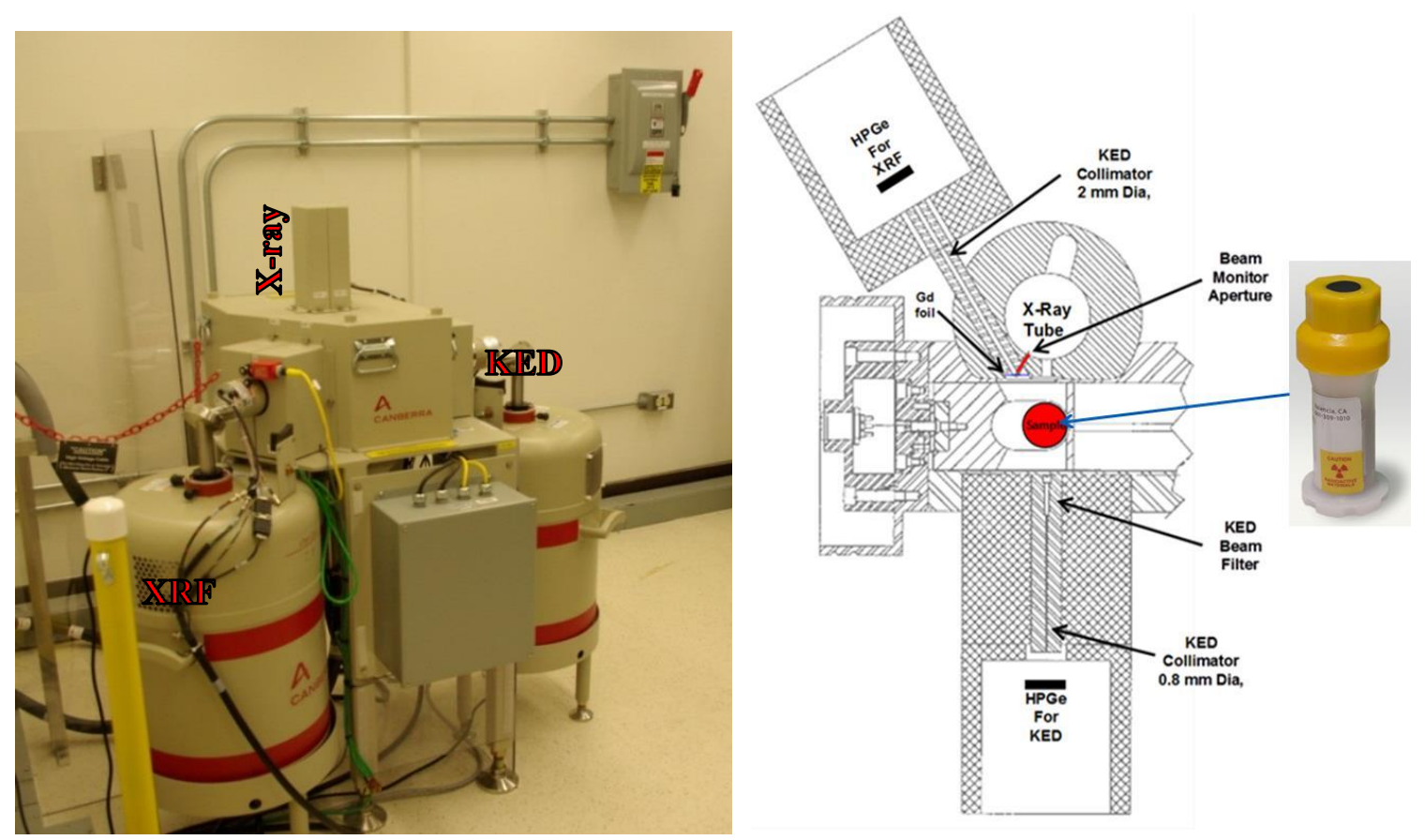

Figure 1. HKED system installed at the ORNL REDC facility (left) and a sketch of the collimator, detector, and sample layout (right).

\section{LIMITATIONS OF THE TRADITIONAL ROI-BASED ANALYSIS}

Historically, the HKED KED analysis is based on the use of simple ROI analysis [1] [3] where the total counts within a portion of the spectra are simply summed. This is an accurate and reliable approach for simple high-concentration spectra $(>10 \mathrm{~g} / \mathrm{L})$. However, for lower concentrations, the measurement uncertainty is dominated by the intense, inelastic scatter peak from the X-ray Bremsstrahlung source, and with the increasingly complex isotopic mixtures encountered in the evolving fuel cycle, the potential for interferences increases dramatically.

The primary limitation of the ROI-based analysis is that the method cannot cope with complex spectra. Stability in the X-ray generator and the spectroscopy system are also essential as small drifts in gain or interrogating X-ray energy will impact the relative count rates between the various regions of interest.

\subsection{KED SPECTRUM COMPLEXITY}

Typically, the actinides considered for a multi-elemental analysis include $\mathrm{U}, \mathrm{Np}, \mathrm{Pu}, \mathrm{Am}$, and $\mathrm{Cm}$. To determine the concentrations of these elements via KED, an ROI must be defined between their respective K-Edges. It is common practice for the ROIs to be defined outside of the instrument broadened K-Edges to minimize variation in count rate within the ROIs due to changes in electronic configuration (e.g., shaping times). The typical Gaussian peak resolution at the U K-Edge is about $525 \mathrm{eV}$ full width at half maximum (FWHM) and as good practice we would like the ROI's to begin/end at least 3 FWHM's from 
the edge or about $1.6 \mathrm{keV}$. Unfortunately, the K-Edge transitions for these elements are each separated by only about $3 \mathrm{keV}$ such that there is not enough separation between the K-Edges to allow proper setup of the measurement. A compromise would be to place the ROI limits 2 FHWM from the edges only allowing an approximate $1 \mathrm{keV}$-wide (11 channels of the spectrum) ROI between each edge (Figure 2). This is half the width currently allowed in the traditional KED measurement resulting in a $40 \%$ degradation of the measurement precision and increased sensitivity to gain drift in the high-purity germanium (HPGe) detector and increased sensitivity to shifts in the X-ray generator high voltage (HV) endpoint setting.

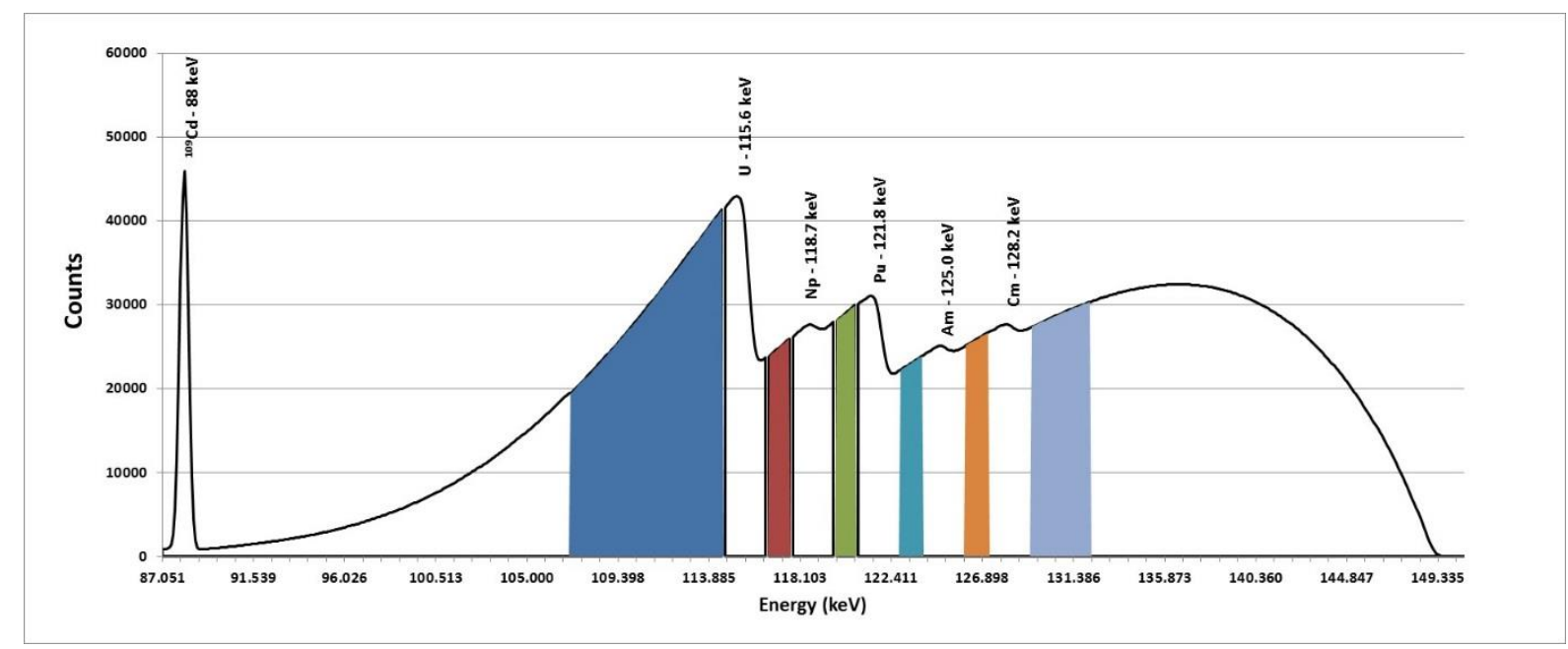

Figure 2. Plot showing the potential ROI locations (shaded areas) for a Multi-elemental KED analysis as an extension of the existing methodology.

When the effect of Lorentzian natural line width broadening of the K-Edge transitions is considered, we find that the K-Edge transitions of the $\mathrm{Z} \pm 1$ neighboring elements overlap to some extent regardless of detector resolution, so it is effectively impossible to place an ROI between the K-Edges. This interference precludes the use of an ROI methodology for the more complex actinide mixtures.

Another important consideration is how the traditional algorithms are limited by the assumption that the KED spectrum is in essence a single ratio between the measured spectrum and the reference spectrum. However, the KED spectrum cannot be considered as a simple transmission measurement over an extended energy range. As illustrated in Figure 3, the spectrum is comprised of a number of components in addition to the attenuated $\mathrm{X}$-ray source term, such as random coincidence summing, small angle scattering, detector back scattering, tailing, etc. These contributors to the transmission spectrum increase the continuum beneath the K-Edges introducing a background that is not corrected in the traditional ROI approach. The ROI methodology can only account for these components by calibration. For example, changes in the elemental composition will introduce measurement biases (e.g., calibration with $\mathrm{U}: \mathrm{Pu}=1: 1$ for assay of $\mathrm{U}: \mathrm{Pu}=2: 1$ ) by altering the relative contribution of the scattering components in the various ROIs. To obtain accurate assay results, the ROI method requires representative calibration standards spanning the expected concentrations, ranges, and elemental ratios. 


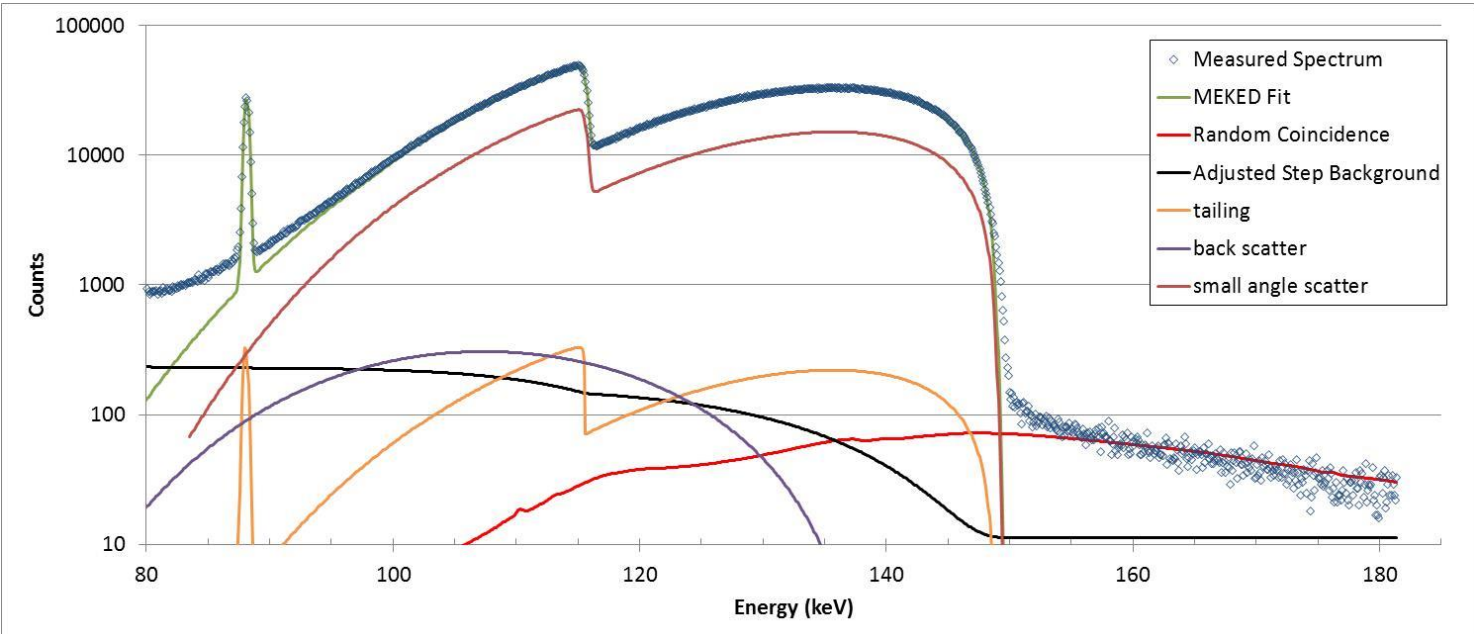

Figure 3. The fitted KED response function and various contributors to the response superimposed on a measured spectrum for a uranium solution standard.

\subsection{Multi-Channel Analyzer GAIN DRIFT}

The gain stabilization of the KED HPGe spectra over large count rate ranges (e.g., beam on/beam off) is not perfect, and from our experience it is not unreasonable to expect a 1 or 2 channel shift in the energy calibration from measurement to measurement (a typical spectrum covers 0 - $185 \mathrm{keV}$ in 2048 channels). However, because the KED spectra exhibit a rapidly varying response as a function of energy, even a one channel shift $(\sim 0.09 \mathrm{keV})$ has a significant impact on the KED assay result. This may be illustrated using the traditional ROI-based analysis where the elemental concentration is determined from a ratio of count rate ratios in the reference and sample spectra. The ratio of counts in the upper and lower ROIs about the K-Edge transition in the sample spectrum are compared with the same ratios determined for the reference spectrum.

$$
\rho=\frac{1}{\Delta \mu \cdot d} \ln \left(\frac{s_{L}}{S_{U}} \cdot \frac{R_{U}}{R_{L}}\right) \text { and } \frac{\Delta \rho}{\rho}=\frac{1}{\Delta \mu \cdot d \cdot \rho} \cdot\left(\Delta \frac{R_{U}}{R_{L}}+\Delta \frac{S_{U}}{S_{L}}\right)
$$

where $\rho$ is the concentration,

$\Delta_{\rho}$ is the change in the reported concentration due to the gain drift,

$\Delta \mu$ is the calibration coefficient $=$ change in attenuation coefficient at the K-edge transition,

$d$ is the path length,

$R_{L}$ is the count rate in the lower ROI,

$R_{U}$ is the count rate in the upper ROI,

$S_{L}$ is the count rate in the shifted spectrum lower ROI,

$S_{U}$ is the count rate in the shifted spectrum upper ROI.

The plot in Figure 4 presents the ratio of counts in the reference spectrum with the same spectrum offset by a single channel as a function of energy. The ratio of counts in the reference spectrum's lower and upper ROIs provide the baseline against which the actinide concentration is determined. The 1 channel shift in energy results in a $0.25 \%$ change in this ratio. For a sample concentration of $250 \mathrm{~g} / \mathrm{L}$, this represents a $0.2 \%$ bias in the reported actinide concentration. This uncertainty contributor is approximately as large as the random error observed for a typical 1 hour measurement. The impact of this bias will increase as the ${ }^{109} \mathrm{Cd}$ source ages and gain stabilization becomes less robust. 


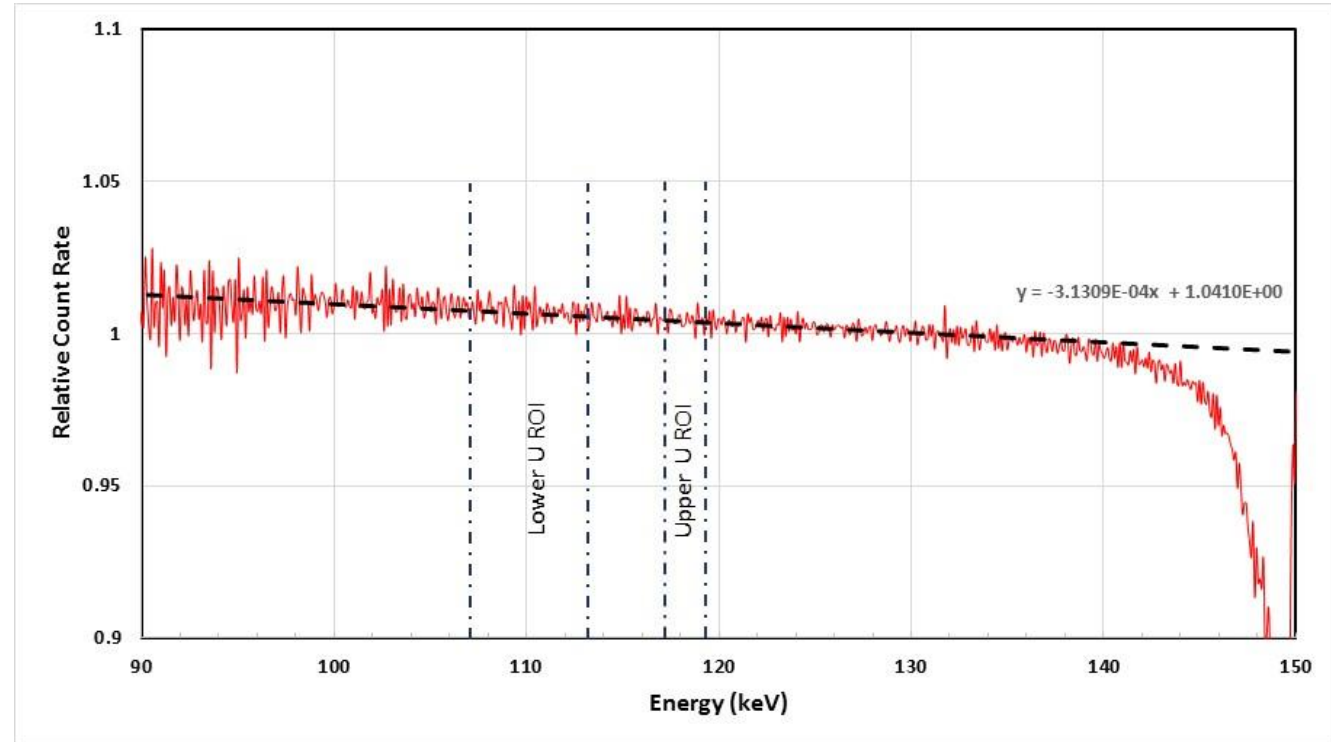

Figure 4. Plot of the ratio of count rates for a KED reference spectrum and the same reference spectrum shifted 1 channel $(0.09 \mathrm{keV})$ to the right, illustrating the impact of gain shift on the KED reference spectrum.

\subsection{X-RAY GENERATOR END-POINT ENERGY DRIFT AND TUBE AGING}

The X-ray endpoint energy can change over time as the X-ray generator and tube age. The impact of the change in endpoint energy is similar to that of the gain drift as long as the endpoint shift is small (approximately $<1 \mathrm{keV}$ ). For larger shifts in the endpoint energy, the shape of the X-ray distribution from the generator begins to change noticeably. For the 14 KED measurement data sets provided for this analysis, the average HV bias recorded by the CHKED software was $149.95 \mathrm{kV}$ with a standard deviation of $0.086 \mathrm{kV}$. This has the equivalent effect of a single channel gain shift and contributes an additional $0.2 \%$ uncertainty to the reported concentration value.

\subsection{MATRIX COMPOSITION}

We define the matrix as any material within the sample vial that is not an actinide of interest. This includes the solvent (e.g., nitric acid solution) and any nitrogen or oxygen bound to the uranium atom. For example, $4 M$ nitric acid is denser than $3 M$ nitric acid and will lead to increased attenuation of the interrogating X-rays, and if not properly accounted for, it will lead to an overreporting of the actinide concentrations. The existing VMS-based HKED and CHKED ROI-based analyses cannot detect this change in matrix composition. For the existing software packages, if such a difference is expected, then a separate calibration could be performed to correct for this difference, but only if the declaration reflects the true composition of the sample.

\section{SPECTRAL FITTING FOR MEKED}

It is clear from Figure 2 that an ROI approach cannot accommodate a complex mixture of actinides because the K-Edges from adjacent elements overlap due to both the Lorentzian broadening and the detector's response function. Reliance on a reference spectrum for the transmission measurement introduces a source of random error of equal magnitude to the statistical precision of the ROI 
measurement. To provide a more flexible, robust, and more precise analysis, a spectral fitting approach was developed for the K-Edge transmission measurement.

The spectral fitting method utilizes an analytical representation of the Bremsstrahlung spectrum emitted from the X-ray tube, applies an attenuation factor based on a description of the sample's properties and the HKED system's mechanical layout, and convolutes the resulting transmitted photon fluence with the HPGe detector response function. The approach makes use of an extended energy (87-147 keV) taking advantage of not only the K-Edge transitions but also the shape of the transmitted spectrum to determine the following:

- Concentrations of the actinides present in the solution

- The matrix (e.g., non-heavy metal constituents of the solution) concentration

- X-ray generator key parameters (e.g., HV endpoint, shaping parameter and intensity)

- Detector parameters (energy calibration, resolution, tailing parameters, backscatter component)

- Uranium and plutonium Lorentzian widths

- Thickness of the KED steel beam filter

The algorithm set used with the spectral fitting Multi-Elemental K-Edge Densitometry (MEKED) analysis is provided in Appendix A.

\subsection{FITTING APPROACH}

The MEKED analysis takes place in several steps:

1. A preliminary energy calibration is performed.

2. An initial estimate of the ${ }^{109} \mathrm{Cd}$ peak intensity is made.

3. The random coincidence contribution to the KED transmission spectrum (described in Section A.2) is subtracted. The random contribution is calculated by convolution of the spectrum with itself using either an estimated coincidence gate time (when fission products are present) or by normalization of the counts in the energy region above the X-ray generator setpoint.

4. The "step background" is removed in a manner similar to that described by Ottmar and Eberle [1], except that the magnitude of the step background is adjusted to exclude the contribution associated with ${ }^{109} \mathrm{Cd}$ source.

5. Initial parameter estimates are determined using a simplified form of the traditional VMS HKED analysis.

6. The fitting routine, KEDFIT, is launched to determine the actinide concentrations.

7. The actinide concentrations are corrected for isotopic composition and temperature effects.

\subsection{KEDFIT}

The fitting routine, KEDFIT, is based upon Bevington's CURFIT [4], which is a Levenberg-Marquardt nonlinear least squares curve-fitting routine. Minor modifications to the routine were made to improve the accuracy of the reported errors and to provide a covariance array between the model parameters. To facilitate development, the fitting approach was written in Visual Basic using Microsoft Excel as the user 
interface. (For speed and quality control purposes, it will be eventually necessary to transport the KEDFIT code to a more robust programming language such as $\mathrm{C}++$.)

The fitting process yields a high-fidelity representation of the measured KED transmission spectra. An example of a typical fit result is shown in Figure 5. The calculated response function accurately represents the measured spectrum across the energy range $(87-147 \mathrm{keV})$ of the fit. The energy range is limited to just below the $88 \mathrm{keV}$ line from ${ }^{109} \mathrm{Cd}$ to just below the $\mathrm{X}$-ray generator $\mathrm{HV}$ endpoint, which is nominally set to $150 \mathrm{kV}$. The region below the $88 \mathrm{keV}$ line is excluded to avoid the unnecessary complexity of the tungsten X-ray region.

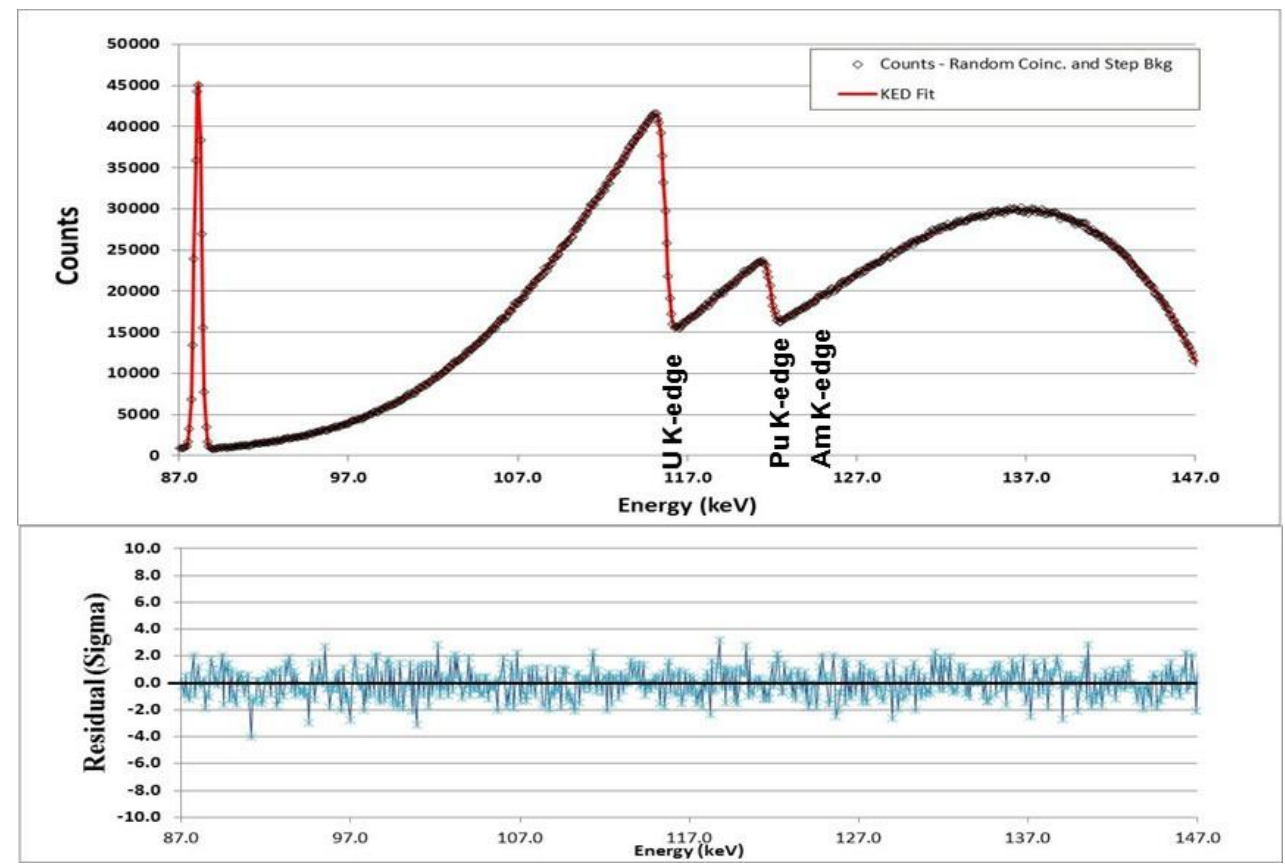

Figure 5. Plot of a measured KED transmission spectrum from a mixed oxide (MOX) sample with the KEDFIT results (top) and the relative residual in terms of sigma (the count rate uncertainty) as a function of energy (bottom).

The energy region about the X-ray endpoint energy is excluded from the fit because of an unexplained feature of the spectrum extending $\pm 3 \mathrm{kV}$ about the expected cutoff energy. This deviation is likely to be an additional pile-up feature or potentially a jitter in the X-ray generator HV supply. The deviation is illustrated in Figure 6. 


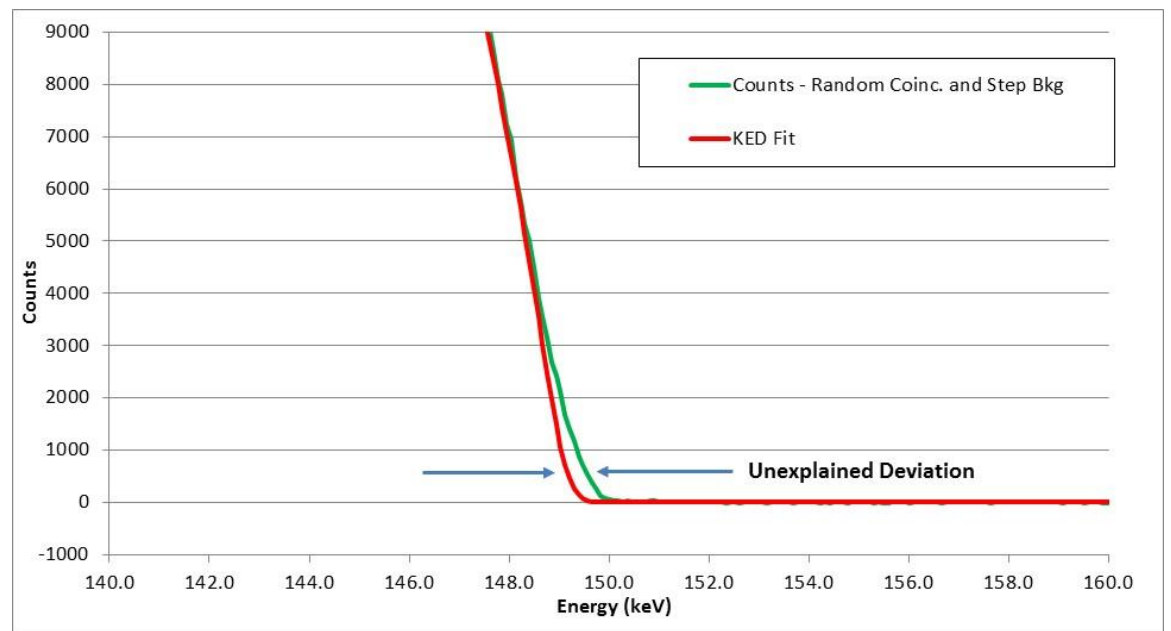

Figure 6. Expanded view of the "HV jitter" observed in the KED transmission spectra.

\subsubsection{Free Parameters}

In principle, there are as many as 27 free MEKED parameters; however, many of these parameters have been found to have an insignificant impact on the fit and are treated as constants or are determined from analysis of initial assay results - a calibration in a sense - and then treated as a constant for a given HKED system. A typical multi-elemental analysis will have 13-19 free variables that must be determined during the fitting process. The adjustable parameters are listed in Table 1. (Parameters shaded in grey are characteristic of an HKED system that are not routinely adjusted during the fitting process. These will be determined from the first few analyses with the system and then may be fixed.) Actinides not expected to be present in the solution can also be excluded from the fitting process. The function of each of these parameters is discussed in Appendix A. These parameters may be selected as free or fixed during the fitting process.

\subsubsection{MEKED Constant Parameters}

A number of additional parameters necessary for the analysis are exposed (not hard coded) to allow flexibility in the fitting process, such as the vial inner diameter and fitting energy range. A full listing of the constant parameters is provided in Table 2 with representative values for the ORNL HKED system. These will be nearly the same for all HKED systems based on the commonly used SGN vial (shown in Figure 1).

These parameters are discussed in Appendix A. Hardware parameters and sample properties are determined from system mechanical drawings and design documents. The ${ }^{109} \mathrm{Cd}$ peak correction and background normalization factors were determined from the ${ }^{109} \mathrm{Cd}$ spectrum obtained with the X-ray generator off and no sample vial present for the KED detector and will be discussed in a following section of this report. 


\begin{tabular}{|c|c|c|c|}
\hline \multicolumn{2}{|c|}{ Sample properties } & \multicolumn{2}{|c|}{ System properties } \\
\hline 1 & U Concentration $(\mathrm{g} / \mathrm{mL})$ & 19 & ${ }^{109} \mathrm{Cd}$ peak intensity \\
\hline 2 & Pu Concentration (g/mL) & 20 & ${ }^{109} \mathrm{Cd}$ peak energy $(\mathrm{keV})$ \\
\hline 3 & Np Concentration $(\mathrm{g} / \mathrm{mL})$ & 21 & ${ }^{109} \mathrm{Cd}$ step background relative intensity \\
\hline 4 & Am Concentration $(\mathrm{g} / \mathrm{mL})$ & 22 & Small angle scatter relative intensity \\
\hline 5 & Cm Concentration $(\mathrm{g} / \mathrm{mL})$ & 23 & KED collimator acceptance angle \\
\hline 6 & Matrix Density $(\mathrm{g} / \mathrm{mL})$ & 24 & Steel Beam Filter Thickness $(\mathrm{cm})$ \\
\hline \multicolumn{2}{|c|}{$\mathrm{X}$-ray generator properties } & \multicolumn{2}{|l|}{ Other } \\
\hline 7 & $\mathrm{E}_{0}, \mathrm{X}$-ray Generator HV setpoint $(\mathrm{kV})$ & 25 & Lorentzian width of U K-Edge $(\mathrm{keV})$ \\
\hline 8 & $\mathrm{I}_{0}, \mathrm{X}$-ray intensity & 26 & Lorentzian width of Pu U K-Edge (keV) \\
\hline 9 & $\mathrm{x}, \mathrm{X}$-ray source term shaping parameter & 27 & U XRF peak intensity (normally $=0$ ) \\
\hline \multicolumn{4}{|c|}{ Detector properties } \\
\hline 10 & $\mathrm{e}_{0}, \mathrm{HPGe}$ spectrum offset $(\mathrm{keV})$ & 13 & Slow Tail Area \\
\hline 11 & $\Delta \mathrm{e}, \mathrm{HGPE}$ spectrum slope (keV/channel) & 14 & Slow Tail Decay constant (keV) \\
\hline 12 & $\sigma$, Gaussian width $(\mathrm{keV})$ & 15 & Fast Tail Area \\
\hline 17 & Backscatter intensity & 16 & Fast Tail Decay constant $(\mathrm{keV})$ \\
\hline 18 & Ge escape peak relative intensity & & \\
\hline
\end{tabular}

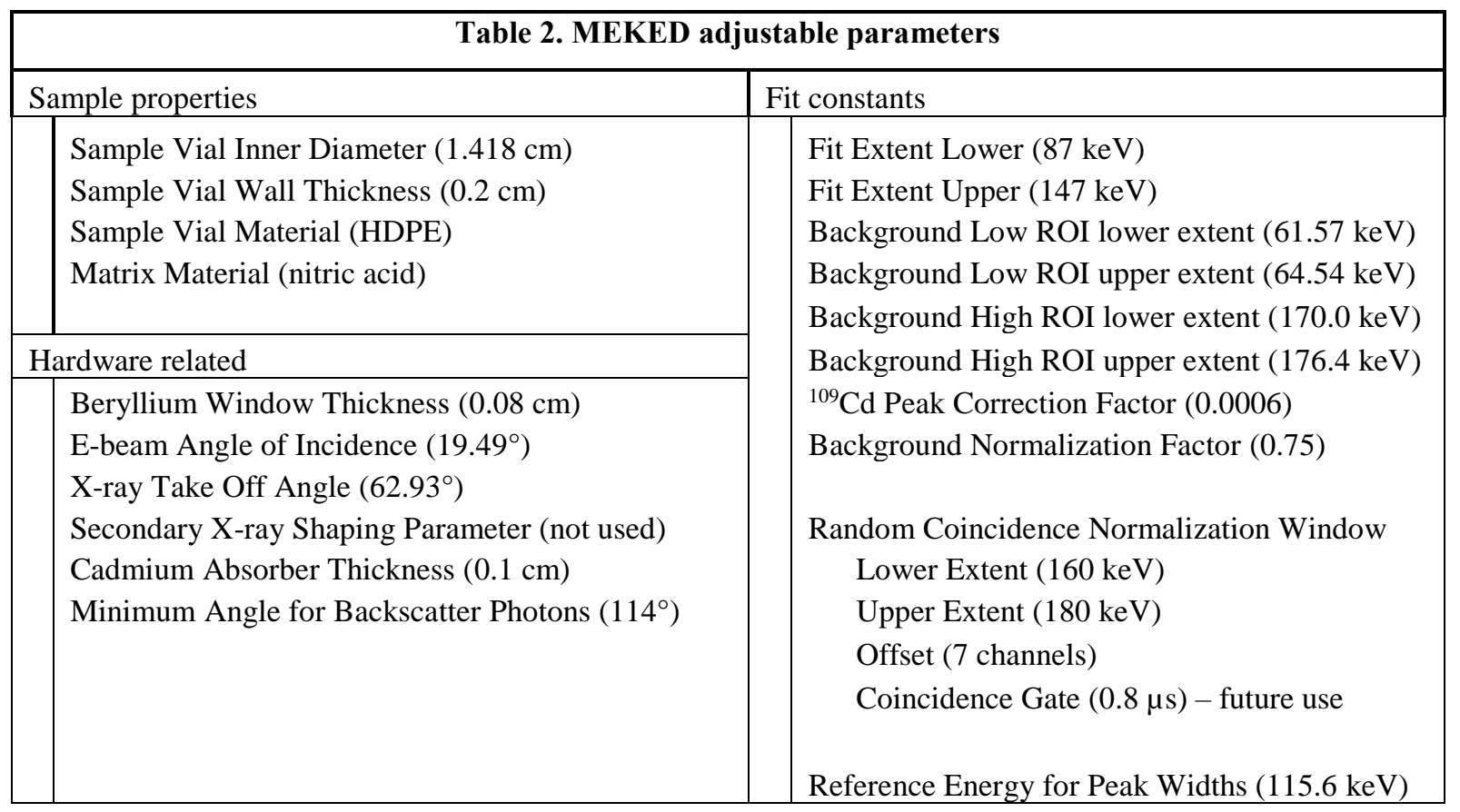

\subsection{TEMPERATURE CORRECTION}

Temperature changes in the measurement region impact not only the density of the sample solution but also the dimensions and density of the sample vial and the HKED mechanism. As the sample temperature increases, the solution density will decrease, but because the sample vial is expanding so that there is a greater volume of solution in the transmission path, it reduces the impact of the expansion of the solution. An empirical calibration is applied to correct for the temperature effect. 
As the temperature changes, the density and diameter of the KED collimator change as well as the apparent thickness of the attenuators. The primary impact of these changes is a general increase or decrease in the transmission rate, which only has a slight energy dependence. Although there seems to be a change in the scattered component of the response function, there is no impact on the fitted concentration results because the fitting process treats the beam intensity as a free parameter.

To determine the temperature dependences for the ORNL HKED system, a series of repeat measurements were performed allowing the operation of the X-ray generator to provide a slow, continuous increase in sample temperature. For these measurements, a thermocouple was placed inside the protective lead shield but just outside HKED sample transfer tube. A second thermocouple was located outside of the shield housing (Figure 7). Six assays with three 1,000 second repeat measurements were performed on a $323 \mathrm{~g} \mathrm{U} / \mathrm{L}$ sample and the reported concentrations presented in Figure 8. The measurements were conducted consecutively, and the system temperature increased smoothly throughout these measurements because of heating by operation of the X-ray generator. We find that the temperature correction for the ORNL system using the SGN vials using the spectral fitting MEKED approach is

$$
\rho_{25}=[1+0.0012 \cdot(T-25)] \cdot \rho_{T},
$$

where $\rho_{25}$ is the actinide concentration corrected to $25^{\circ} \mathrm{C}$,

$\rho_{T}$ is the concentration at the measurement temperature,

$T$ is the temperature of the sample transfer tube during measurement in ${ }^{\circ} \mathrm{C}$.

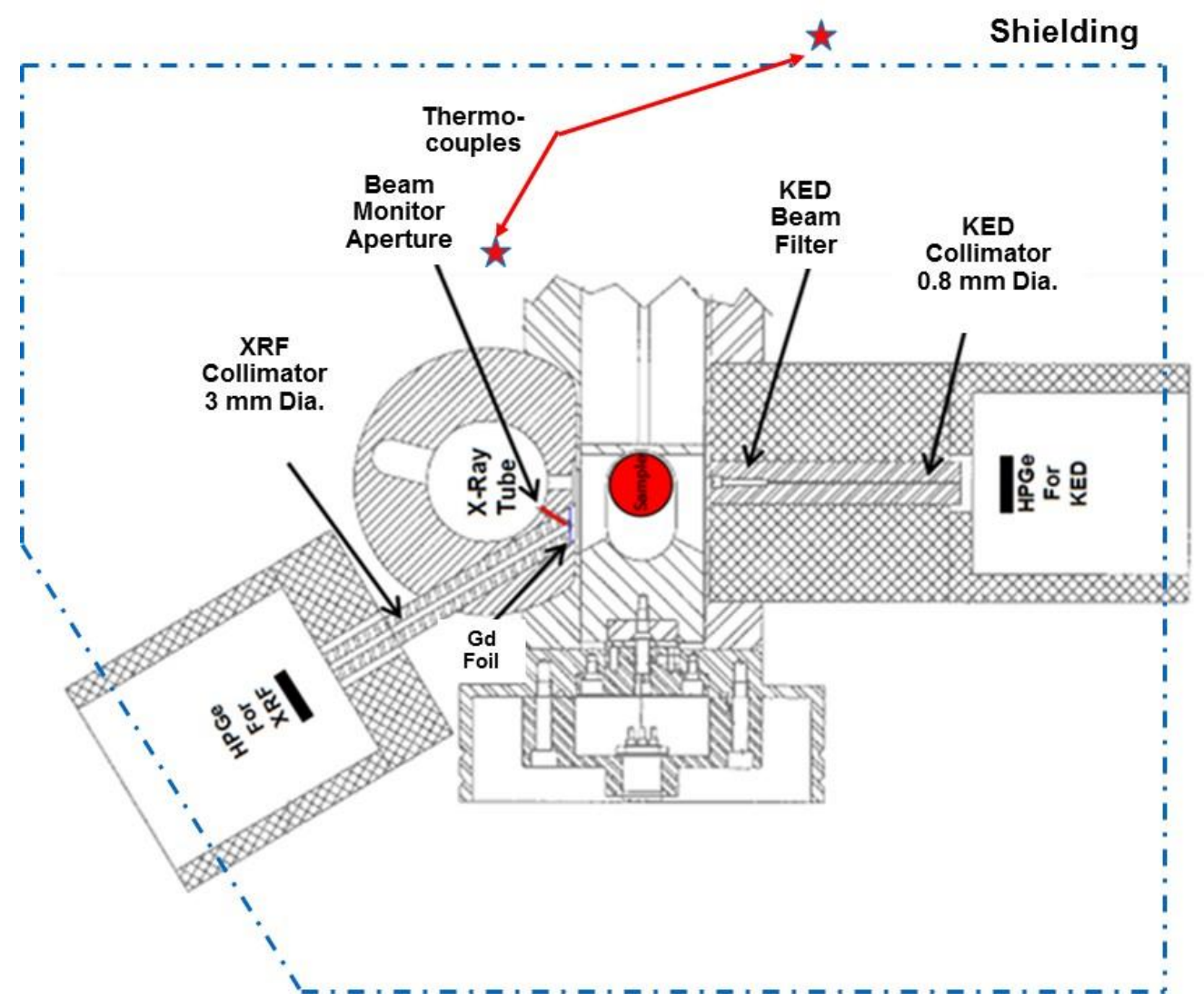

Figure 7. Sketch of the ORNL HKED system showing the approximate location within the shield housing of the thermal couple used for sample temperature measurement. 


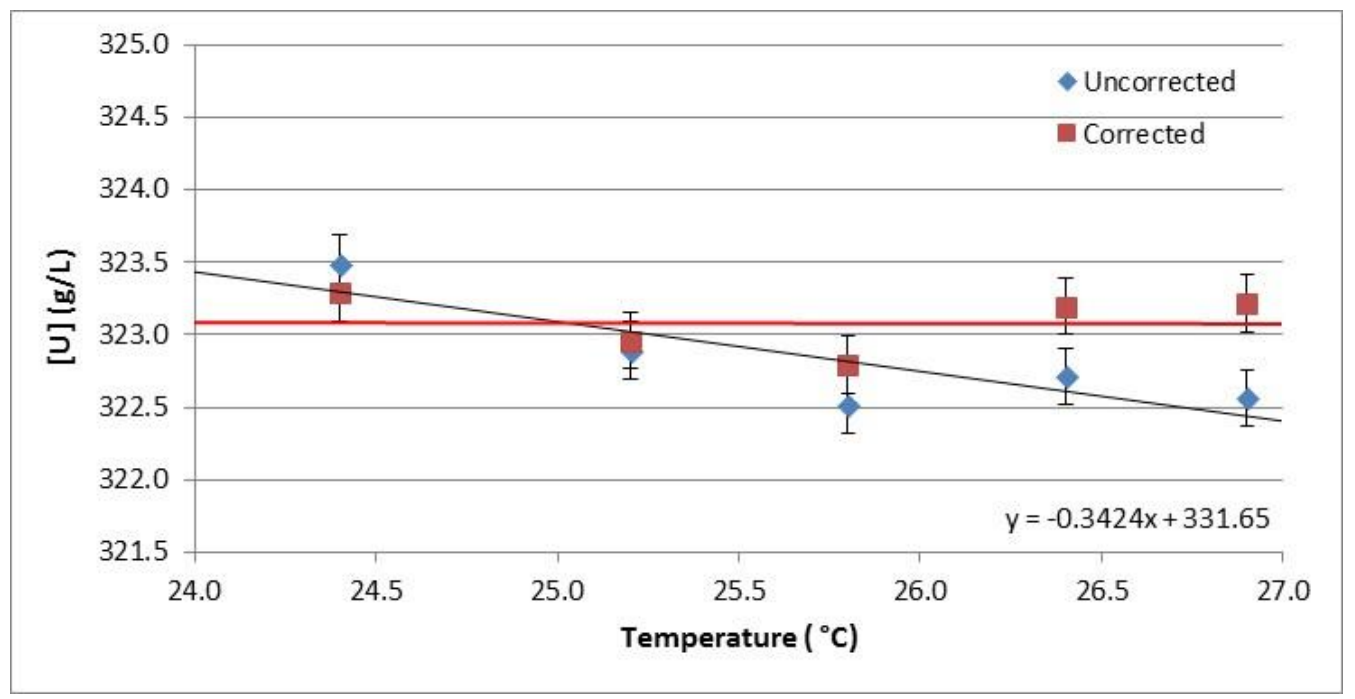

Figure 8. Reported concentration as a function of temperature showing a dependence of $0.12 \% /{ }^{\circ} \mathrm{C}$.

\subsection{CORRECTION FOR ISOTOPIC DISTRIBUTION}

The mass attenuation coefficients provided in the XCOM Photon Cross Sections Database [5] for elements with no stable isotopes are listed relative to the longest-lived isotope. The reported concentrations must be corrected for the differences in isotopic distribution. For uranium, the reference isotope is ${ }^{238} \mathrm{U}$ and for plutonium it is ${ }^{244} \mathrm{Pu}$. Since the HKED system is generally used for low-enriched, natural, or depleted uranium, the correction is small $(<0.1 \%)$; however, for plutonium the correction can be as much as a few percent. For typical MOX materials, with $\sim 28 \%{ }^{240} \mathrm{Pu}$, the correction is about $1.8 \%$ as illustrated in Table 3 . The correction for uranium is performed in a similar manner.

Table 3. Determination of the isotopics correction factor for typical MOX material with $\sim 28 \%{ }^{240} \mathrm{Pu}$

\begin{tabular}{cccc}
\hline Isotope & Atomic mass & Abundance & Partial mass \\
\hline${ }^{238} \mathrm{Pu}$ & 238.05 & $1.8 \%$ & 4.394 \\
${ }^{239} \mathrm{Pu}$ & 239.05 & $57.2 \%$ & 136.679 \\
${ }^{240} \mathrm{Pu}$ & 240.05 & $28.5 \%$ & 68.462 \\
${ }^{241} \mathrm{Pu}$ & 241.06 & $5.6 \%$ & 13.557 \\
${ }^{241} \mathrm{Pu}$ & 242.06 & $6.8 \%$ & 16.540 \\
${ }^{244} \mathrm{Pu}$ & 244.07 & $0.0 \%$ & 0.000 \\
\hline Total & & 239.633 \\
Correction factor & & 1.0185 \\
\hline
\end{tabular}

\subsection{ASSAY RESULTS}

Historically, the HKED assays are divided into three short cycles as a means to estimate the measurement precision of the result. To accommodate existing measured spectra and provide a familiar look to the analysis, the three spectra are analyzed independently, but the summed spectra results are also provided. An example output is provided in Table 4. 
Table 4. Example assay report from the spectral fitting MEKED analysis

\begin{tabular}{|c|c|c|c|c|c|c|c|c|c|c|}
\hline \multicolumn{11}{|c|}{ Atomic Weight Corrected Assay Results (g/cc) } \\
\hline & \multicolumn{2}{|c|}{ Matrix (g/L) } & \multicolumn{2}{|c|}{ Uranium $(\mathrm{g} / \mathrm{L})$} & \multicolumn{2}{|c|}{ Neptunium $(\mathrm{g} / \mathrm{L})$} & \multicolumn{2}{|c|}{ Plutonium $(\mathrm{g} / \mathrm{L})$} & \multicolumn{2}{|c|}{ Americium (g/L) } \\
\hline Rep 1 & 1.273 & \pm 0.001 & 101.53 & \pm 0.04 & 0.08 & \pm 0.04 & 9.00 & \pm 0.04 & 0.30 & \pm 0.05 \\
\hline Rep 2 & 1.244 & \pm 0.001 & 101.67 & \pm 0.04 & 0.20 & \pm 0.04 & 8.82 & \pm 0.04 & 0.14 & \pm 0.03 \\
\hline Rep 3 & 1.269 & \pm 0.001 & $\mathbf{1 0 1 . 8 3}$ & \pm 0.04 & 0.08 & \pm 0.02 & 9.22 & \pm 0.04 & 0.10 & \pm 0.02 \\
\hline Sum spectrum & 1.283 & \pm 0.000 & 101.60 & \pm 0.02 & 0.09 & \pm 0.02 & 8.92 & \pm 0.02 & 0.14 & \pm 0.03 \\
\hline Average & 1.262 & \pm 0.009 & 101.68 & \pm 0.09 & 0.12 & \pm 0.04 & 9.01 & \pm 0.12 & 0.18 & \pm 0.06 \\
\hline St. Dev. & 0.015 & & 0.15 & & 0.07 & & 0.20 & & 0.10 & \\
\hline Wt. Avg. & 1.262 & \pm 0.000 & 101.68 & \pm 0.02 & 0.09 & \pm 0.01 & 9.01 & \pm 0.02 & 0.13 & \pm 0.02 \\
\hline
\end{tabular}

\subsection{ELIMINATION OF THE MEASURED REFERENCE SPECTRUM}

One of the most significant differences between the spectral fitting approach and the ROI-based analyses is the elimination of the measured reference spectrum. As discussed in the Appendix, the fitted X-ray source term can be treated as an analytical function represented by no more than three free parameters in the fitting process (the endpoint energy, intensity, and a shaping parameter). The source term is modified by hardware configuration (e.g., beam filters thickness and material), sample container (e.g., material and dimensions), detector response, and actinide solution parameters. This representation of the HKED response encompasses the description of the reference spectrum (i.e., the spectrum from measurement of a reference blank is accurately described by these algorithms and physical parameters). To demonstrate that the reference solution is not needed with this approach, Figure 9 shows the fit response for a typical reference blank containing $4 M$ nitric acid.

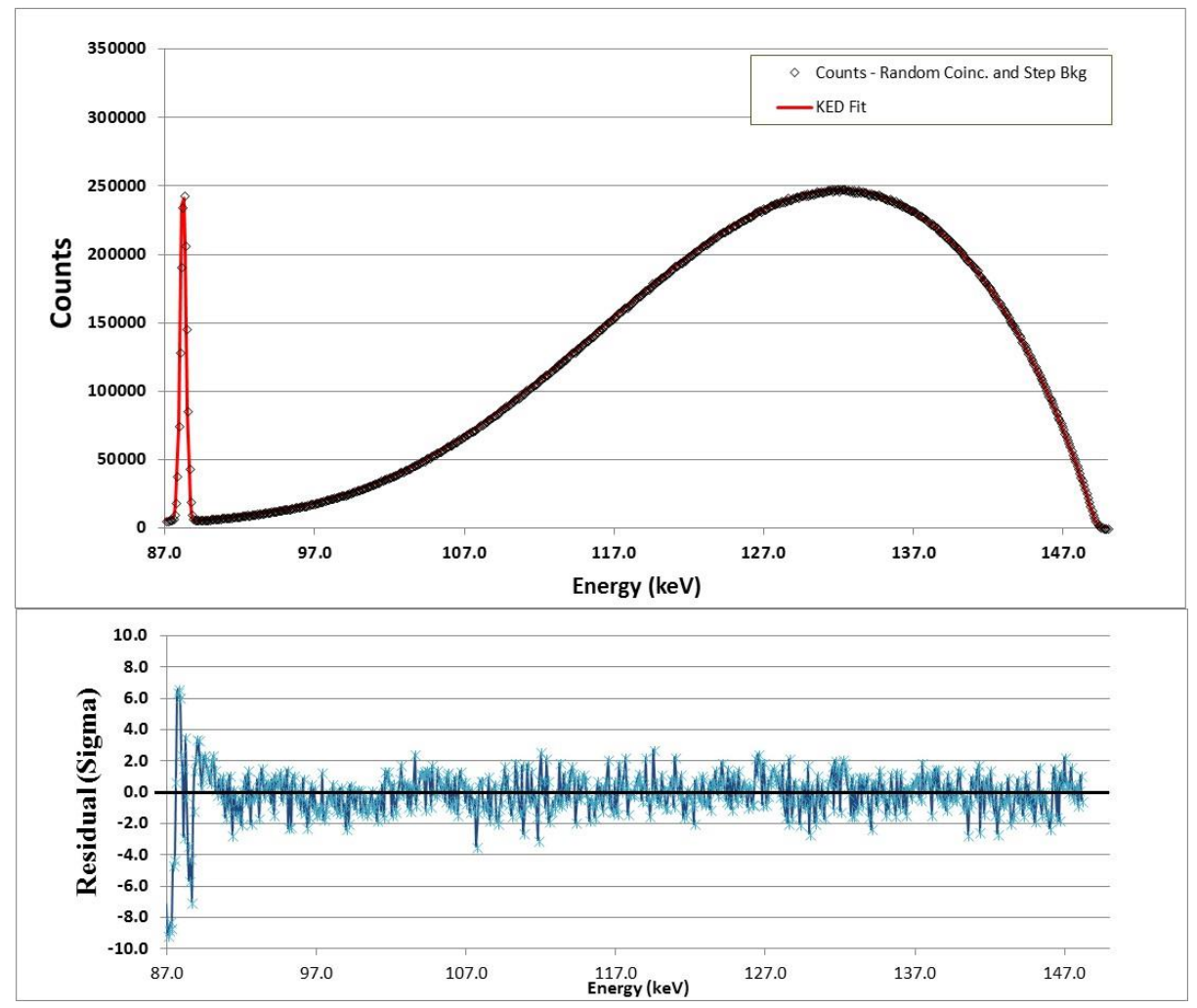

Figure 9. Fit to a KED spectrum from a $4 M$ nitric acid reference solution. 


\subsection{MASS ATTENUATION COEFFICIENTS - BIAS}

Mass attenuation coefficients used in this analysis have been derived from the XCOM database [5]. We have examined KED transmission spectra from four different HKED systems, two based on the SGN sample vial and two using the composite sample vial described in Reference [6]. In each case, we observed a consistent bias in the reported uranium and plutonium concentrations of $1.1 \%$ and $1.8 \%$, respectively. We have adjusted the mass attenuation coefficients for uranium and plutonium to provide more accurate assay. The revised mass attenuation values are provided in Appendix A.

\section{SPECTRAL FITTING MEKED PERFORMANCE}

In this section the measurement precision and accuracy of the spectral fitting approach to MEKED is presented.

\subsection{MEASUREMENT PRECISION}

The traditional KED transition analysis is performed when a single major actinide (typically uranium) is present with relatively small amounts (e.g., $\mathrm{U}: \mathrm{Pu} \geq 50$ ) of additional actinides present. The measurement performance of the traditional KED analysis was examined first by repeated measurements of the $U$ and UPu solutions standards available at ORNL, described in detail in Appendix B. These data were then reanalyzed using the spectral fitting approach. The temperature-corrected assay results for the traditional ROI analysis and the spectral fitting approach are provided in Table 5 and plotted in Figure 10. The measurement precision provided by the spectral fitting method for these runs is two to three times better than achieved by the traditional method.

Table 5. KED transmission precision estimates per assay segment (1,000 s/segment) for the ORNL standards [7]

\begin{tabular}{ccccc}
\hline ID & $\begin{array}{c}\text { Concentration } \\
(\mathrm{g} \mathrm{U} / \mathrm{L})\end{array}$ & $\begin{array}{c}\text { Assay } \\
\text { Segments }\end{array}$ & $\begin{array}{c}\text { ROI Results } \\
\text { Precision }\end{array}$ & $\begin{array}{c}\text { MEKED Results } \\
\text { Precision }\end{array}$ \\
\hline U045 & 48.12 & 9 & $0.67 \%$ & $0.23 \%$ \\
U100 & 107.09 & 15 & $0.31 \%$ & $0.15 \%$ \\
U200 & 214.45 & 9 & $0.28 \%$ & $0.11 \%$ \\
U250 & 268.40 & 18 & $0.20 \%$ & $0.10 \%$ \\
U300 & 323.69 & 15 & $0.22 \%$ & $0.07 \%$ \\
\hline UM-1 & 107.52 & 6 & - & $0.19 \%$ \\
UPu100 & 107.52 & 9 & - & $0.15 \%$ \\
UPu150 & 160.77 & 9 & - & $0.12 \%$ \\
UPu250 & 243.26 & 12 & - & $0.08 \%$ \\
\hline
\end{tabular}




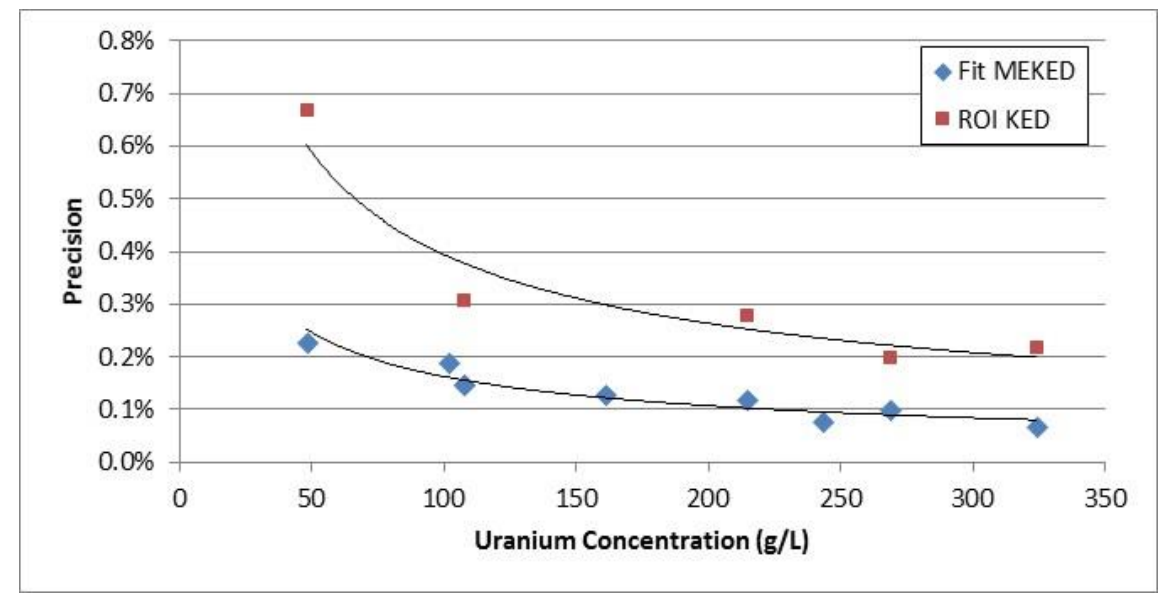

Figure 10. Measurement precision for the spectral fit MEKED analysis and the traditional ROI-based KED analysis (1,000 $\mathrm{s}$ acquisition time).

The performance of the spectral fitting method for solutions with $\mathrm{U}: \mathrm{Pu}$ ratios of approximately $1: 1$ and 2:1 was examined using a collection of 6 MOX data sets. The concentrations of these samples were determined by Isotope Dilution Mass Spectrometry (IDMS) with an assumed accuracy of $\pm 0.2 \%$. The temperature-corrected assay results using the spectral fitting MEKED are presented in Table 6 . Three of these runs (S08, S09, and S10) were replicate assays of the same solution. Since there is not a sufficiently large data set to determine the measurement precision as a function of concentration, the average measurement precision for these runs is estimated from the standard deviation of the three individual cycles comprising each run.

For a 1000 second assay time the average standard deviation for uranium was $0.15 \%$ and for plutonium $0.37 \%$ for these items.

\begin{tabular}{|c|c|c|c|c|c|c|c|c|c|c|c|}
\hline \multirow{3}{*}{$\begin{array}{l}\begin{array}{l}\text { Assay } \\
\text { Sequence }\end{array} \\
\text { S01 }\end{array}$} & \multicolumn{3}{|c|}{ Declared Values } & \multicolumn{8}{|c|}{ Spectral Fitting MEKED Analysis Results } \\
\hline & \multirow{2}{*}{$\frac{[\mathrm{U}] \mathrm{g} / \mathrm{L}}{178.37}$} & \multirow{2}{*}{$\frac{[\mathrm{Pu}] \mathrm{g} / \mathrm{L}}{82.67}$} & \multirow{2}{*}{$\frac{\mathrm{U}: \mathrm{Pu} \text { Ratio }}{2.16}$} & \multicolumn{2}{|c|}{$[\mathrm{U}] \mathrm{g} / \mathrm{L}$} & \multicolumn{2}{|c|}{$[\mathrm{Pu}] \mathrm{g} / \mathrm{L}$} & \multicolumn{2}{|c|}{$[\mathrm{Am}] \mathrm{g} / \mathrm{L}$} & \multicolumn{2}{|c|}{$\mathrm{U}: \mathrm{Pu}$ Ratio } \\
\hline & & & & 177.76 & \pm 0.12 & 82.67 & \pm 0.28 & 0.44 & \pm 0.20 & 2.150 & \pm 0.009 \\
\hline S04 & 229.07 & 105.99 & 2.16 & 228.69 & \pm 0.39 & 105.68 & \pm 0.08 & -0.12 & \pm 0.13 & 2.164 & \pm 0.003 \\
\hline S06 & 228.74 & 105.80 & 2.16 & 228.07 & \pm 0.17 & 106.07 & \pm 0.23 & 0.27 & \pm 0.16 & 2.150 & \pm 0.006 \\
\hline S07 & 118.46 & 99.40 & 1.19 & 117.34 & \pm 0.04 & 99.99 & \pm 0.33 & 1.40 & \pm 0.24 & 1.173 & \pm 0.005 \\
\hline S08 & 225.33 & 103.59 & 2.18 & 224.67 & \pm 0.41 & 103.67 & \pm 0.35 & 0.34 & \pm 0.28 & 2.167 & \pm 0.011 \\
\hline S09 & 225.33 & 103.59 & 2.18 & 225.69 & \pm 0.17 & 103.81 & \pm 0.14 & 0.62 & \pm 0.34 & 2.174 & \pm 0.002 \\
\hline S10 & 225.33 & 103.59 & 2.18 & 224.23 & \pm 0.21 & 103.49 & \pm 0.21 & 0.45 & \pm 0.22 & 2.167 & \pm 0.006 \\
\hline S11 & 171.18 & 78.71 & 2.17 & 170.55 & \pm 0.01 & 78.80 & \pm 0.06 & 0.71 & \pm 0.33 & 2.164 & \pm 0.002 \\
\hline
\end{tabular}

The measurement bias was evaluated by comparison of the assay result against the declared values and the results provided in Table 7. The average bias for uranium is somewhat larger than the assumed IDMS uncertainty and suggests a possible inaccuracy in the mass attenuation coefficients. The average bias in the plutonium concentrations agrees well with the declared values. The standard deviations for the uranium and plutonium results, both $0.3 \%$, are representative of the total measurement uncertainty for the 
$(3 \times 1,000 \mathrm{~s})$ assay. This is somewhat larger than the error reported by the KEDFIT routine, which is expected because these will include additional uncertainties such as sample positioning errors.

\begin{tabular}{|c|c|c|c|c|c|}
\hline \multicolumn{7}{|c|}{ Table 7. Assay bias resulting from the MEKED K-Edge analysis of the MOX spectra } \\
provided for this study (acquisition time 3 × 1,000 s) \\
\hline $\begin{array}{c}\text { Assay } \\
\text { Sequence }\end{array}$
\end{tabular}

The measurement bias for the ORNL solution standards are provided Table 8 for completeness. For these solutions, the uncertainties in the source declarations for uranium were much larger than the assay uncertainties. All assay results fell within $1 / 2$ sigma of the declared values, suggesting that the declared uncertainties were overstated. Although the MEKED results would not normally be used for such low plutonium concentrations, we note that the reported plutonium concentrations are in statistical agreement with the declared values.

Table 8. Comparison of the MEKED fitting results with declared values for the ORNL uranium and UPu solution standards

\begin{tabular}{cccccc}
\hline \multicolumn{2}{c}{ Declared Values } & \multicolumn{2}{c}{ Measured Values } & Bias \\
\hline $\mathrm{ID}$ & {$[\mathrm{U}](\mathrm{g} / \mathrm{L})$} & {$[\mathrm{Pu}](\mathrm{g} / \mathrm{L})$} & {$[\mathrm{U}](\mathrm{g} / \mathrm{L})$} & {$[\mathrm{Pu}](\mathrm{g} / \mathrm{L})$} & {$[\mathrm{U}]$} \\
\hline $\mathrm{U} 045$ & $48.27 \pm 0.72$ & & $48.61 \pm 0.05$ & & $0.70 \%$ \\
$\mathrm{U} 100$ & $107.30 \pm 1.49$ & & $107.73 \pm 0.09$ & & $0.40 \%$ \\
$\mathrm{U} 200$ & $214.61 \pm 2.70$ & & $214.68 \pm 0.08$ & & $0.03 \%$ \\
$\mathrm{U} 250$ & $268.21 \pm 0.21$ & & $268.28 \pm 0.08$ & & $0.03 \%$ \\
$\mathrm{U} 300$ & $321.91 \pm 4.18$ & & $319.97 \pm 0.07$ & & $0.60 \%$ \\
\hline UPu100 & $107.52 \pm 1.49$ & $1.04 \pm 0.01$ & $107.22 \pm 0.06$ & $1.31 \pm 0.09$ & $-0.28 \%$ \\
$\mathrm{UPu} 150$ & $160.77 \pm 2.04$ & $1.58 \pm 0.02$ & $160.80 \pm 0.07$ & $1.77 \pm 0.12$ & $0.02 \%$ \\
UPu250 & $243.26 \pm 2.89$ & $2.94 \pm 0.03$ & $242.59 \pm 0.13$ & $2.62 \pm 0.09$ & $-0.28 \%$ \\
\hline Average Bias & & & & $0.00 \%$ \\
Standard Deviation & & & & $0.47 \%$ \\
\hline
\end{tabular}

The capability to detect and quantify plutonium is necessary to perform the HKED's associated XRF analysis. The plutonium (and other actinide) concentrations determined from the MEKED analysis will be used to seed the Multi-Elemental XRF (MEXRF) analysis [7].

\subsection{DETECTION LEVELS AND LOW CONCENTRATION SAMPLE PERFORMANCE}

For the multi-elemental analysis, detection levels are typically only considered for the minor elements of a complex solution. For these complex solutions the detection levels for the minor actinides depend in part on both the complexity of the solution and the concentrations of the major actinide components. 
When concentrations for all actinides are low (no major actinide component) the detection levels are nominally independent of the other actinides present in the solution.

\subsubsection{Detection Levels with Uranium as the Major Actinide}

From Table 8 we can see that it is possible to detect and quantify plutonium at the gram/liter level in the presence of greater than $100 \mathrm{~g} \mathrm{U} / \mathrm{L}$. Examining the uranium-only solutions listed in the table, we can examine the detection levels for plutonium in the presence of uranium. For simplicity, we consider the detection level to be 3 times the standard deviation of the assay results for [Pu] and [Am] for 9 replicate $1,000 \mathrm{~s}$ runs. For the range of uranium concentrations examined, the detection for a single 1,000 s assay cycle is approximately $1 \mathrm{~g} \mathrm{Pu} / \mathrm{L}$ and $1 \mathrm{~g} \mathrm{Am} / \mathrm{L}$.

Table 9. Detection level for Pu in uranium solutions (measurement time 1,000 s, 3б).

\begin{tabular}{c||ccc||cc||rc}
\hline \multicolumn{1}{l||}{} & \multicolumn{3}{c||}{ Declared Values $(\mathrm{g} / \mathrm{L})$} & \multicolumn{2}{c||}{ Average Measured $(\mathrm{g} / \mathrm{L})^{*}$} & \multicolumn{2}{c}{ Detection Levels $(\mathrm{g} / \mathrm{L})$} \\
\hline ID & {$[\mathrm{U}]$} & {$[\mathrm{Pu}]$} & {$[\mathrm{Am}]$} & {$[\mathrm{Pu}]$} & {$[\mathrm{Am}]$} & {$[\mathrm{Pu}]$} & {$[\mathrm{Am}]$} \\
\hline U000 & 0 & 0 & 0 & $0.31 \pm 0.03$ & $0.31 \pm 0.04$ & 0.96 & 1.02 \\
U045 & $48.12 \pm 0.72$ & 0 & 0 & $0.43 \pm 0.10$ & $0.71 \pm 0.05$ & 0.66 & 0.51 \\
U100 & $107.09 \pm 1.49$ & 0 & 0 & $0.09 \pm 0.06$ & $0.93 \pm 0.15$ & 0.54 & 1.32 \\
U200 & $214.45 \pm 2.70$ & 0 & 0 & $0.02 \pm 0.06$ & $0.31 \pm 0.09$ & 0.54 & 0.84 \\
U250 & $268.40 \pm 0.21$ & 0 & 0 & $-0.03 \pm 004$ & $0.38 \pm 0.06$ & 0.36 & 0.84 \\
U300 & $323.69 \pm 4.18$ & 0 & 0 & $-0.01 \pm 0.06$ & $0.30 \pm 0.08$ & 0.57 & 0.75 \\
\hline Average Bias & & & 0.13 & 0.49 & & \\
Standard Deviation & & & 0.19 & 0.27 & & \\
\hline
\end{tabular}

* Average response of 9 assays of $3 \times 1000 \mathrm{~s}$ each.

An issue of concern is noted from these measurements. With no americium present, a positive bias of $0.30-0.9 \mathrm{~g} / \mathrm{L}$ for the $[\mathrm{Am}]$ result was observed. This same bias was not observable in the reported concentration for the 100:1 UPu samples listed in Table 8.

\section{CALCULATED VERSUS MEASURED REFERENCE SPECTRUM}

Determination of the X-ray source term for the KED transmission measurement during the fitting process requires implementation of a complex response function and is computationally intensive. It is therefore tempting to rely on a measured reference spectrum as is done with the classical HKED analysis. The use of a reference spectrum greatly simplifies the analysis by eliminating a number of variables such as the steel beam filter and container wall thicknesses. The expected detector response is, in principle, obtained simply by multiplication of the sample attenuation by the reference spectrum as a function of energy.

However, the KED transmission spectrum is comprised of the attenuated X-ray beam with several smaller contributions from scattering effects and response of the acquisition electronic and detector (Figure 11). Many of these components are introduced after transmission through the sample, originating within the detector or introduced by passage of the attenuated X-ray flux through the tungsten collimator. Consequently, the reference spectrum as acquired is not necessarily representative of the spectra transmitted through a sample.

Because the reference spectrum is featureless in the energy range of interest, it is difficult to deconvolve the detector response function to back out a truly representative source spectrum for analysis of a sample solution (e.g., it is not possible to determine the FWHM or tailing functions associated with the detector), so the magnitude of the effect of these response function components has not yet been evaluated. 


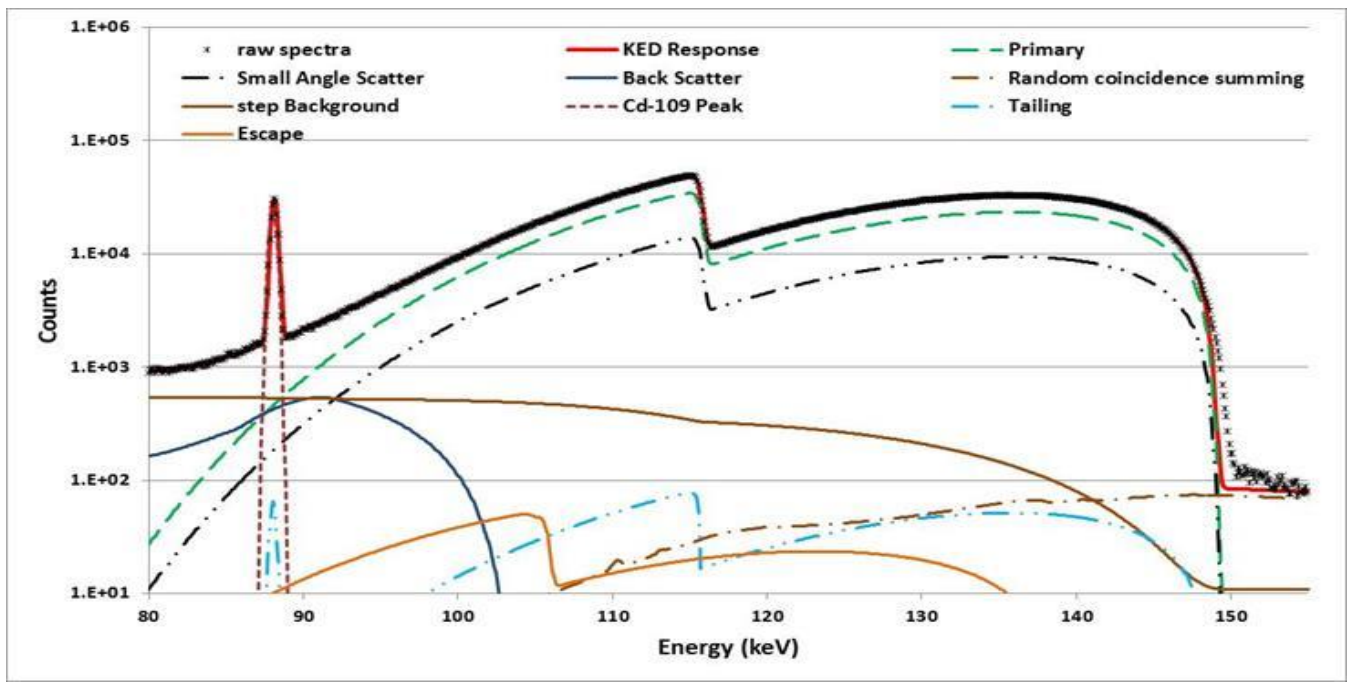

Figure 11. The fitted KED response function and various contributors to the response superimposed on a measured spectrum from a $322 \mathrm{~g}$ U/L solution standard.

\subsection{INSENSITIVITY TO X-RAY GENERATOR HV SETTINGS}

The spectral fitting approach does not require calibration as does the traditional ROI-based KED analyses, and because the method determines the X-ray source term from the observed spectrum, the method is insensitive to the HV setting. This is apparent from the plots in Figure 12 where a $75 \mathrm{~g} \mathrm{U} / \mathrm{L}$ sample was assayed at four different HV settings of the X-ray generator. In each case the measured spectrum was successfully fit, and a uranium concentration value was determined. The standard deviation of the four runs is somewhat larger than would be expected for four assays at a constant HV bias; however, there is a significant reduction in X-ray intensity with diminishing voltage. Consistent performance over a range of $30 \mathrm{kV}$ demonstrates the insensitivity of the fitting approach to the X-ray generator settings and the routine fluctuations in HV and source term.

As is illustrated in Figure 12, gain drift and shift of the X-ray endpoint energy have no impact on the algorithmic approach because the detector response function adapts to these variations as part of the fitting process. 

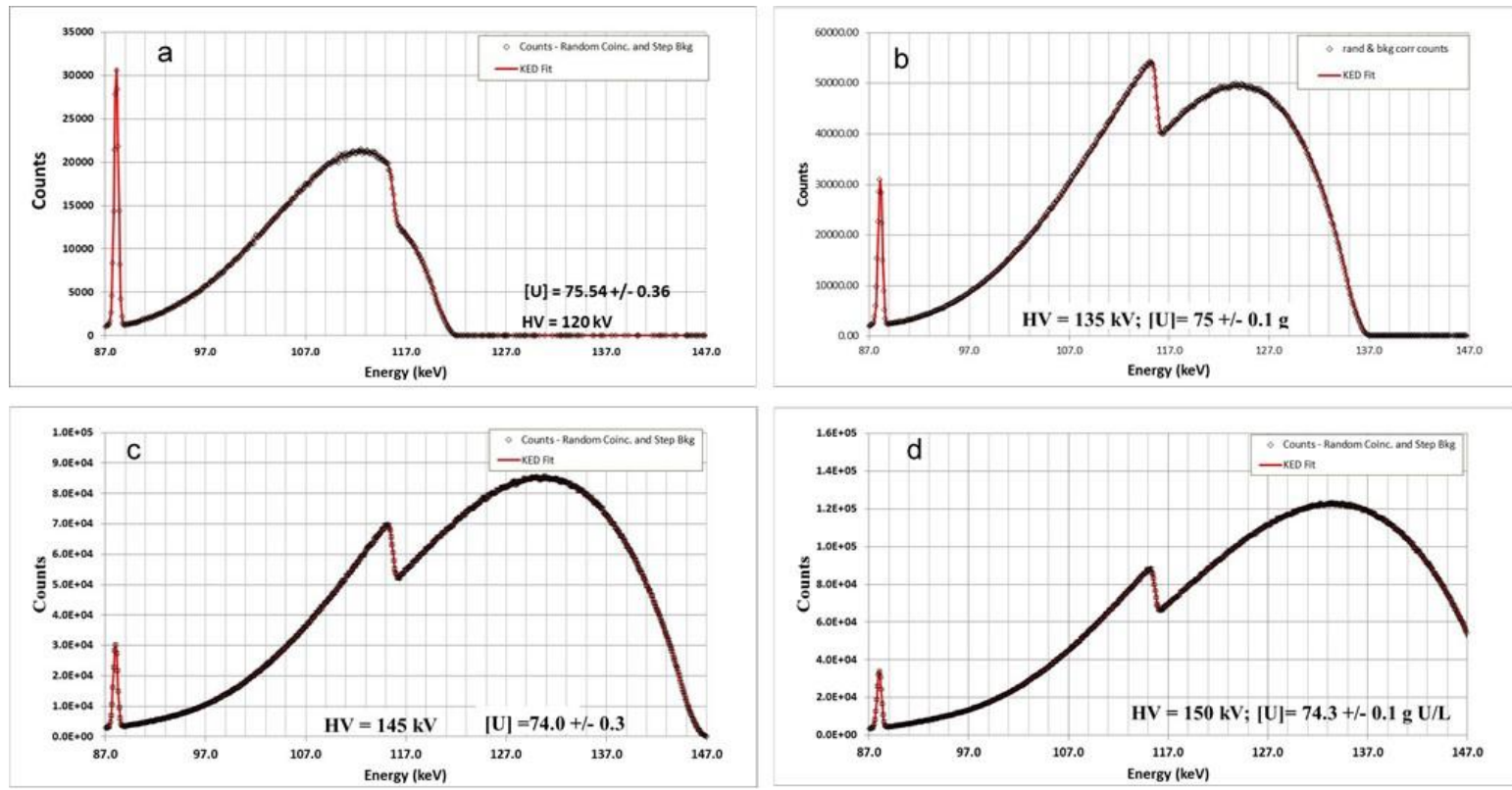

Figure 12. Plots of the KED transmission spectra with fit response for vary $\mathrm{X}$-ray generator settings (a: $120 \mathrm{kV}$, b: $135 \mathrm{kV}$, c: $145 \mathrm{kV}$, and d: $150 \mathrm{kV}$ ).

\section{KED TRANSMISSION MEASUREMENT UNCERTAINTY}

There are numerous contributors to the total measurement uncertainty of the KED transmission measurement of which the measurement precision is only one part. The following sections will provide a brief discussion of these error sources.

\section{Path Length}

Today the most commonly used samples containers for the HKED assay are polyethylene cylindrical vials with inner diameter (ID) of approximately $1.418 \mathrm{~cm}$. The ID of each vial is slightly different from the next. The path length and calculated concentration values are inversely related. A $1 \%$ overstatement of the path length results in a $1 \%$ underestimate of the concentration. The variation in the diameter from vial to vial is estimated to be $0.01 \mathrm{~mm}$ or approximately $0.07 \%$ (i.e. $0.07 \%$ in concentration).

\section{Sample Positioning}

Because the sample vial is cylindrical, a slight misalignment of the sample vial can result in a change in the path length of the K-edge transmission measurement. That is, if the sample is loaded too far or not far enough into the sample chamber, the X-ray beam will not pass through the full diameter of the vial (Figure 13). The potential impact of misalignment of the sample vial is given in Table 10. 
Table 10. Estimation of the error contribution from sample vial misalignment.

\begin{tabular}{ccc}
\hline Sample Vial & Sample Vial & Resulting Positive \\
Inner Diameter & Misalignment & Bias \\
\hline $15 \mathrm{~mm}$ & $0.5 \mathrm{~mm}$ & $0.2 \%$ \\
$15 \mathrm{~mm}$ & $1.0 \mathrm{~mm}$ & $0.9 \%$ \\
$15 \mathrm{~mm}$ & $1.5 \mathrm{~mm}$ & $2.0 \%$ \\
$20 \mathrm{~mm}$ & $0.5 \mathrm{~mm}$ & $0.1 \%$ \\
$20 \mathrm{~mm}$ & $1.0 \mathrm{~mm}$ & $0.5 \%$ \\
$20 \mathrm{~mm}$ & $1.5 \mathrm{~mm}$ & $1.1 \%$ \\
\hline
\end{tabular}

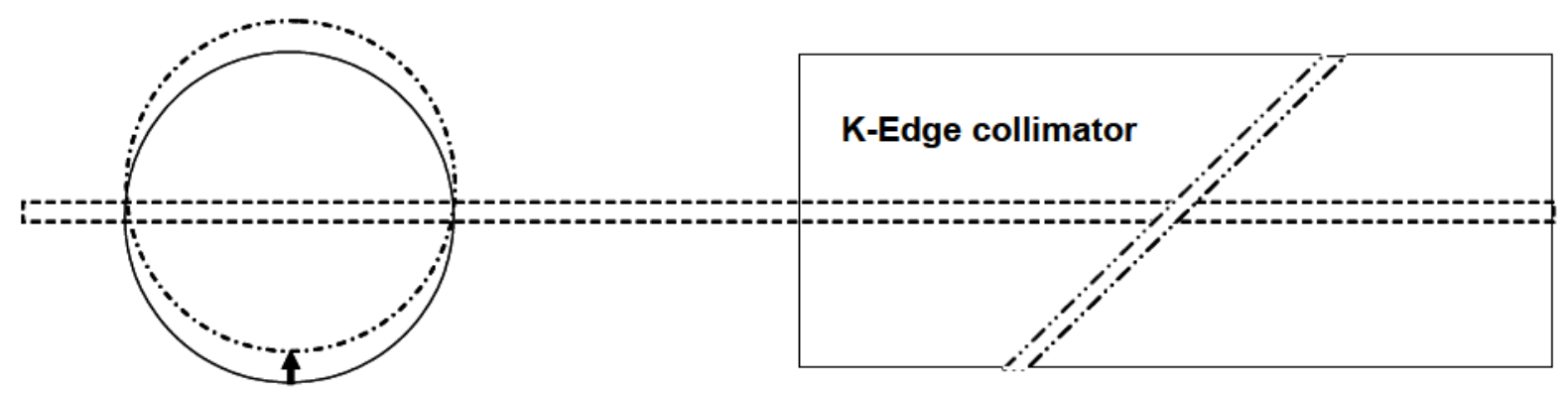

Figure 13. Illustration of the potential impact of a small shift in sample loading on the transmission measurement path length.

Sample Matrix Effects

The sample matrix (e.g., nitric acid, actinides and fission products) will have an impact on the transmission of the X-ray beam through the sample. Variations in the chemical composition of the sample matrix, if not corrected for, will introduce biases into the assay result. The traditional ROI-based analysis does not have such a correction. The error contribution to the ROI analysis has been estimated by Ottmar [1] and is expected to be generally less than $0.2 \%$ for aqueous dissolver solutions. However, the spectral fitting approach does incorporate a correction for this effect and determines the effective matrix density as part of the analysis. So this error does not contribute to the spectral fitting analysis.

\section{Uranium Enrichment}

The atomic weight of the uranium is based on a weighted average of uranium isotopic abundances within the sample. For most reprocessing materials, the uranium enrichment falls within a narrow range from 1 to $3 \%$. The variation in atomic weight is then small and should be less than $0.01 \%$ for spent light water reactor (LWR) fuels.

\section{Sample Temperature}

The sample's temperature and, to a lesser extent, the temperature of the assay system will have an impact on the KED assay result. As the temperature changes, the density of the solution changes as well as the diameter of the sample vial. We note that the $0.05 \%{ }^{\circ} \mathrm{C}$ deviation determined by Ottmar [1] is applicable to the rectangular glass cuvettes and not the polyethylene vials. The change in assay result with temperature is $+0.12 \% / /{ }^{\circ} \mathrm{C}$ for the cylindrical vial. For the spectral fitting approach, the correction is 
implemented such that (assuming the temperature reading is accurate to $0.2 \%{ }^{\circ} \mathrm{C}$ ) the error contribution to the KED analysis is $0.022 \% /{ }^{\circ} \mathrm{C}$.

\section{Calibration Constant}

Typically the error in the declarations of standards fabricated for the calibration of HKED systems is quoted as $\pm 0.3 \%$. From the assay of several standards, the calibration error for the system is reported to be approximately $0.1 \%$. However, since these standards are often fabricated by the same laboratory staff from the same source material, systematic errors can easily exceed this value. The spectral fitting approach does not require the use of standards. However, the accuracy of the atomic data tables used in the analysis is not well described. Instead we have validated the performance of the system against the analysis results from the assay of well characterized standards and have assigned a systematic error of $0.2 \%$ for the ORNL KED transmission analysis method (e.g., spectral fitting).

Non-Linearity

This error assignment encompasses electronic effects such as dead-time and tailing, which increase at high count rates. The typical error assigned is $<0.2 \%$ for use with the ROI analysis method. However, this effect is accounted for in the spectral fitting approach and so does not contribute significantly to the total uncertainty.

\section{Instrument Variability}

As with many radiation detection systems, amplifiers and other components will drift. This effect is tracked using traditional control charting approaches. Tracking of our system performance suggests that the drifting introduces less than $0.1 \%$ to the uncertainty.

\section{Summary KED Transmission Measurement Uncertainty}

The KED transmission measurement uncertainty contributors for aqueous $\mathrm{U}$ or Pu solutions are summarized in Table 11. We have followed the general format for the error analysis as presented by Ottmar et. al. [1]. Each error contribution source has been discussed above. The spectral fitting uncertainty is dominated by the uncertainty in the mass attenuation coefficients for the actinides.

Table 11. Measurement Uncertainty for the KED Transmission Measurement over the concentration range $150-300 \mathrm{~g} \mathrm{U} / \mathrm{L}$. Aqueous solution within $1.418 \mathrm{~cm}$ diameter vial.

\begin{tabular}{lcc}
\hline & \multicolumn{2}{c}{ Magnitude of the Error Component (\%) } \\
\cline { 2 - 3 } Error Contributor & ROI Approach & Spectral Fitting \\
\hline Counting Statistics (1h Assay) & 0.2 & $<0.1$ \\
Path Length (assuming each & 0.07 & 0.07 \\
Vial Diameter is measured) & & \\
Sample Positioning & $<0.1$ & $<0.1$ \\
Sample Matrix & $<0.2$ & $\mathrm{NA}$ \\
Uranium Enrichment & 0.013 & 0.013 \\
Sample Temperature & 0.06 & 0.02 \\
Calibration & 0.1 & 0.2 \\
Non Linearity & $<0.2$ & $\mathrm{NA}$ \\
Instrument Variability & $<0.3$ & $<0.1$ \\
\hline Total - Typical & 0.36 & 0.23 \\
\hline
\end{tabular}




\section{APPLICATION TO LOWER Z ELEMENTS}

The spectral fitting method is readily adapted to accommodate additional elements. Frequently discussed elements such as thorium are not expected to introduce any unique measurement problems. The addition of actinides such as thorium are simply a matter of including the relevant mass attenuation coefficients into the analysis. However, for lower Z elements, as the relevant K-Edge transition energy approaches the lower end of the transmitted X-ray spectrum (near the ${ }^{109} \mathrm{Cd}$ peak) additional measurement challenges are expected. For example, in Figure 11 above we see that the detector backscatter contribution to the KED spectrum peaks in this energy range, representing an additional interference compared to the assay of $U$ and $\mathrm{Pu}$.

As an example, an SGN vial filled with Pepto-Bismol ${ }^{\mathrm{TM}}$ was analyzed using the spectral fitting method. This common antacid contains bismuth, which has a K-Edge at $90.53 \mathrm{keV}$. The objective of this measurement was to examine the robustness of the response function in this more complicated region of the KED spectrum. Figure 14 shows a measured spectrum from the bismuth sample and provides an expanded view of the energy region about the K-Edge. Although there is excellent agreement across much of the energy range from 87 to $147 \mathrm{keV}$, the two plots show there is a statistically significant deviation between measured and fit spectra just above the K-Edge. This deviation is likely due to an inadequacy in the description of the HPGE detector backscatter response. We note that bismuth and polonium are likely the only elements to be impacted by this deviation. However, the deviation will require additional investigation before applying this method to bismuth samples.

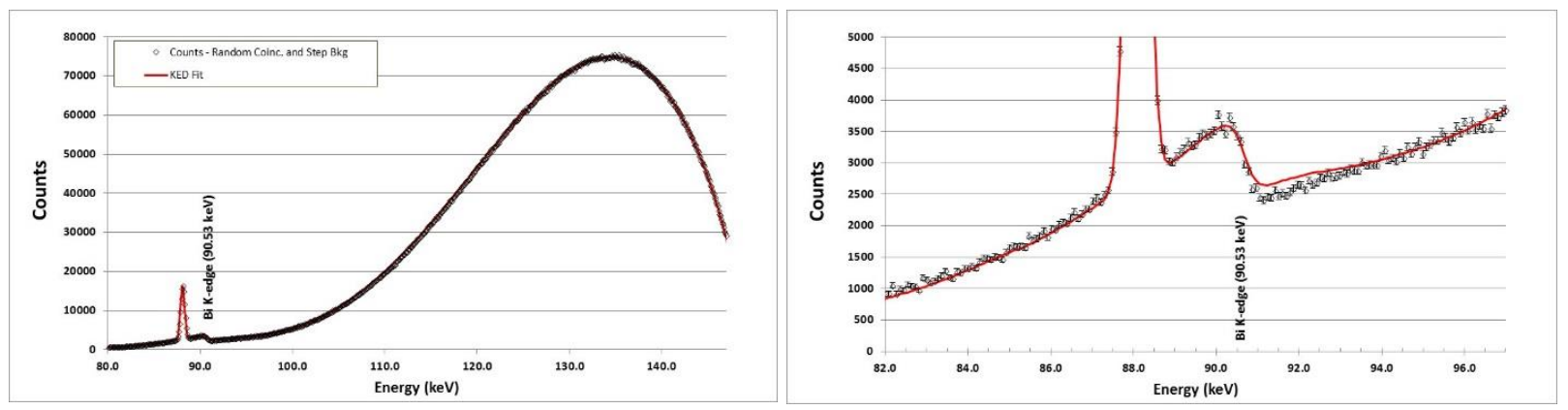

Figure 14. The measured spectra and fit response for a sample containing approximately $160 \mathrm{~g} \mathrm{Bi/L} \mathrm{(left)} \mathrm{and}$ expanded view about the Bi K-Edge (right). The deviation between the measured and expected response just above the Bi K-Edge is under investigation.

\section{CONCLUSIONS}

We have developed a multi-elemental HKED analysis method based on a spectral fitting approach. The spectral fitting approach offers improved measurement precision and accuracy and a more versatile analysis methodology than the traditional ROI-based analysis. The revised approach accommodates an arbitrary actinide mix, and currently we have incorporated five actinides into the analysis (U, Np, $\mathrm{Pu}, \mathrm{Am}$, and $\mathrm{Cm}$ ); however, it is a simple matter to incorporate additional isotopes. Measurement precision is improved by a factor of 2 or more by using the spectral fitting approach for both the MEKED (and MEXRF analyses [8]). The analysis is better able to accommodate instrumental variation such as detector gain and drifting of the X-ray generator HV supply.

The MEKED transmission measurement is essentially calibration free, however, performance can be further improved by full characterization of the assay system (i.e., determination of collimator/attenuator thickness). Accuracy of the KED transmission measurement is limited by the accuracy of the XCOM database [5], although assay results are consistent with declaration values. Based on analysis of ORNL 
and International Atomic Energy Agency HKED spectra, MEKED analysis biases for uranium and plutonium solutions are no more than $\pm 0.3 \%$. Unfortunately, the available calibration standards are not sufficiently well known to fully characterize the KED performance. This could be remedied by compiling the calibration results from multiple HKED systems. Because the HKED systems are generally calibrated using working standards fabricated at the end-use facility, systematic errors could be eliminated by combining these results to generate revised cross-section tables over the energy range of interest to the HKED.

Development Needs

- Testing with Dissolver Solutions. Historically, it has been assumed that the impact of the fission product decay on the HKED response is small. This assumption needs to be verified both for the MEKED algorithms and for more complex dissolver solutions resulting from today's fuel cycle. To date there has been no significant testing of the ORNL MEKED analysis with fission product loaded solutions.

- The spectral fitting MEKED analysis is currently implemented in Visual Basic and with Microsoft Excel serving as the user interface. The fitting routines have not been developed under the guidance of a formal quality assurance program. It will be necessary to have the spectral fitting MEKED analysis properly coded in a modern language such as $\mathrm{C}++$ under a formal quality assurance/quality control program before fielding the analysis on other than an investigational level.

- The photoelectric component of the mass attenuation coefficients listed in the XCOM database used in this analysis, are not based on measured values but are calculated with only a cursory error analysis. The accuracy of these coefficients ultimately limits the performance of the spectral fitting MEKED. The attenuation coefficients could be verified by direct measurement, such as attempted by Materna et al. [9], or by analysis of a collection of HKED spectra from different facilities.

- We have noted a persistent positive bias in the reported americium concentrations for solutions containing only uranium. The origin of this bias needs to be identified and corrected. This bias is not present for any of the other actinides examined in this study.

- The reported uncertainties from the KEDFIT routine sometimes appear somewhat optimistic (too small) relative to the observed measurement precision. This is in part due to uncertainties not included in the analysis (e.g., sample positioning errors) but also to the nature of the complexity of the fitting process. A typical analysis addresses 13-19 free parameters, so there is a strong likelihood that local minima will be encountered during the $\chi^{2}$ minimization process. This has been addressed by relaunching the fitting process a second time using a slight perturbation of the initial fit results. We then choose the result providing the smaller $\chi^{2}$ value. Although this has been effective, it is by no means an efficient or robust methodology. A more rigorous method for avoidance of local minima during the fitting process is required.

- Better standards are needed. Historically, the HKED systems have been calibrated using working reference solutions created from either process samples or stock solutions [10]. These working standards are characterized via Isotope Mass Dilution Spectrometry (IDMS) or similar technique. However, over the primary concentration range (100 to $300 \mathrm{~g} \mathrm{U} / \mathrm{L}$ ) targeted by the HKED measurement, the HKED assay yields measurement precision superior to that of the IDMS result. The true performance of the HKED analysis can not be fully evaluated because suitable standards are not available. 
- X-ray End-point Energy disparity. The source of the high energy tailing of the generator's X-ray distribution needs to be established. The MEKED analysis is intended to rely as much as possible on first principles to minimize potential uncertainties and reduce the need for standards. To completely accomplish this the measurement response should be fully understood.

- Acquisition Electronics

- The pile-up rejection capabilities of the acquisition electronics (both ICB and Lynx) are not completely effective. The limitations of the pile-up rejection need to be explored.

- The live time correction of the Lynx digital signal processors on the ORNL system, appears to be ineffective. We have noted significant variation $(>10 \%)$ in the ${ }^{109} \mathrm{Cd}$ count rate with changes in the X-ray generator beam current (e.g. $5 \mathrm{~mA}$ vs $15 \mathrm{~mA}$ ). While the impact to the KED and hybrid analyses are negligible, the stand-alone XRF measurement has become unusable. The cause of this behavior needs to be determined.

- $\quad{ }^{109} \mathrm{Cd}$ reference source. We have demonstrated that the ${ }^{109} \mathrm{Cd}$ gamma-rays used for gain stabilization are a potential interference for the assay of solutions containing bismuth. An alternative gain stabilization method will be required in order to adapt the HKED to pyroprocessing facilities. 


\section{REFERENCES}

[1] H. Ottmar and H. Eberle, "The Hybrid K-Edge/K-XRF Densitometer: Principles - Design Performance; Report KfK 4590," Karlsruhe, 1991.

[2] International Standards Organization;, Simultaneous determination of uranium and plutonium in dissolver solutions from reprocessing plants--Combined method using $K$-absorption edge and $X$-ray fluorescence spectrometry, 1998.

[3] H. Zhu, J. Lamontange, S. Croft, H. Hassoubi, G. Landry, R. D. McElroy, Jr., R. Patel, S. Philips, P. Rouleau, R. Venkataraman and V. Yuschuk, "A New Software Application for Hybrid Densitometry," in Proceedings of the 50th Annual Meeting of the Institute of Nuclear Materials Management (INMM), July 12-16, Tucson, Az, 2009.

[4] P. R. Bevington, Data Reduction and Error Analysis for the Physical Sciences, New York: McGraw-Hill, 1969.

[5] M. J. Berger, J. H. Hubbell, S. M. Seltzer, J. S. Coursey, R. Sukumar, D. S. Zuker and K. Olsen, "XCOM: Photon Cross Sections Database," 1998. [Online]. Available: http://www.nist.gov/pml/data/xcom/index.cfm. [Accessed 2015].

[6] R. D. McElroy, "Performance Evaluation of the CHKED Multi-Elemental Analysis Software, Technical Report ORNL/TM-2016/257," Oak Ridge National Laboratory, Oak Ridge, TN, 2016.

[7] R. D. McElroy, S. Croft, G. S. Mickum and S. L. Cleveland, "Spectral Fitting Approach to the Hybrid K-Edge Densitometer: Preliminary Performance Results," in 56th Annual Meeting of the Institute of Nuclear Materials Management (INMM 56) (Proc. Conf. Indian Wells, CA, 2015), Curran Associates, Inc., Indian Wells, CA, 2015.

[8] R. D. McElroy, "Performance Evaluation of the ORNL Multi-Elemental XRF (MEXRF) Analysis Algorithms, Report No ORNL/TM-2016/594," Oak Ridge National Laboratory, Oak Ridge, TN, 2016.

[9] T. Materna, J. Jolie, W. Mondelaers and B. Masschaele, "Near K-edge measurement of the X-ray attenuation coefficient of heavy elements using a tuneable X-ray source based on an electron LINAC," Radiation Physics and Chemistry, vol. 59, pp. 449 - 457, 2000.

[10] T. F. Guzzardo, R. D. McElroy, Jr., S. Croft, J. Garrison, R. Venkataraman and C. A. Pickett, "Stability of Working Reference Standards for Hybrid K-edge Densitometer Quality Assurance, IAEA-CN-220(2014)-284," in Symposium on Internation Safeguards, Linking Strategy, Implementation and People,., Vienna, Austria, 2014.

[11] A. A. Shaltout, "On the X-Ray Tube spectra, the dependence on the angular and electron energy of X-rays from the targets," Eur. Phys. J. Appl. Phys., vol. 37, pp. 291-297, 2007.

[12] R. D. McElroy, S. L. Cleveland, S. Croft, G. S. Mickum and A. Nicholson, "Relative Actinide KShell Vacancy Production Rates in Hybrid K-Edge Densitometry," in 37th ESARDA symposium on Safeguards and Nuclear Non-Proliferation, Manchester, UK, 19-21 May 2015.

[13] J. T. Goorley and et.al., "Initial MCNP6 Release Overview - MCNP6 version 1.0, LA-UR-1322934," Los Alamos National Laboratory, Los Alamos, NM, 2013.

[14] B. K. Agarwal, X-Ray Spectroscopy: An Introduction, Springer-Verlag Berlin Heidelberg GmbH, 1991.

[15] R. D. McElroy, Jr., S. Croft, S. L. Cleveland and G. S. Mickum, "Spectral Fitting Approach to the Hybrid K-Edge Densitometer, Preliminary Performance Results," in Proceedings of the INMM 56th Annual Meeting, Indian Wells, CA., USA, 2015. 
[16] S. Croft, R. D. McElroy, Jr. and T. Guzardo, "Representing the Plutonium K-absorbtion Edge in Transmission Measurements," in Proceeding of the 55th Annual Meeting of the Institute of Nuclear Materials Management (INMM) July 20-24, Atlanta, GA, 2014.

[17] R. Gunnink, "An Algorithm for Fitting Lorentzian-Broadened, K-Series X-ray Peaks of the Heavy Elements," Nucl. Instr. and Meth., vol. 143, pp. 145-149, 1977.

[18] R. Gunnick and J. B. Niday, "Precise Analyses by Gamma-Ray Spectroscopy," in ACS Annual Meeting, August 24-29 1975, Chicago, 1975.

[19] G. F. Knoll, Radiation Detection and Measurement, New York: John Wiley and Sons, 1979.

[20] J. S. Hendricks and et.al., "MCNPX Version 2.5, LA-UR-02-7086," Los Alamos National Laboratory, Los Alamos, NM, 2002.

[21] R. Venkataraman and et.al., "Improved Detector Response Characterization Method in ISOCS and LabSOCS," in Methods and Applications of Radioanalytical Chemistry (MARC VI), Kailua-Kona, HI, 2003.

[22] E. Browne and R. B. Firestone, Table of Radioactive Isotopes, New York: John Wiley \& Sons, 1986, pp. C-15.

[23] J. L. Campbell and T. Papp, "Widths of the Atomic K-N7 LEVELS," ADNDT, vol. 77, no. 1, pp. 156, 2001.

[24] F. K. Richtmyer, S. W. Barnes and E. Remberg, "The Widths of the L-Series and of the Energy Levels of Au (79)," Phys. Rev., vol. 46, p. 834, 1934. 


\section{APPENDIX A. MEKED SPECTRAL RESPONSE REPRESENTATION}

We have created a spectral fitting methodology for analysis of the K-Edge densitometry (KED) transmission measurement. This approach takes full advantage of the information content of the transmission spectra allowing determination of the major actinide and matrix concentration for the sample solution. The K-Edge transmission measurement is effectively a first principles measurement and should therefore require no calibration other than the energy calibration of the HPGe detector. However, the KED spectrum is remarkably complex due to scattering and electronic effects (Figure A1). To reduce the complexity of the analysis, we limit the fitting function to encompass the energy range spanning the ${ }^{109} \mathrm{Cd}$ peak at $88 \mathrm{keV}$ to just below the X-ray generator endpoint energy (i.e., 85-147.5 keV). Even with this limitation, the list of potential free parameters is rather lengthy as summarized in Table A1 and illustrated in Figure A1.

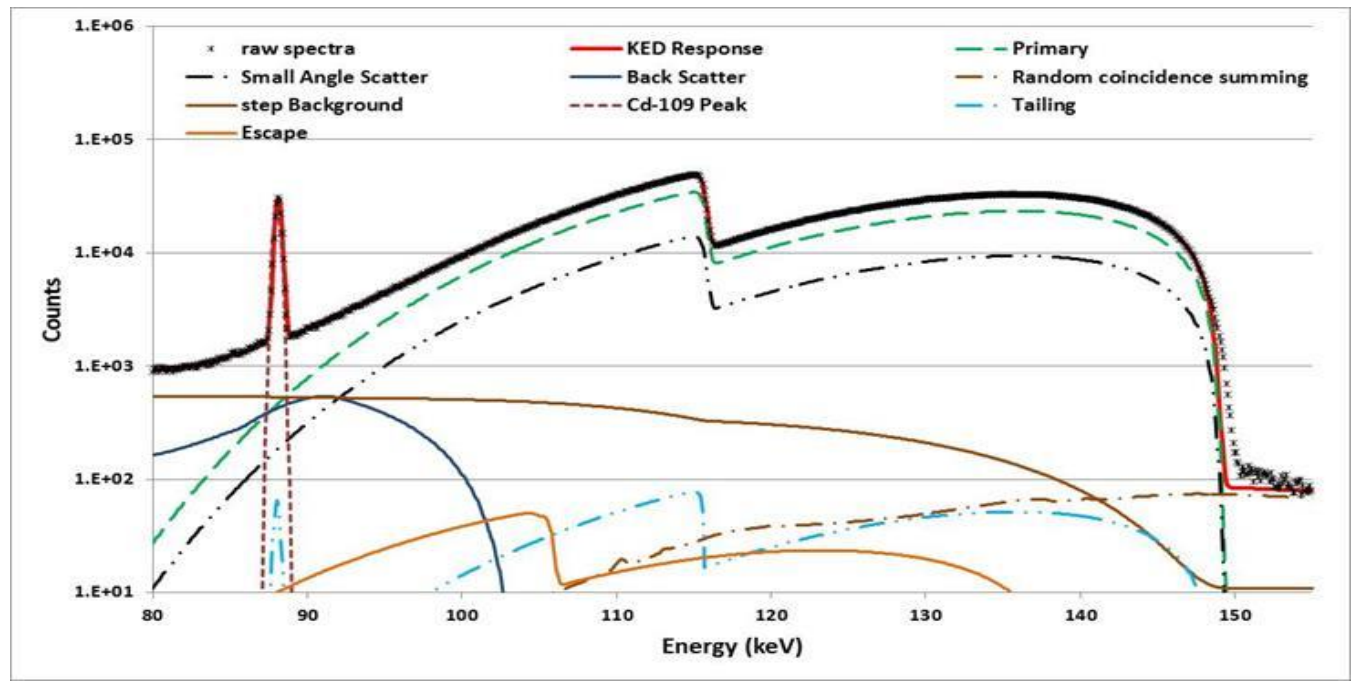

Figure A1. The fitted KED response function and various contributors to the response superimposed on a measured spectrum from a $322 \mathrm{~g}$ U/L solution standard. The deviation near the $\mathrm{HV}$ cutoff is tentatively attributed to jitter in the HV supply in the X-ray generator.

Table A1. Fit parameters for the KED transmission measurement

\begin{tabular}{lll}
\hline Sample parameters & Detector parameters & X-ray generator parameters \\
\hline U Concentration & Energy Offset & Endpoint Energy \\
Np Concentration & Energy Slope & X-ray Intensity \\
Pu Concentration & Gaussian Width & Shape Parameter [11, 12] \\
Am Concentration & Fast Tail Decay & Small Angle Scatter Fraction \\
Cm Concentration & Fast Tail Intensity & Detector Backscatter Intensity \\
Matrix concentration & Slow Tail Decay & \\
& Slow Tail Intensity & \\
\hline
\end{tabular}

Our KED response function uses Shaltout's representation [11] of the X-ray source term modified by the assembly of attenuating layers (e.g., beam filters, sample vial, detector housing), the sample contents (i.e., the actinides and sample matrix), and detector response function. Examination of experimental KED spectra supplemented by Monte Carlo N-Particle (MCNP) [13] modeling showed that the transmitted spectrum includes a substantial contribution from small angle scattering within the long, narrow tungsten 
collimator $(120 \times 0.8 \mathrm{~mm})$, as well as a small backscatter peak from the detector's copper cold finger assembly.

In our representation of the KED response function, we incorporate the mass attenuation data from the National Institute of Standards and Technology XCOM Photon Cross Sections Database [5] and assume for the present that these values have no uncertainty. These cross-section tables provide a delta function step at the K-Edge transition, whereas others assume that the K-Edge transition is Lorentzian broadened [9] [14] [15] [16]. We have found that a mixture of the two assumptions provides the best empirical representation for the KED response function. That is, for energies below the K-Edge transition, a Voigt broadening of the mass attenuation function is required, but above the K-Edge, a simple Gaussian broadening provides the best representation. The shape of high-energy response is potentially explained as an artifact of the fine structure in the mass attenuation function above the edge that is smoothed out by the instrumental response; however, more detailed cross-section data will be needed to verify this conjecture. The resulting response function provides an excellent representation of the observed data as illustrated in Figure A2.

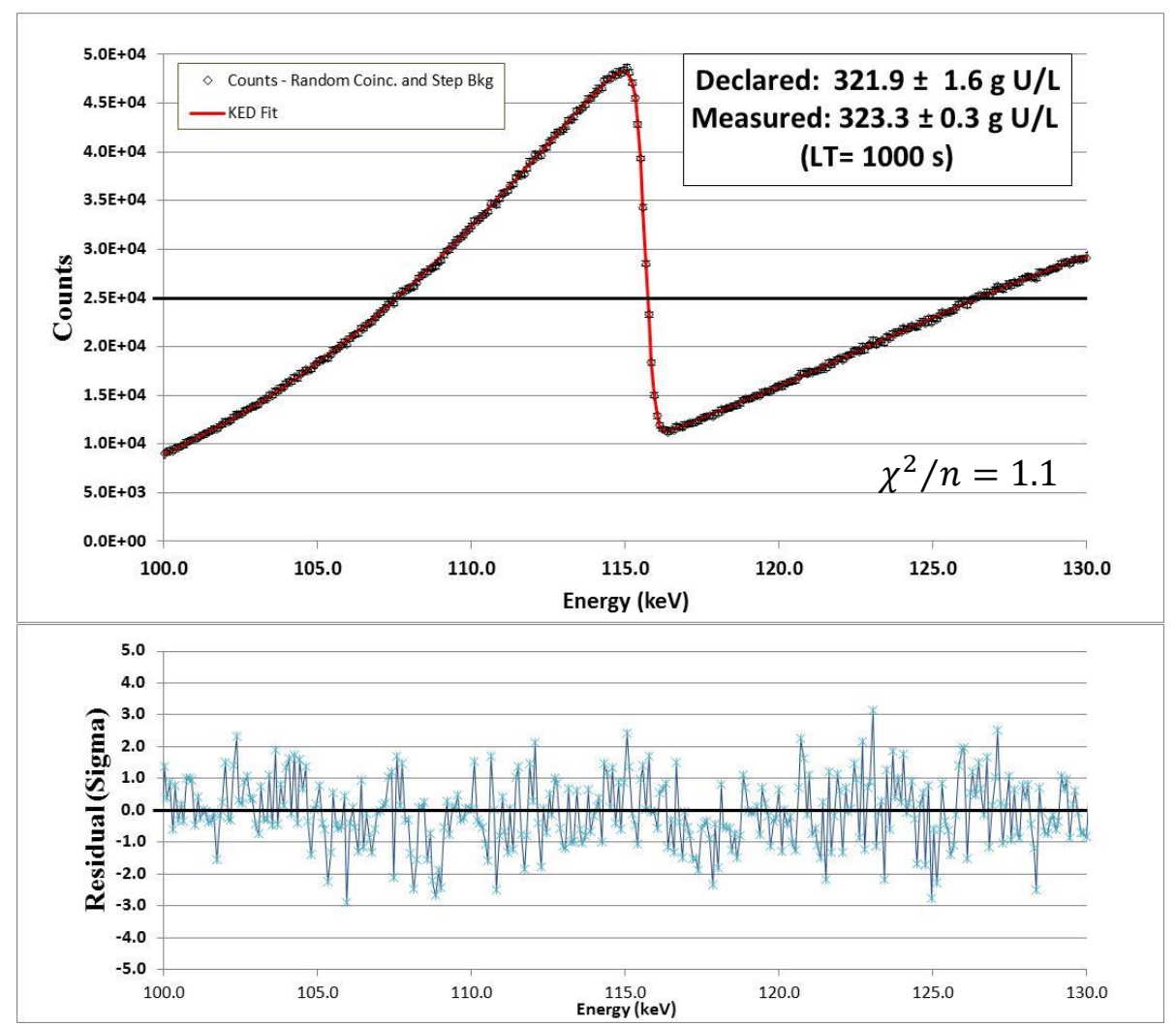

Figure A2. KED spectrum obtained from a solution containing approximately

$322 \mathrm{~g} \mathrm{U} / \mathbf{L}$. The upper plot shows the fit to the measured spectrum after correction for background and random coincidence summing. The lower plot shows the relative residuals in terms of multiple standard deviations and illustrates the fidelity of the

KED fitting process.

\section{A-1. KED RESPONSE FUNCTION}

The KED response function is discussed relative to the source term, $f_{K E D}$, and the detector response function, $f_{\text {det }}$. The source term includes the Bremsstrahlung distribution from the X-ray tube and two 
scattering components that are related to the Bremsstrahlung source term and subject to attenuation by both the sample and the system hardware. The source term also includes the ${ }^{109} \mathrm{Cd}$ gamma-ray peak at $88.034 \mathrm{keV}$ (included to provide a reliable energy scaling for low concentration samples). The response function can be written as

$$
f_{K E D}(E)=\left[f_{\text {Brem }}(E)+f_{\text {small }}(E)+f_{\text {back }}(E)\right] \cdot \delta_{\text {attn }}(E) \cdot \delta_{\text {samp }}(E)+I_{C d}
$$

where the detector response function, $f_{\text {det }}\left(E, E^{\prime}\right)$, is given as a function of energy, $E^{\prime}$, and $E$ is the incident photon energy,

$$
f_{\text {det }}\left(E, E^{\prime}\right)=\varepsilon(E) \cdot\left(g\left(E, E^{\prime}\right)+f_{\text {tail }}\left(E, E^{\prime}\right)+f_{\text {escape }}\left(E, E^{\prime}\right)+b_{\text {step }}\left(\mathrm{E}, \mathrm{E}^{\prime}\right)\right) .
$$

The KED spectrum, $C_{K E D}$, is represented by the convolution of the detector response function and the source term, $f_{K E D}$, with the addition of the random coincidence summing, $f_{r_{s u m}}$, and general background, $f_{b k g}$, contributions

$$
C_{K E D}(E)=\left(f_{\text {det }} * f_{K E D}\right)(E)+f_{r_{s u m}}(\mathrm{E})+f_{b k g}(\mathrm{E}),
$$

where $f_{\text {Brem }}(E)$ is Bremsstrahlung radiation source term as a function of energy for photons emitted from the X-ray tube's tungsten target;

$f_{\text {small }}(E)$ is the small angle scattering component;

$f_{\text {back }}(E)$ is backscatter component;

$\delta_{a t t n}(E)$ represents the attenuation of the emitted Bremsstrahlung radiation source term by the various filters, foils, system housing and sample vial wall thickness;

$\delta_{\text {samp }}(E)$ represents the attenuation of the emitted Bremsstrahlung radiation source term by the sample (i.e., nitric acid matrix, and actinides);

$g\left(E, E^{\prime}\right)$ is the Gaussian distribution as a function of $E^{\prime}$ and centered at energy $E$;

$\varepsilon(E)$ is the relative detection efficiency;

$f_{\text {escape }}(E)$ is the contribution to the spectrum due to the germanium "escape peaks";

$f_{\text {step }}(E)$ is the step background associated with each X-ray fluorescence (XRF) peak;

$f_{\text {tail }}(E)$ is HPGe detector tailing function;

$I_{C d}(E)$ is the ${ }^{109} \mathrm{Cd}$ peak intensity from the $88 \mathrm{keV}$ line;

$f_{r_{-} s u m}(E)$ is contribution from random coincidence summing;

$f_{b k g}(E)$ is an additional polynomial background function allowed in the fitting routine (this background function is presently not used).

Each of the components of the KED response function will be discussed in the following sections of this report. Note that in this study we consider as source terms any photons originating outside of the HPGe detector crystal including the detector backscatter contribution, which effectively originates in the copper cold finger behind the detector crystal. 


\section{A-2. SOURCE TERMS}

\section{X-ray Source Term: $f_{X}(E)$}

There are many representations of the X-ray source term described in the literature; however, we have chosen to use that of Shaltout [11] because of the completeness of their description. The interrogating Xray source term is given by

$$
f_{\text {Brem }}(E)=C \cdot \Omega \cdot I \cdot Z \cdot f_{a}(E) \cdot\left(\frac{E_{0}}{E}-1\right)^{x},
$$

where $f_{a}(E)=\frac{\left(1-e^{-2 \cdot \mu_{W}(E) \cdot \overline{\rho z} \cdot \sin (\varphi) / \sin (\epsilon)}\right)}{2 \cdot \mu_{W}(E) \cdot \overline{\rho z} \cdot \sin (\varphi) / \sin (\epsilon)}$

$$
\begin{aligned}
& \overline{\rho Z}=\rho z_{m} \cdot \frac{\left(0.49269-1.0987 \cdot \eta+0.78557 \cdot \eta^{2}\right)}{\left(0.70256-1.09865 \cdot \eta+1.0046 \cdot \eta^{2}+\ln \left(U_{0}\right)\right)} \cdot \ln \left(U_{0}\right), \\
& \rho z_{m}=\frac{A}{Z} \cdot\left(0.787 \cdot 10^{-5} \cdot \sqrt{J} \cdot E_{0}^{\frac{3}{2}}+0.735 \cdot 10^{-6} \cdot E_{0}^{2}\right), \\
& \eta=E_{0}^{m} \cdot\left(0.1904-0.2236 \cdot \ln (Z)+0.1292 \cdot \ln (Z)^{2}-0.0149 \cdot \ln (Z)^{3}\right),
\end{aligned}
$$

and

$$
U_{0}=\frac{E_{0}}{E},
$$

$C$ is a constant,

$I$ is the X-ray tube beam current,

$\Omega$ is the solid angle of the detector (or in our case the interaction region),

$Z$ is the atomic number of the $\mathrm{X}$-ray tube tungsten target (i.e., 74),

$A$ is the atomic weight of the $\mathrm{X}$-ray tube tungsten target (i.e., 183.84),

$\mathrm{x}$ is empirically determined to match the observed X-ray energy distribution and is $\sim 0.92$ for our system

$\varphi$ is the angle of incidence for $\mathrm{e}^{-}$beam in the $\mathrm{X}$-ray tube

$\epsilon$ is the takeoff angle for the X-rays emitted from the X-ray tube.

$\mu_{W}(E)$ is the mass attenuation coefficient of tungsten,

$E_{0}$ is the X-ray tube endpoint energy,

$m=0.1382-0.9211 / \sqrt{Z}=0.03112$,

$J=0.135 \cdot Z=9.99$ for the $\mathrm{W}$ target.

\section{Attenuation due to System Hardware}

We distinguish between attenuators based on variability, the hardware filters are fixed for a given system while sample parameters are generally unknown and variable. The following fixed attenuators are present between the X-ray source and the HPGe detector:

- Beryllium window, X-ray tube exit window-Approximately $0.3 \mathrm{~mm}$ thick Be.

- Cd beam filter - $1 \mathrm{~mm}$ thick cadmium foil located between the transfer tube beam entrance wall and the X-ray tube. 
- Transfer tube beam entrance wall-Approximately 0.4 to $2.6 \mathrm{~mm}$ thick stainless steel.

- Transfer tube beam exit wall-Approximately 0.4 to $2.6 \mathrm{~mm}$ thick stainless steel.

- Steel beam filter-Approximately $24 \mathrm{~mm}$ thick mild steel.

- Sample vial—For the SGN-style sample vial, the total thickness of HDPE is $\sim 2 \mathrm{~mm}$.

$$
\delta_{a t t n}(E)=e^{-\sum \mu_{a_{i} \cdot} \cdot \rho_{a_{i}} \cdot t a_{i}},
$$

where $\mu_{a_{i}}$ is the mass attenuation coefficient for absorber $a_{i}$,

$\rho_{a_{i}}$ is the density for absorber $a_{i}$,

$t_{a_{i}}$ is thickness of absorber $a_{i}$,

An illustration of the placement of these attenuators in the system is shown in Figure A3.

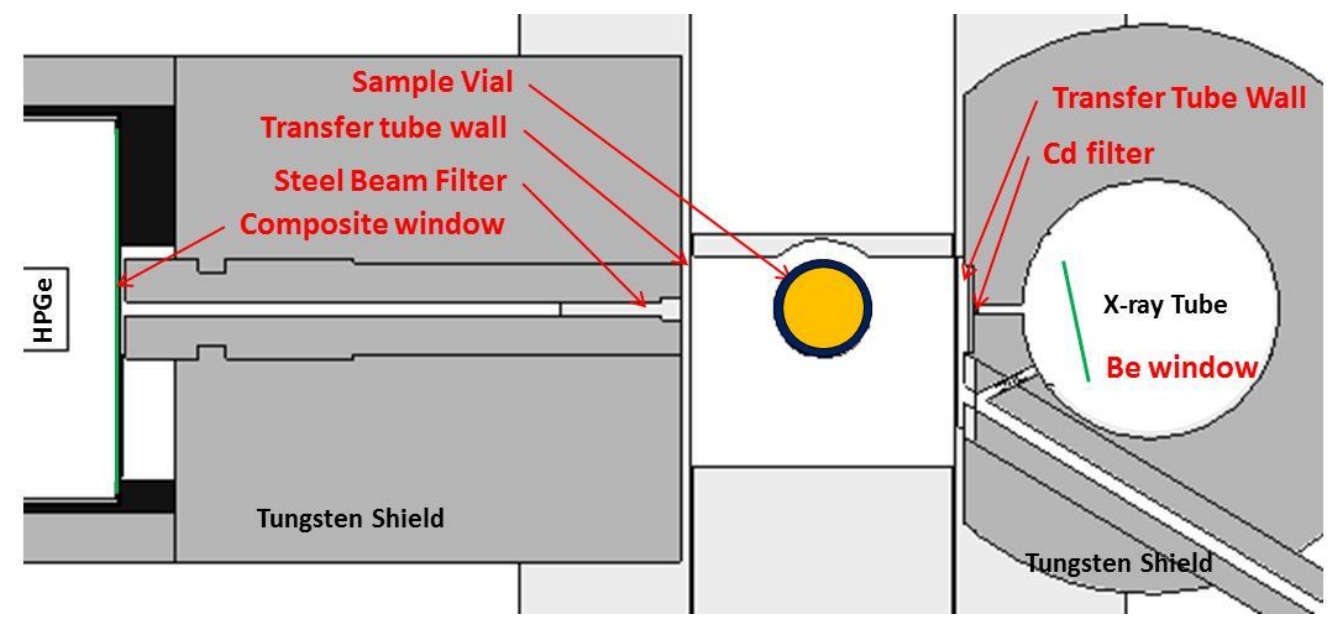

Figure A3. Diagram of the KED transmission sub-system attenuators.

\section{Sample Attenuation}

Attenuation due to the sample constituents is treated separately from fixed attenuators because they will vary from sample to sample. The sample matrix (e.g., nitric acid or salt) is a composite material and each component must also be included in the attenuation factor

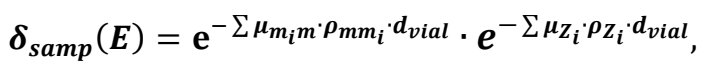

where $\mu_{Z_{i}}$ is the mass attenuation coefficient for the $\mathrm{i}^{\text {th }}$ actinide $Z_{i}$,

$\rho_{Z_{i}}$ is the partial density of the $\mathrm{i}^{\text {th }}$ actinide $Z_{i}$,

$\mu_{m_{i}}$ is the mass attenuation coefficient for the solution matrix,

$\rho_{m_{i}}$ is the partial density for the solution matrix,

$d_{\text {vial }}$ is the inner diameter of the sample vial. 


\section{Small Angle Scattering Component}

Small angle scattering was introduced into the KED transmission analysis in an attempt to better describe the spectral behavior near the K-Edge transitions. The $0.8 \mathrm{~mm}$ diameter aperture through the tungsten collimator limits the photon intensity incident on the HPGe detector, ensures that the incident photons are nearly normal to the surface of the detector, and confines detection to a relatively small area of the detector surface. All of these attributes serve to provide a clean detector response, limit dead-time, and provide the highest resolution possible. However, small angle scattering of X-rays within the collimator produces a secondary spectrum with a low energy offset relative to the non-scattered X-rays. MCNP modeling of the HKED system indicated that approximately one-fourth of the X-rays reaching the detector have undergone small angle scattering.

There are two approaches to correct for the small angle scattering component. The first is to develop an analytical expression beginning with the attenuated X-ray fluence described by Eq. (4), calculating the scattering fraction using the Klein-Nishina formalism, giving a response function

$$
f_{\text {small }}(E)=f_{\text {scat }} \cdot \varepsilon(E) \cdot f_{\text {Brem }}\left(E^{\prime}\right) \cdot \delta_{\text {attn }}\left(E^{\prime}\right) \cdot \delta_{\text {samp }}\left(E^{\prime}\right) \text {, }
$$

where $E$ is the energy of the scattered photon,

$\mathrm{E}^{\prime}$ is the photon energy before scattering (i.e., the incident energy),

$E^{\prime}=E /\left(1-\alpha_{1} \cdot(1-\cos (\theta))\right)$

$m_{e}$ is the rest mass of the electron in $\mathrm{keV}$,

$\alpha_{1}=E / m_{e}$,

$\theta$ is the angle in radians of the KED tungsten collimator (for our system $\theta=0.008$ ) and

$f_{\text {scat }}$ is an empirically determined weighting factor.

Note that given the very narrow KED collimator, the energy shift due to the small angle scattering is approximately less than $0.001 \mathrm{keV}$ at $100 \mathrm{keV}$. Given the resolution of the HPGe detection system, it is not possible to distinguish the scattered spectrum from the unscattered one. The primary impact of the small angle scattering is to slightly broaden the spectral resolution; however, its inclusion in the spectral response is necessary to accurately reproduce the observed spectra. Figure A4 illustrates the relative intensities of the scattered and unscattered components of the spectrum.

The second method is simpler but not robust and has not been thoroughly tested. In this method, the observed spectrum is scaled in amplitude and slightly offset in energy. The scaled spectrum is then subtracted from the original spectrum. During data analysis, the scaling factor and energy offset would be varied during the fitting process. This method appears to represent the scattered spectrum well, but we have only examined the performance over a limited energy range. 


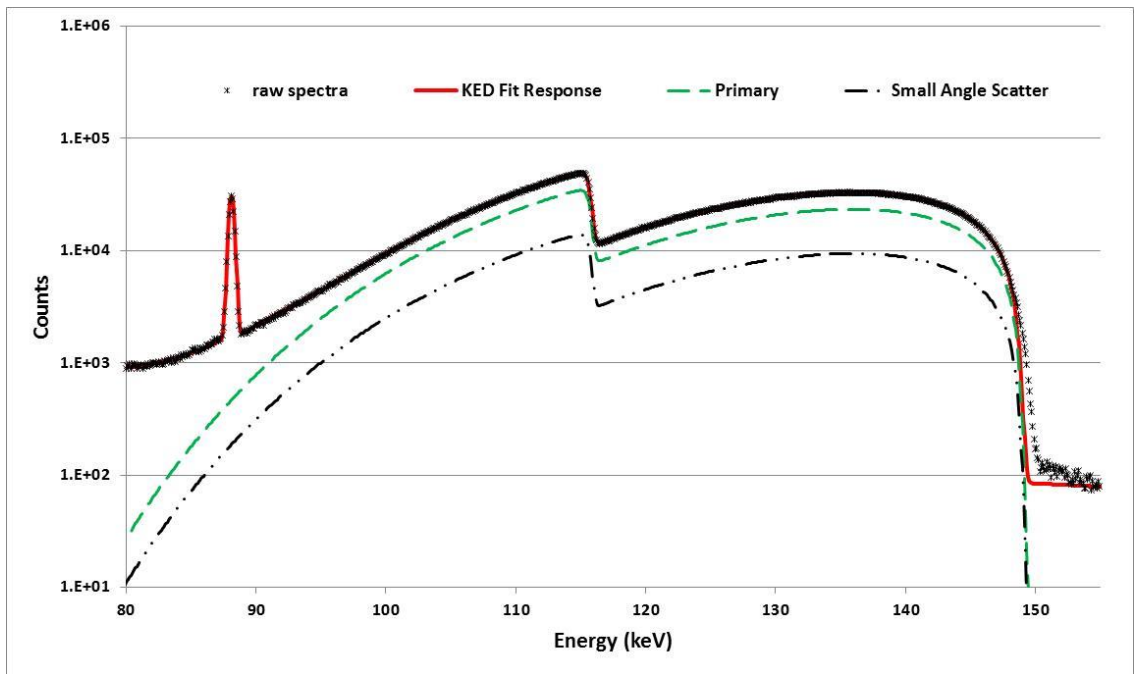

Figure A4. Small angle scattering contribution to the KED transmission spectrum. Note: "Primary" refers to those X-rays transmitted through the sample that are not scattered within the collimator.

\section{Fission Product Background}

Dissolver solutions will contain significant quantities of fission products, and some portion of the gammarays emitted by these radionuclides will be detected. A typical passive spectrum for a dissolver solution (acquired with the X-ray tube off) is shown in Figure A5. Because of the very narrow solid angle of the KED collimator $\left(\sim 10^{-4}\right.$ steradians $)$ and thin HPGe detector $(10 \mathrm{~mm})$, no gamma-ray peaks are evident in the spectrum. However, scattering of the fission product gamma-rays does introduce a featureless background whose parameters are determined during the spectral fitting process.

Note that only a very few KED spectra with fission product loadings were available for this study. The impact on the measurement precision is expected to be small but has not yet been characterized.

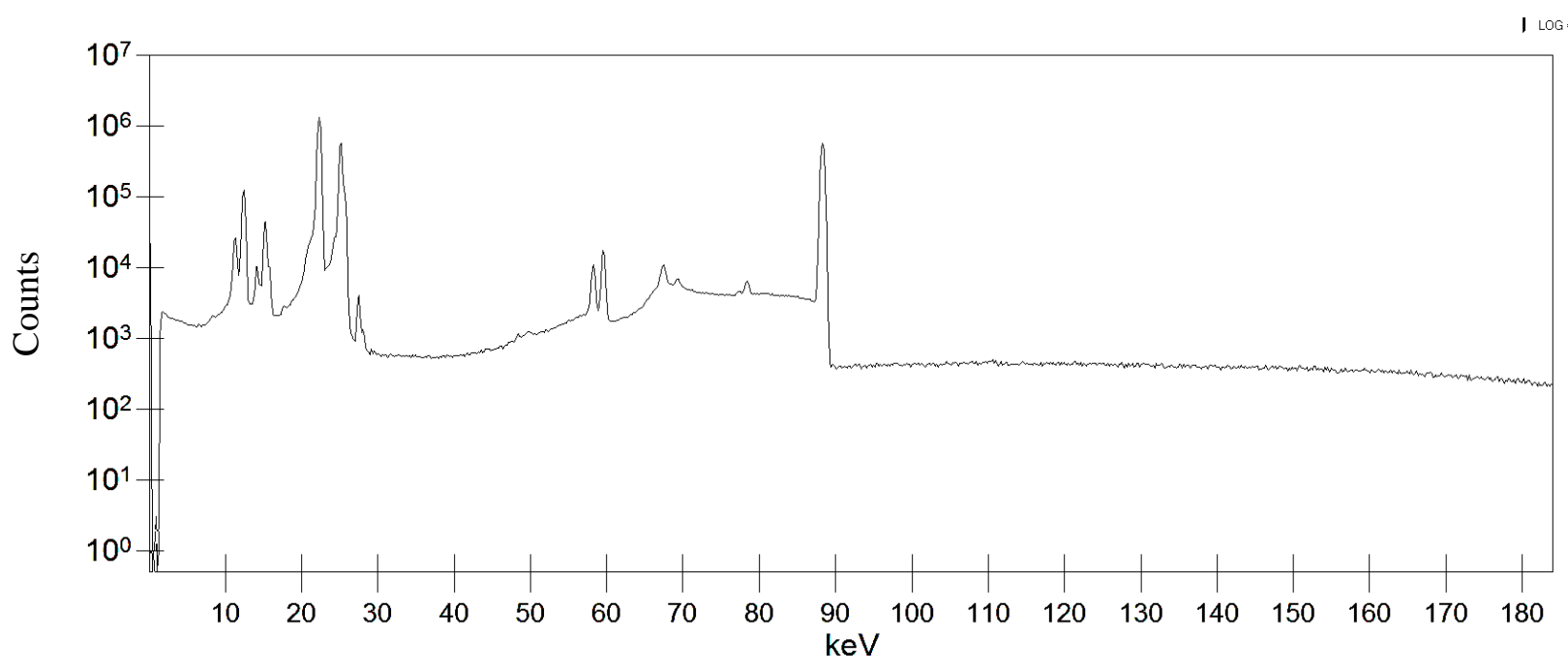

Figure A5. Passive K-Edge transmission measurement spectrum from a fission product-loaded dissolver solution. Note the featureless background over the range of $90-180 \mathrm{keV}$. 


\section{A-3. DETECTOR RESPONSE}

The detector response to a mono-energetic full energy deposition is determined as a function of incident photon energy (Figure A6). The response function is then applied to the calculated transmission distribution described in Section A-2. The HPGe response function includes the following components:

- Gaussian broadening

- Exponential tailing

- Stepped background

- Detection efficiency

- Germanium escape peaks

- Backscatter

- Random coincidence summing

Representative functions for each of the detector response components have been determined and are described in the following sections.

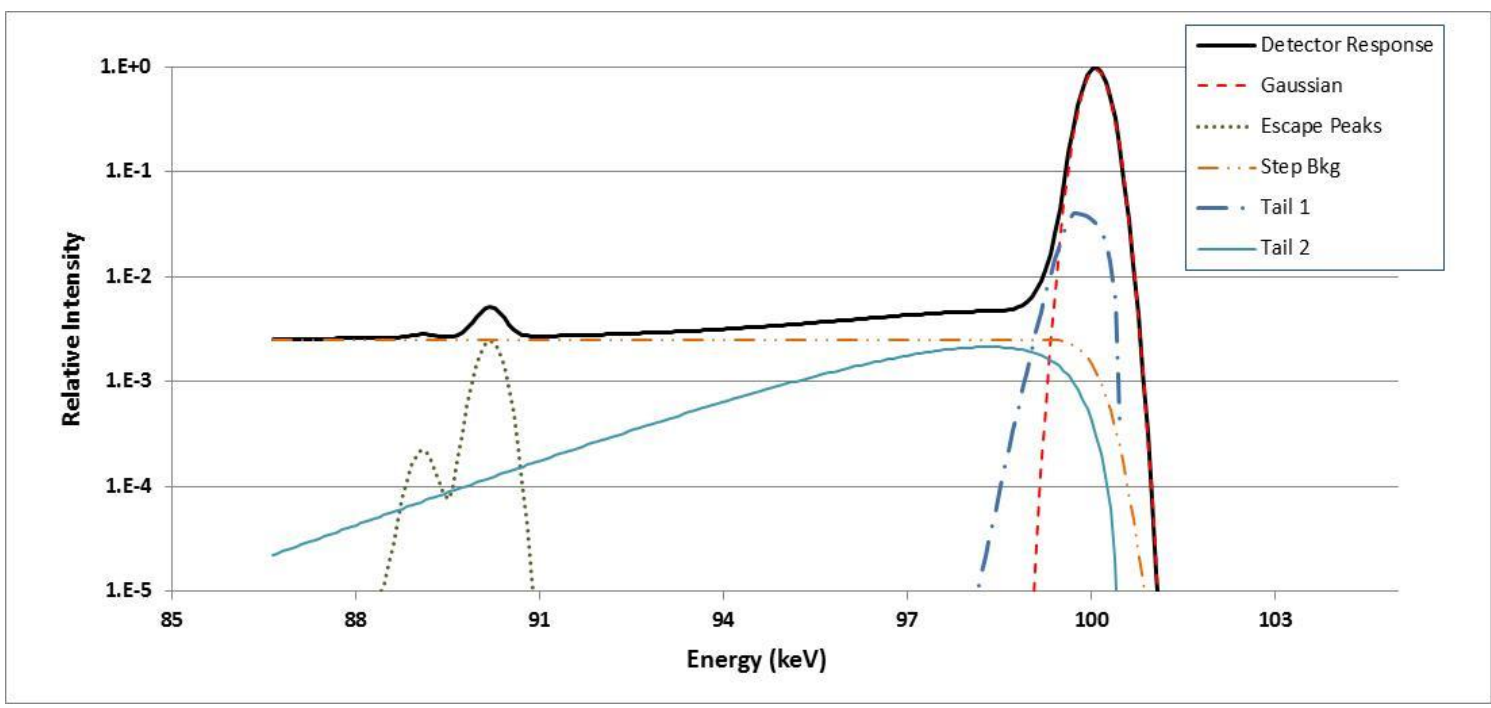

Figure A6. Plot showing the various contributions to the detector response function for a monoenergetic photon source incident normal to the detector surface.

In creating the KED response function, we treat the source term components as if they were a continuous distribution of mono-energetic peaks. To each "peak" the tailing and the step background is added and is broadened corresponding to the observed detector resolution.

\section{Gaussian Broadening}

To reflect the broadening of the spectra based on the response of the detector, the traditional Gaussian broadening is applied

$$
g\left(E, E^{\prime}\right)=\frac{1}{\sqrt{2 \pi} \cdot \sigma} \cdot e^{-\left(E-E^{\prime}\right) / 2 \sigma^{2}}
$$

where $\sigma$ is the standard deviation of the detector resolution,

$E$ is the energy of the source photon,

$E^{\prime}$ is the energy of the detected energy. 


\section{Tail Function}

Peaks in gamma-ray spectra obtained with HPGe (or other detectors) exhibit a low energy tailing feature typically represented as the sum of two exponential components (for example see Gunnink [17]). However, the KED transmission spectrum is not characterized by discrete gamma-ray or X-ray peaks; instead the spectra must be considered as a continuum of peaks and the tailing function applied to the continuous X-ray distribution incident on the detector.

Although Gunnink assumes that the source of the tail is mono-energetic, we instead assume that the tail derives from each part of the full-energy peak; that is, the exponential tail described by Gunnink should be broadened by the Voigt function describing the peak. However, the exponential tail represents a small fraction of the peak area, so for computational efficiency, only the contribution from \pm 1 FWHM about the peak centroid is needed. For the typical HKED spectrum, \pm 1 FWHM $(\approx 2.35 \sigma)$ is equivalent to about 15 channels about the peak. The tail function is given as

$$
f_{\text {tail }}(E)=\int_{E}^{\infty}\left(\omega_{S} \cdot e^{-\kappa_{S} \cdot\left(E^{\prime}-E\right)}+\omega_{F} \cdot e^{-\kappa_{F} \cdot\left(E^{\prime}-E\right)}\right) \cdot d E^{\prime},
$$

where $\omega_{S}$ is the relative intensity of the slow tail,

$\kappa_{S}$ is the decay constant of the slow tail,

$\omega_{F}$ is the relative intensity of the fast tail,

$\kappa_{F}$ is the decay constant of the fast tail,

$\mathrm{E}$ is the energy of the source photon,

$E^{\prime}$ is the energy of the detected energy.

\section{Germanium Escape Peaks}

The germanium escape peaks occur when a germanium X-ray, emitted due to the excitation of the germanium crystal by the incident photon escapes the crystal, reducing the energy available for deposit within the detector. This results in small secondary peaks with 9.88 and $10.99 \mathrm{keV}$ lower energy than the incident photon. However, the escape of germanium X-rays due to the incidence of a continuous spectrum on the crystal creates two lower intensity, continuous spectra similar in shape to the incident spectrum but offset to lower energies. These escape peak spectra are represented by the following:

$$
f_{\text {escape }}(E)=\sum_{i=1}^{2} \omega_{G e, i}(E) \cdot f_{\text {Brem }}\left(E+E_{G e, i}\right) \cdot \delta_{a t t n}\left(E+E_{G e, i}\right) \cdot \delta_{\text {samp }}\left(E+E_{G e, i}\right) \cdot \varepsilon(E),
$$

where $\omega_{G e, i}$ is the intensity of the escape peak relative to the full-energy peak,

$E_{G e, i}$ is the energy of the escaping photon.

The escape peak intensities are dependent on the incident X-ray energy. The intensity for each escape peak is (for energies greater than $40 \mathrm{keV}$ )

$$
\omega_{G e, i}(E) \cong 0.3 \cdot \frac{\mu_{G e\left(E_{i}\right)}}{\mu_{G e}(E)} \cdot I_{i},
$$

where $\mu_{G e\left(E_{i}\right)}$ is the mass attenuation coefficient for germanium $\mathrm{K}_{\alpha}$ energies (i.e., 9.88 and $10.99 \mathrm{keV}$ ), $\mu_{G e(E)}$ is the mass attenuation coefficient for germanium at the actinide photo-peak energy, 
$I_{i}$ is the yield for the two germanium escape peak groups (i.e., 0.474 and 0.0398 , respectively).

The factor of 0.3 is an empirically determined factor for the specific detector/measurement arrangement. A plot of the energy dependence of the germanium escape peak intensity is shown in Figure A.7.

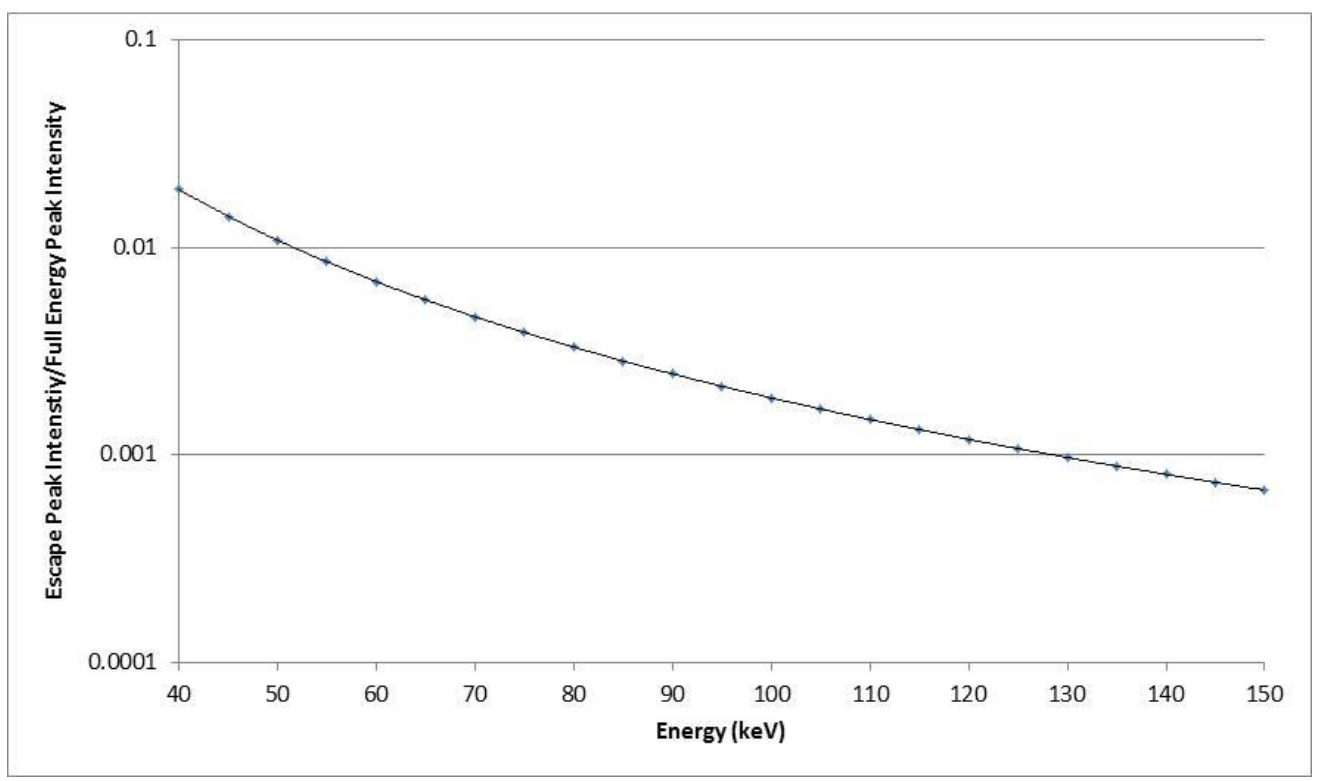

Figure A7. Plot of relative germanium escape peak intensity as a function of photo-peak energy.

\section{Step Background $b_{\text {step }}(E)$}

The KED transmission spectrum includes a "step" background component originating from the incomplete detection of the X-rays [17]. In traditional gamma-ray spectroscopy the step background is essentially a third tail component but one with decay constant equal to zero. This can be represented by the following

$$
b_{\text {step }}(E)=\int_{E}^{E_{\max }} w_{\text {step }} \cdot f_{\text {Brem }}\left(E^{\prime}\right) \cdot \delta_{\text {attn }}\left(E^{\prime}\right) \cdot \delta_{\text {samp }}\left(E^{\prime}\right) \cdot \varepsilon\left(E^{\prime}\right) \cdot d E^{\prime}
$$

where $w_{\text {step }}$ is the step background magnitude and is empirically determined during the fit to the spectra,

$E_{\max }$ is the X-ray generator end-point energy.

Alternatively, it is possible to adopt a modification of the traditional step background correction implemented by Ottmar and Eberle [1]. The step background implemented in Reference [1] assumes the background at energy, $E$, is proportional to the sum of counts within the spectrum at energies greater than $E$ divided by the sum of all counts between two background regions of interest (ROIs) $\left(b_{\text {lower }}\right.$ and $\left.b_{\text {upper }}\right)$ at energies $E_{\text {lower }}$ and $E_{\text {upper }}$ :

$$
b_{\text {step }}(E)=b_{\text {upper }}+\frac{\sum_{E}^{E_{\text {upper }}} \mathrm{C}(\mathrm{E})}{\sum_{E_{\text {lower }}}^{E_{\text {upper }}} \mathrm{C}(\mathrm{E})} \cdot\left(b_{\text {lower }}-b_{\text {upper }}\right)
$$


The position of the upper and lower ROIs is shown in Figure A.8. However, the lower background region typically used for the KED measurement (61.57-64.54 keV) includes a contribution from the tungsten Xrays fluoresced by the ${ }^{109} \mathrm{Cd}$ reference source gamma-rays (Figure A9), and the upper background region includes a contribution from random coincidence scattering. This leads to an overcorrection for the step background that also varies in time with the decay of the ${ }^{109} \mathrm{Cd}$ source. To correct the overestimate, we determine the step background after the random coincidence contribution has been removed and adjust the lower region for the ${ }^{109} \mathrm{Cd}$ induced $\mathrm{X}$-rays.

$$
b_{\text {step }}(E)=b_{\text {upper }}^{\prime}+\frac{\sum_{E}^{E_{\text {upper }}} \mathrm{C}(\mathrm{E})-f_{r_{\text {sum }}}(\mathrm{E})}{\sum_{E_{\text {lower }}}^{E_{\text {loper }}} \mathrm{C}(\mathrm{E})-f_{r_{\text {sum }}}(\mathrm{E})} \cdot\left(b_{\text {lower }}^{\prime}-f_{C d} \cdot I_{C d}-b_{\text {upper }}^{\prime}\right)
$$

where $b^{\prime}$ lower is the random coincidence corrected counts in the lower ROI, $b^{\prime}{ }_{\text {upper }}$ is the random coincidence corrected counts in the upper ROI, $I_{C d}$ is ${ }^{109} \mathrm{Cd}$ peak counts, $f_{C d}$ is the ratio of ${ }^{109} \mathrm{Cd}$ induced X-rays in the lower ROI to ${ }^{109} \mathrm{Cd}$ peak counts.

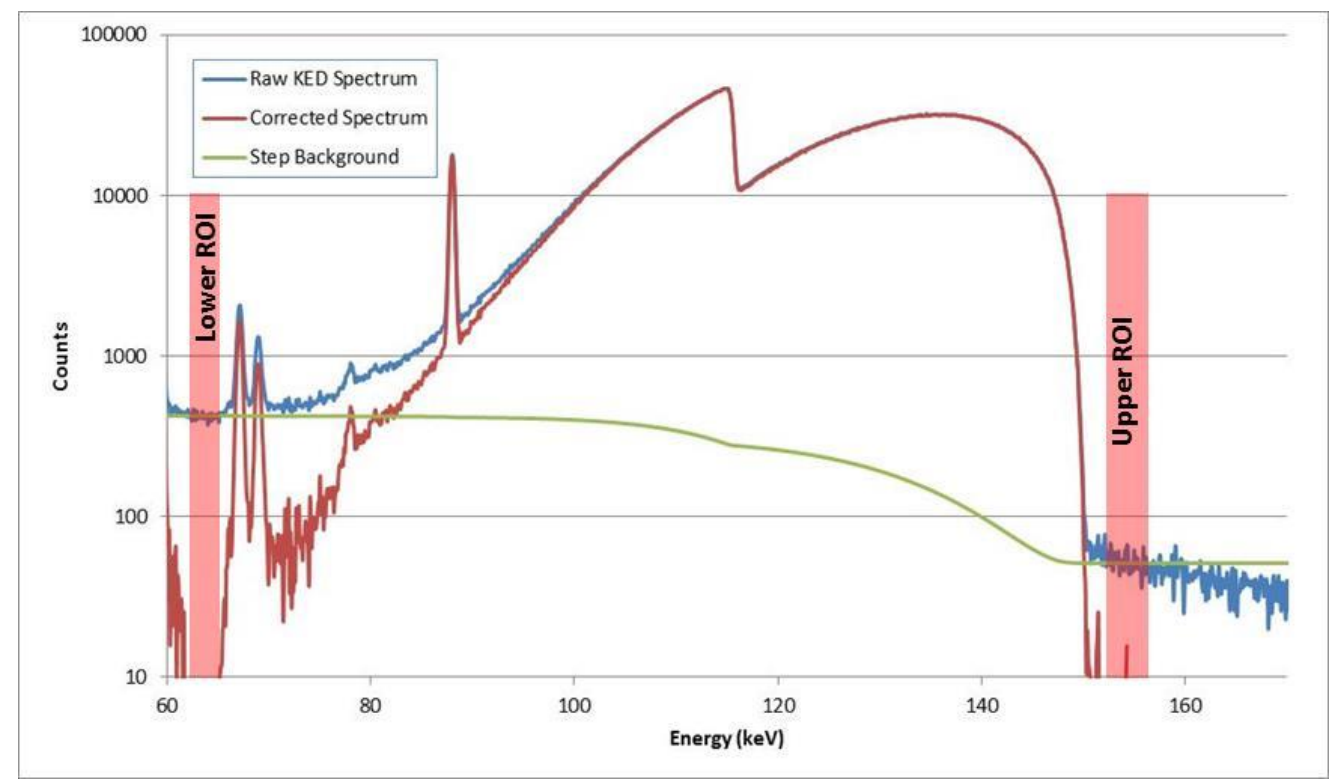

Figure A8. Step background determined for a $321.9 \mathrm{~g}$ U/L solution as implemented in Ref. [1].

Unfortunately, these step background tails do not extend to zero energy but have a finite and variable extent of only 10-20 keV in the energy range of interest. The fact that in measured spectra these tails do not extend to zero energy is not discussed in the references [17], [18], [19] where the step background is usually only examined over a small energy interval close to the full energy peak. However, in our case the energy window of interest from 85 to $150 \mathrm{keV}$ is much longer than the typical tail length. A more accurate representation of the step background is under investigation. 


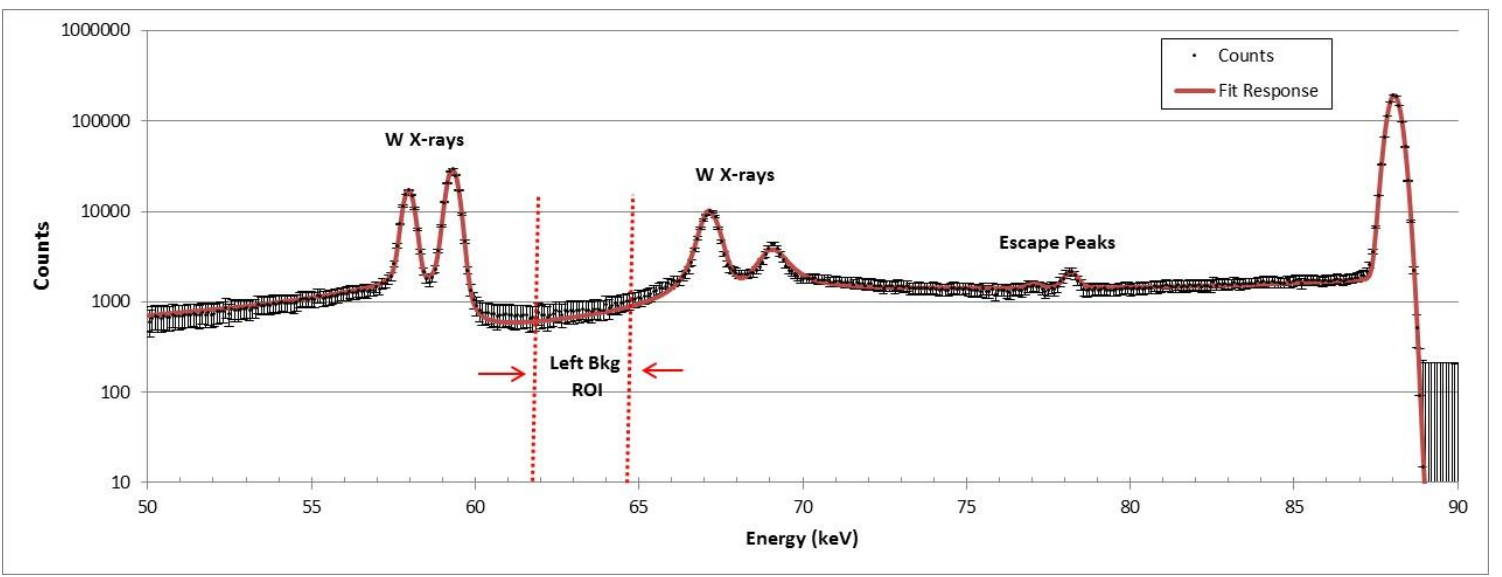

Figure A9. Plot of the passive (X-ray off) KED spectrum showing the contribution to the lower ROI from the ${ }^{109} \mathrm{Cd}$ source (acquisition time $=40,000 \mathrm{~s}$, peak rate $\sim 27 \mathrm{cps}$ ).

\section{Detection Efficiency}

The absolute detection efficiency has no direct bearing on the analysis; however, a reasonable representation of the relative energy response is necessary to determine the magnitude of the transmission change across the K-Edge. The detection efficiency as a function of energy, $\varepsilon(E)$, is represented by an empirically determined function. The detection efficiency of the HPGe detectors can be described by a fifth-order polynomial

$$
\varepsilon(E)=\sum_{i=1}^{7} \varphi_{i} \cdot q^{i-1},
$$

where $q=\ln \left(\varphi_{7} / E\right)$,

$\varphi_{1}$ to $\varphi_{7}$ are empirically determined coefficients.

The variation in full energy peak efficiency over the range of $85-150 \mathrm{keV}$ is only a few percent, so it is not necessary to directly measure the efficiency for the HKED system. Computational efficiency determination (e.g., MCNP [20] or ISOCS [21]) is sufficiently accurate to correct for this relatively modest and smooth efficiency variation. We used the ISOCS software for convenience.

The model used for computation of the efficiency is based on the planar detector (Canberra LEGE0210) with thin epoxy window, tungsten collimator, and stainless-steel beam filter, the stainless-steel wall of the HKED transfer tube, and the sample vial polyethylene wall. The results of that model yield the following parameters to describe the efficiency of the XRF detector assembly (Table A2).

Table A2. Representative parameters for the XRF detection efficiency

\begin{tabular}{ll}
\hline$\varphi_{1}=-7.26$ & $\varphi_{5}=0.4638$ \\
$\varphi_{2}=0.8296$ & $\varphi_{6}=-0.1317$ \\
$\varphi_{3}=-0.5733$ & $\varphi_{7}=160 \mathrm{keV}$ \\
$\varphi_{4}=-0.3383$ & \\
\hline
\end{tabular}




\section{Detector Backscatter Component}

The HPGe detector crystal is thin to minimize sensitivity to energetic fission product gamma-rays (e.g. ${ }^{137} \mathrm{Cs}$ ). However, the thinness of the crystal allows transmission of a fraction of the photons incident on the detector face through the crystal. The transmitted photons can scatter inelastically from the copper cold finger located behind the crystal back into the detector where they are detected with reduced energy. A plot of the backscatter contribution to the KED transmission spectrum is shown in Figure A10. It is apparent in the plot that the highest energy scattered photon is well below the U K-Edge transition energy, however, it is necessary to include this contribution to achieve a reliable fit response for the KED analysis. It will also be necessary to account for this scattering should thorium or lower $\mathrm{Z}$ actinides be included within the sample.

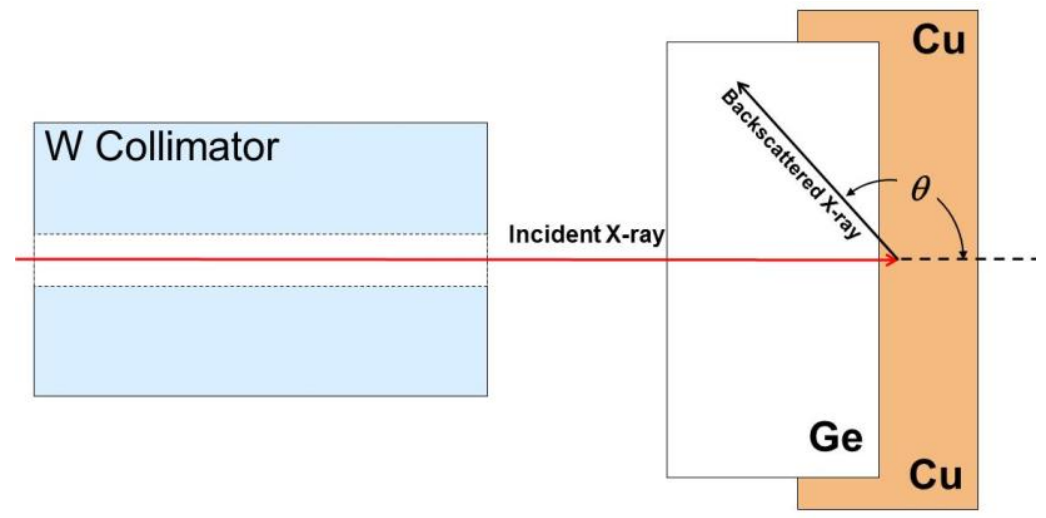

Figure A10. Cartoon illustrating the frame of reference for the backscattered photons.

The energy available for the backscattered photon, $E$, varies as a function of the backward scattering angle, $\theta$, and incident photon energy, $E^{\prime}$, is given by the Klein-Nishina relation,

$$
\begin{gathered}
\boldsymbol{E}=\frac{\boldsymbol{E}^{\prime}}{\mathbf{1}+\frac{\boldsymbol{E}^{\prime}}{\boldsymbol{m}_{\boldsymbol{e}}}(\mathbf{1}-\boldsymbol{c o s}(\boldsymbol{\theta}))} \\
f_{\text {back }}(E)=\int_{E}^{E_{\max }^{\prime}} C(E) \cdot f_{r e l}\left(E^{\prime}\right) \cdot\left[f_{s}^{2} \cdot r_{e} \cdot P(E)^{2} \cdot\left[P(E)+\frac{1}{P(E)}-\sin ^{2}(\theta)\right] / 2\right] \cdot d E^{\prime}
\end{gathered}
$$

The impact of attenuation of the incident photon (with energy E') by its passage through the HPGe crystal and relative detection efficiency of scattered photon (with energy $\mathrm{E}$ ), $f_{r e l}(E)$, is given by

$$
f_{r e l}\left(E^{\prime}\right)=e^{-\mu_{G e\left(E^{\prime}\right)} \cdot \rho_{G e} \cdot d_{G e}} /\left(1-e^{-\mu_{G e(E)} \cdot \rho_{G e} \cdot d_{G e}}\right)
$$


where $E$ is the energy of the backscattered photon,

$E^{\prime}$ is the energy of the photon incident on the detector face,

$E_{\text {max }}^{\prime}$ is the highest energy photon incident on the detector face capable of producing a backscatter photon of energy E, ${ }^{1}$

$P(E)=1 / 1+E^{\prime} / m_{e} \cdot(1-\cos (\theta))$,

$\theta=\cos ^{-1}\left(1-\left(\frac{E^{\prime}}{E}-1\right) \cdot \frac{m_{e}}{E^{\prime}}\right)$,

$\rho_{G e}$ is the density of germanium $(5.323 \mathrm{~g} / \mathrm{cc})$,

$\mu_{G e}$ is the mass attenuation coefficient of germanium,

$d_{G e}$ is the thickness of the HPGe detector crystal (typically $d_{G e}=10 \mathrm{~mm}$ ),

$\mathrm{f}_{\mathrm{s}}=1 / 137.04$ (the fine structure constant),

$r_{e}=\hbar / m_{e} \cdot c=0.38616 \cdot 10^{-12} \mathrm{~m}$,

$m_{e}$ is the rest mass of the electron in $\mathrm{keV}$,

$\theta=$ scattering angle,

$\theta_{\min }=$ the minimum scattering angle.

The relative contribution to the transmission spectrum due to the backscatter component is illustrated in Figure A11 for a solution containing $100 \mathrm{~g} \mathrm{U} / \mathrm{L}$.

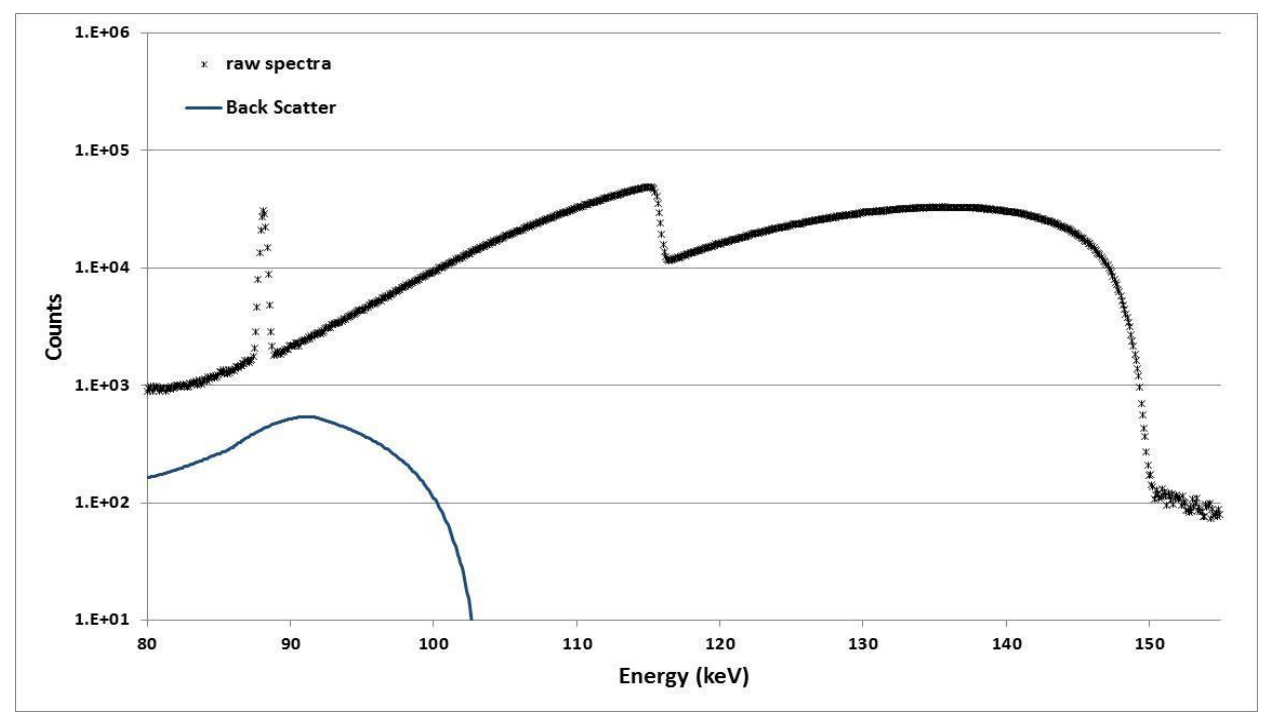

Figure A11. Detector backscatter contribution to the KED transmission spectrum.

An alternative treatment of the backscattering component is to strip the scattering component from the raw spectrum. This is in some ways a simpler approach because the measured spectrum already includes detector response and attenuation effects. In this case, we calculate a scattered spectrum for the highest energy channel in the measured spectrum, subtract that spectrum from the raw data, and then repeat for each of the lower energy channels.

\section{Cadmium-109 Gamma-ray Peak Area}

The $88.034 \mathrm{keV}$ line from the ${ }^{109} \mathrm{Cd}$ reference source is included within the KED fitting region to provide a reliable energy reference for the transmission spectrum. Because we are now dealing with a traditional

\footnotetext{
${ }^{1} \mathrm{E}_{\max }$ is produced at the minimum backscatter angle for a photon to interact within the detector. From Figure A11 we expect that $\theta_{\text {min }}$ should be $\cong 90^{\circ}$; however, dead layers at the back of the detector will increase this angle. For the Canberra GL0210P detector, $\theta_{\min }$ is estimated to be $\sim 115^{\circ}$.
} 
mono-energetic gamma-ray emission line, the traditional peak, tail, and step background representations are used [17]:

$$
\begin{gathered}
I_{C d}(E)=\frac{I_{C d}}{\sqrt{2 \pi} \cdot \sigma} \cdot e^{-\left(E-E_{0}\right)^{2} / 2 \cdot \sigma^{2}}+f_{\text {tail }}(E)+f_{\text {escape }}(E)+b_{\text {step }}(\mathrm{E}) \\
f_{\text {tail }}(E)=I_{C d} \cdot\left[\omega_{S} \cdot e^{-\kappa_{S} \cdot\left(E^{\prime}-E\right)}+\omega_{F} \cdot e^{-\kappa_{F} \cdot\left(E^{\prime}-E\right)}\right] \cdot\left(1-e^{0.4 \cdot\left(E^{\prime}-E\right)^{2} / 2 \cdot \sigma^{2}}\right) \\
f_{\text {step }}(E)=I_{C d} \cdot w_{\text {step }} \cdot\left[1-\operatorname{erf}\left(\left(E-E^{\prime}\right) / 2 \cdot \sigma\right)\right]
\end{gathered}
$$

\section{Random Coincidence Summing}

The HKED system is operated at high counting rates (15-80 kcps) in relation to pulse processing times resulting in non-negligible random coincidence summing where two low-pulse height events occur at the same time forming a larger pulse. As an example, the KED count rate for the reference nitric acid solution (Figure A9) should monotonically decrease for energies greater than $\sim 80 \mathrm{keV}$ and reach zero as the energy approaches the X-ray tube endpoint energy $(150 \mathrm{keV})$. Instead there is a significant count rate observable at energies greater than the HV endpoint. These excess counts are due to random coincidence summing.

The random coincidence summing creates an interfering spectrum from the non-coincidence spectra convoluted with itself. For the reference spectra, with no actinides present, the sum spectrum is essentially featureless over the energy range of interest ( 80 to $150 \mathrm{keV}$ ), as shown in Figure A12 while some structure is apparent in the samples containing higher concentrations of actinides as shown in Figure A13. To resolve the coincidence and non-coincidence contributions to the spectra, a spectral unfolding routine would normally need to be employed; however, the energy distribution of the KED spectra allows us to approximate the random sum spectra over the energy range of interest by convoluting the measured spectrum with itself. A tailing function is added to the resulting spectra to account for the nonideal peak shapes arising from the accidental coincidence summing process. The random coincidence summing rate, $r_{j}$, is given by

$$
r_{j}=\frac{G}{t_{L}} \cdot \sum_{i=1}^{j-1} C_{i} \cdot C_{j-i}
$$

where $r_{j}$ is the coincidence rate in channel $j$,

$C_{i}$ is the counts in $\mathrm{i}^{\text {th }}$ channel,

$G$ is the coincidence time parameter (empirically determined for each HKED system, $\sim 0.55 \mu \mathrm{s}$ ), $t_{L}$ is the live time for the spectrum.

The coincidence rate for the $j^{\text {th }}$ channel of the spectrum adjusted for tailing, $R_{j}$, is given by

$$
R_{j}=1+\sum_{n=1}^{n_{\max }} a_{R S} \cdot e^{-\left(\mathrm{n} \cdot \Delta_{E} \cdot \lambda_{R S}\right)} \cdot r_{j+n}
$$

where $R_{j}$ is the random coincidence count in channel $\mathrm{j}$ with tailing added,

$a_{R S}$ is tail function area,

$\lambda_{R S}$ is the decay constant of the exponential tailing function,

$n$ is the number of channels away from the channel $\mathrm{j}$,

$\Delta_{E}$ is the energy per channel of the spectrum. 


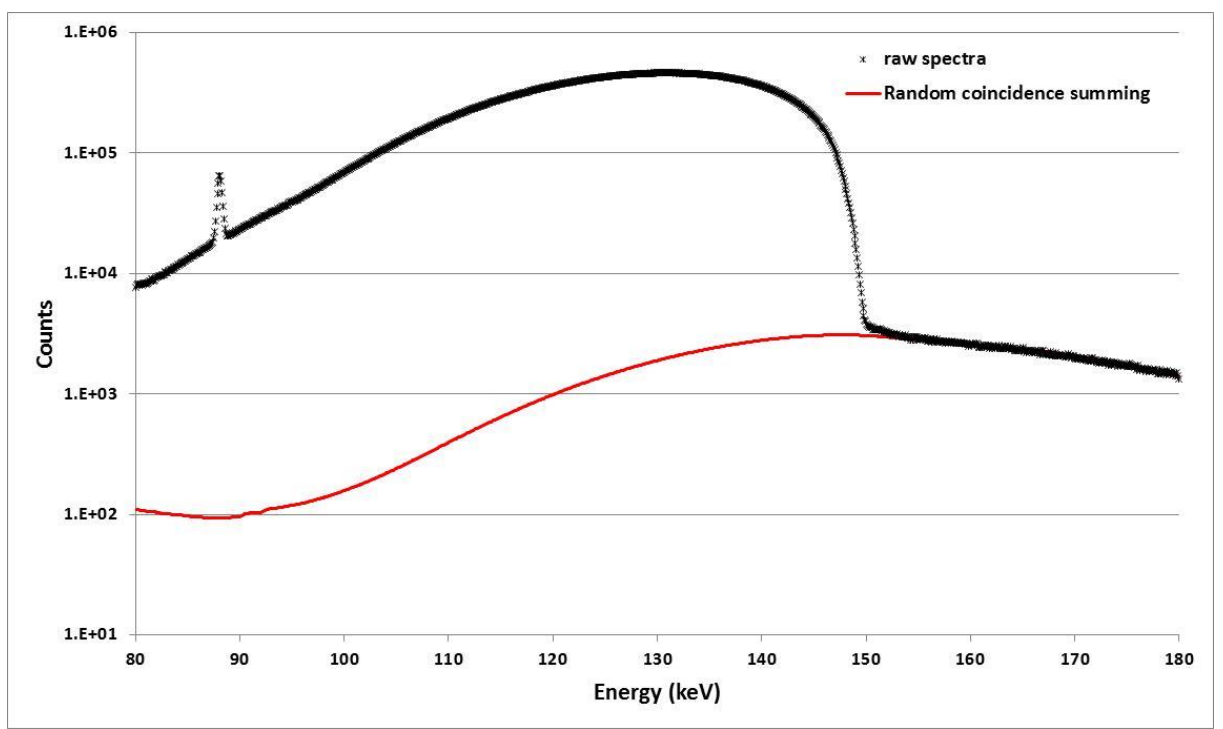

Figure A12. Plot of the high-energy portion of the KED spectrum from a $3 M$ nitric acid reference solution. The random sum spectrum (the convolution of the spectrum on itself) is normalized to the uncorrected spectrum over the energy range of $155-170 \mathrm{keV}$.

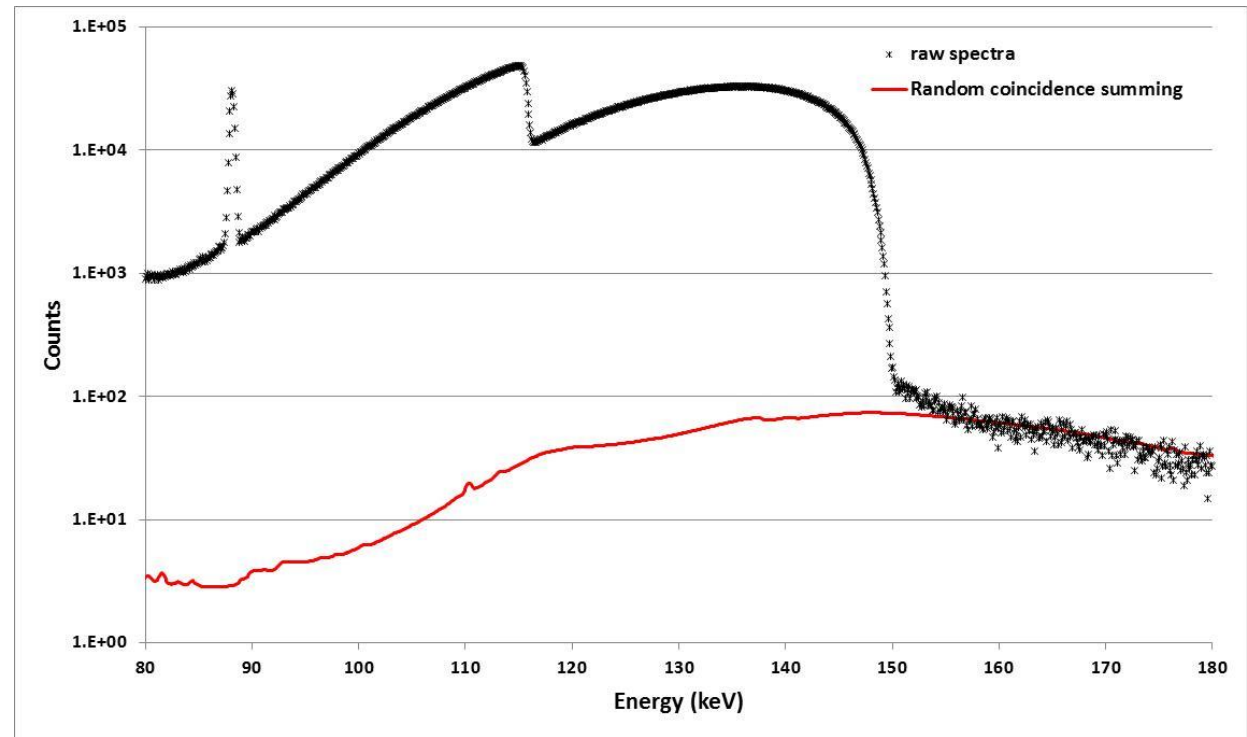

Figure A13. Plot of the high-energy portion of the KED spectrum from a $322 \mathrm{~g}$

$\mathrm{U} / \mathrm{L}$ in $\mathbf{3} M$ nitric acid standard solution. The random sum spectrum (the convolution of the spectrum on itself) is normalized to the uncorrected spectrum over the energy range of $155-170 \mathrm{keV}$.

\section{A-4. MASS ATTENUATION COEFFICIENTS}

The mass attenuation coefficients are derived from the online XCOM database [5]. The mass attenuation coefficients were extracted over the range of $10-1,000 \mathrm{keV}$ and were fit to an analytical form for convenience. The mass attenuation coefficients are represented over the energy range of interest by the following expressions 


$$
\begin{gathered}
\mu_{\text {below }}(E)=10^{\left(\mu_{L 0} \cdot \log (E)^{3}+\mu_{L 1} \cdot \log (E)^{2}+\mu_{L 2} \cdot \log (\mathrm{E})+\mu_{L 3}\right)} \\
\mu_{\text {above }}(E)=10^{\left(\mu_{H 1} \cdot \log (E)^{2}+\mu_{H 2} \cdot \log (\mathrm{E})+\mu_{H 3}\right)}
\end{gathered}
$$

where $\mu_{\text {below }}$ represents the mass attenuation coefficient for energies below the K-Edge transition; $\mu_{\text {above }}$ represents the mass attenuation coefficient for energies above the K-Edge transition; $\mu_{L 1}, \mu_{L 2}, \mu_{L 3}$ are the fitted coefficients for the expression representing $\mu_{\text {below }}$; $\mu_{H 1}, \mu_{H 2}, \mu_{H 3}$ are the fitted coefficients for the expression representing $\mu_{\text {above }}$.

The XCOM database does not include any broadening of the mass attenuation coefficients near the KEdge transition due to the natural line width of the reaction. As illustrated in Figure A14, the transition as described by the XCOM database is represented by a step function. The parameters for the analytic representation of the mass attenuation coefficients for the actinides of interest are provided in Table A3.

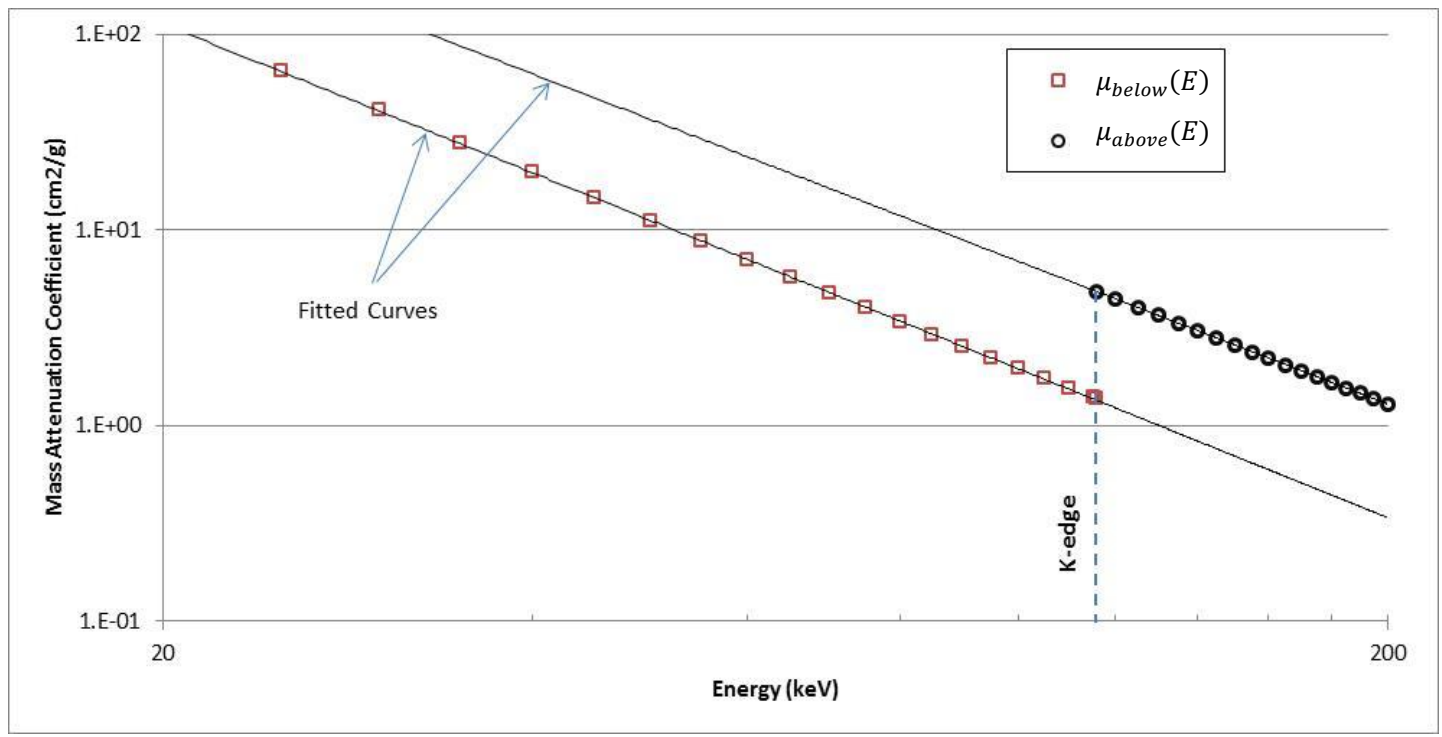

Figure A14. Uranium mass attenuation coefficient (with coherent scattering) as a function of energy near the K-Edge transition. Data points shown were obtained from reference [5].

Table A3. Mass attenuation coefficient parameters (including coherent scattering) based on fits to the XCOM data over the energy range of 45 to $200 \mathrm{keV}$.

\begin{tabular}{ccccccc}
\hline & Uranium & Neptunium & Plutonium & Americium & Curium & $3 \mathrm{M} \mathrm{HNO}_{3}$ \\
\hline$\mu_{L 0}$ & 0 & 0 & 0 & 0 & 0 & - \\
$\mu_{L 1}$ & 0.08624 & 0.0583 & 0.08396 & 0.0880 & 0.0795 & - \\
$\mu_{L 2}$ & -2.82883 & -2.722 & -2.81171 & -2.8207 & -2.786 & - \\
$\mu_{L 3}$ & 5.60467 & 5.5244 & 5.61750 & 5.6318 & 5.6087 & - \\
\hline$\mu_{H 1}$ & 0.18565 & 0.1941 & 0.18575 & 0.1645 & 0.088 & 0.1180 \\
$\mu_{H 2}$ & -3.23248 & -3.2653 & -3.22297 & -3.1239 & -2.7804 & -0.8204 \\
$\mu_{H 3}$ & 6.56833 & 6.6167 & 6.577995 & 6.4687 & 6.0914 & 0.3958 \\
\hline K-Edge (keV) [22] & 115.602 & 118.699 & 121.791 & 124.982 & 128.241 & - \\
\hline Line width (eV) [23] & 96.3 & 100.5 & 104.4 & 108.2 & 112 & - \\
\hline
\end{tabular}


However, incorporation of these attenuation coefficients into the KED response function does not provide a satisfactory representation of the observed spectra. Figure A15 provides a comparison of the fitted response with the measured spectrum for a uranyl-nitrate solution with a concentration of $322 \mathrm{~g} \mathrm{U} / \mathrm{L}$. Although the fitted response has been smoothed for the detector response function (Gaussian broadened and tailing function added), the predicted transition at the K-Edge is much sharper than is observed.

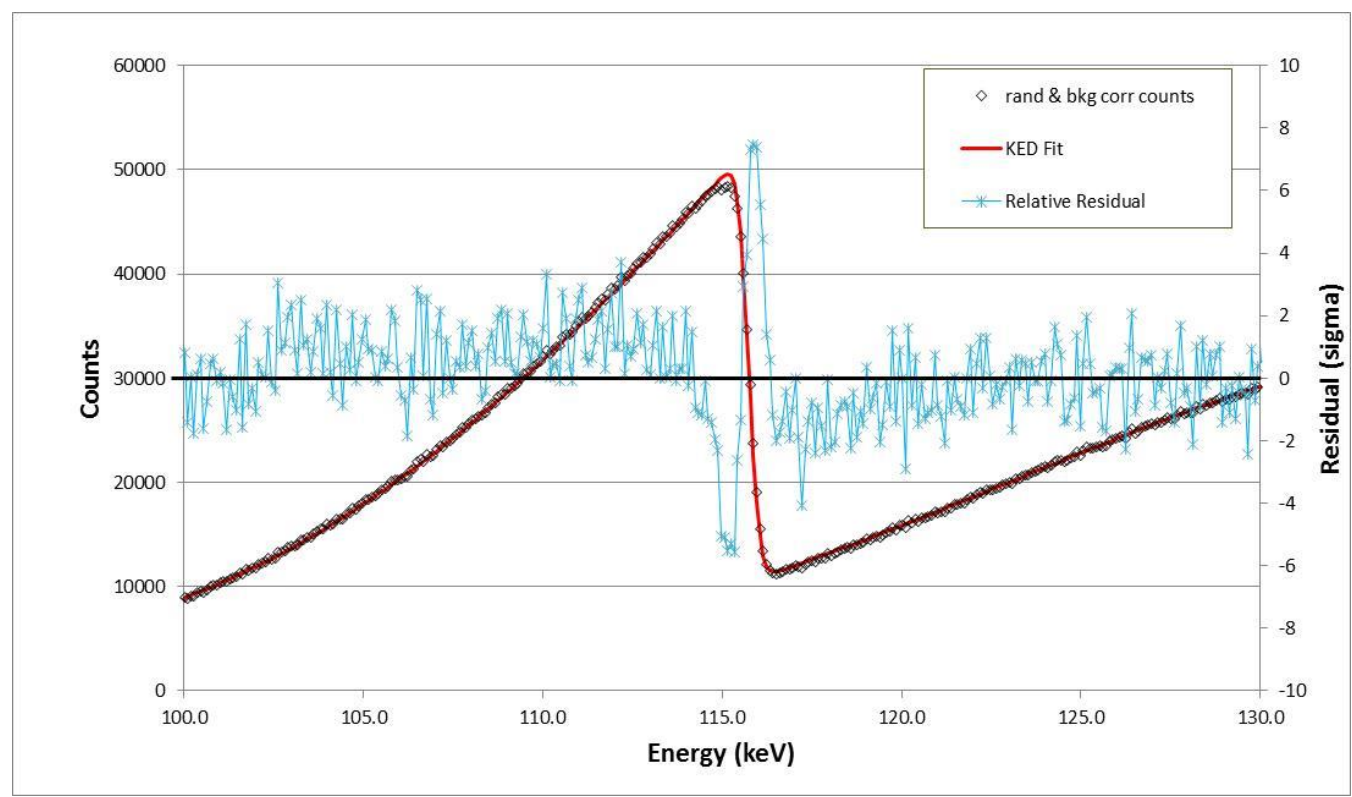

Figure A15. Fit to a KED spectrum obtained from a solution containing approximately $322 \mathrm{~g}$ U/L, assuming no Lorentzian broadening of the K-Edge transition. The plot shows the fit to the measured spectrum after correction for background and random coincidence summing. The relative residuals are overlain in terms of multiple standard deviations and illustrate the fidelity of the KED fitting process.

We expect the K-Edge transition to be broadened as the natural line width observed during the emission of the $\mathrm{K}_{\alpha} \mathrm{X}$-rays from the same element [14] [16] [24]. An expression for the mass attenuation coefficient with Lorenztian broadening is given to high approximation as

$$
\mu(E)=\mu_{\text {below }}(E)+\left(\mu_{\text {above }}(E)-\mu_{\text {below }}(E)\right) \cdot\left(\frac{1}{2}+\operatorname{atan}\left(2 \cdot\left(E-E_{\text {Kedge }}\right) / \lambda\right)\right.
$$

where $\lambda$ is the width of the Lorentzian distribution.

However, as can be seen in Figure A16, this representation also fails to adequately represent the transition. And we note that with full Lorentzian broadening of the K-Edge transition the fit response fails for energies just above the K-Edge transition, but without the broadening (Figure A15) the fit fails for energies just below the transition. 


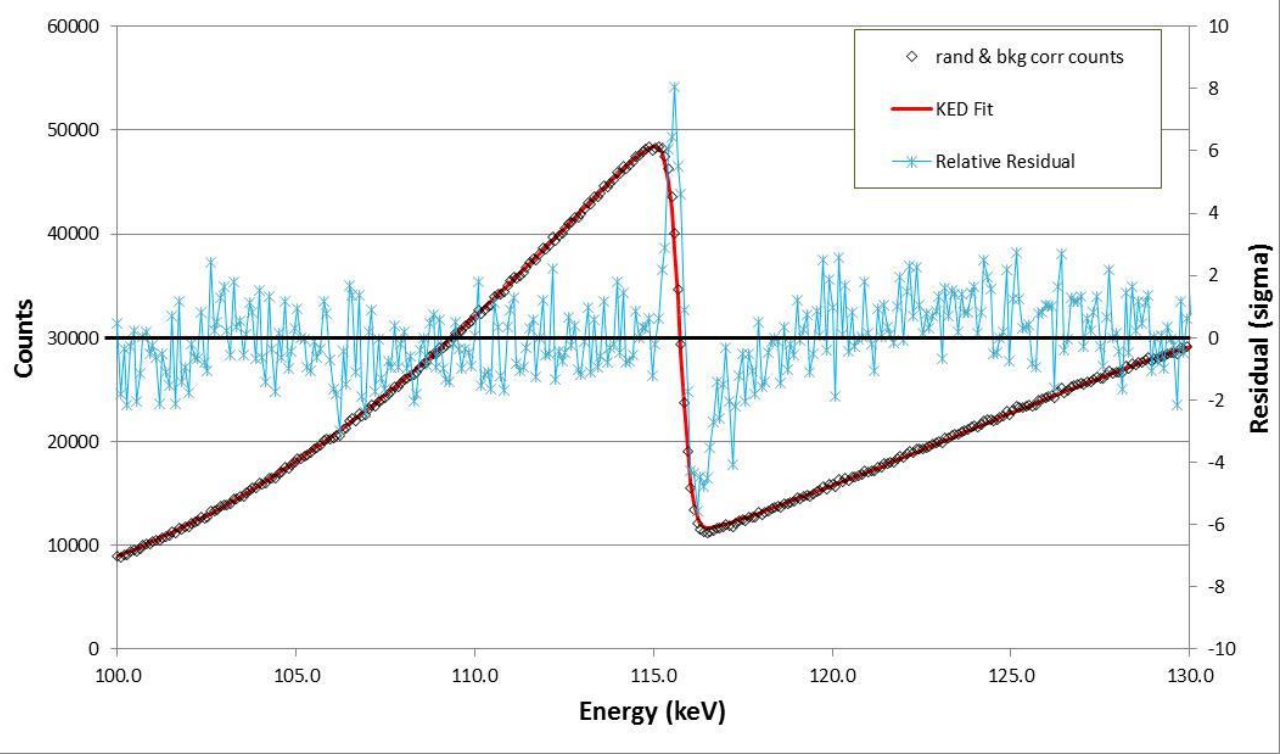

Figure A16. Fit to a KED spectrum obtained from a solution containing approximately $322 \mathrm{~g}$ U/L assuming full Lorentzian broadening of the K-Edge transition. The plot shows the fit to the measured spectrum after correction for background and random coincidence summing. The relative residuals are overlain, in terms of multiple standard deviations, and illustrates the fidelity of the KED fitting process.

We conclude that to accurately represent the K-Edge transmission response function, it is necessary to apply an empirical approach where the mass attenuation coefficient function is Lorentzian broadened only for energies below the transition energy. This may be due to the presence of unresolved fine structure in transmission cross-sections or simply a lack of a detailed reaction model for K-shell emission. The empirical relationship best describing the observed response is given by the following equation and a comparison with measured data shown in Figure A17.

$$
\begin{aligned}
& \mu(E)=\quad \mu_{\text {below }}(E)+\left(\mu_{\text {above }}(E)-\mu_{\text {below }}(E)\right) \cdot\left(\frac{1}{2}+\operatorname{atan}\left(2 \cdot\left(E-E_{\text {Kedge }}\right) / \lambda\right): E<E_{\text {Kedge }}\right. \\
& \mu_{\text {above }}(E) \\
& \text { : } E \geq E_{\text {Kedge }}
\end{aligned}
$$

Although this response function has been empirically validated during the course of this study, why this works is currently an unresolved research question that requires improved basic atomic data to address. 

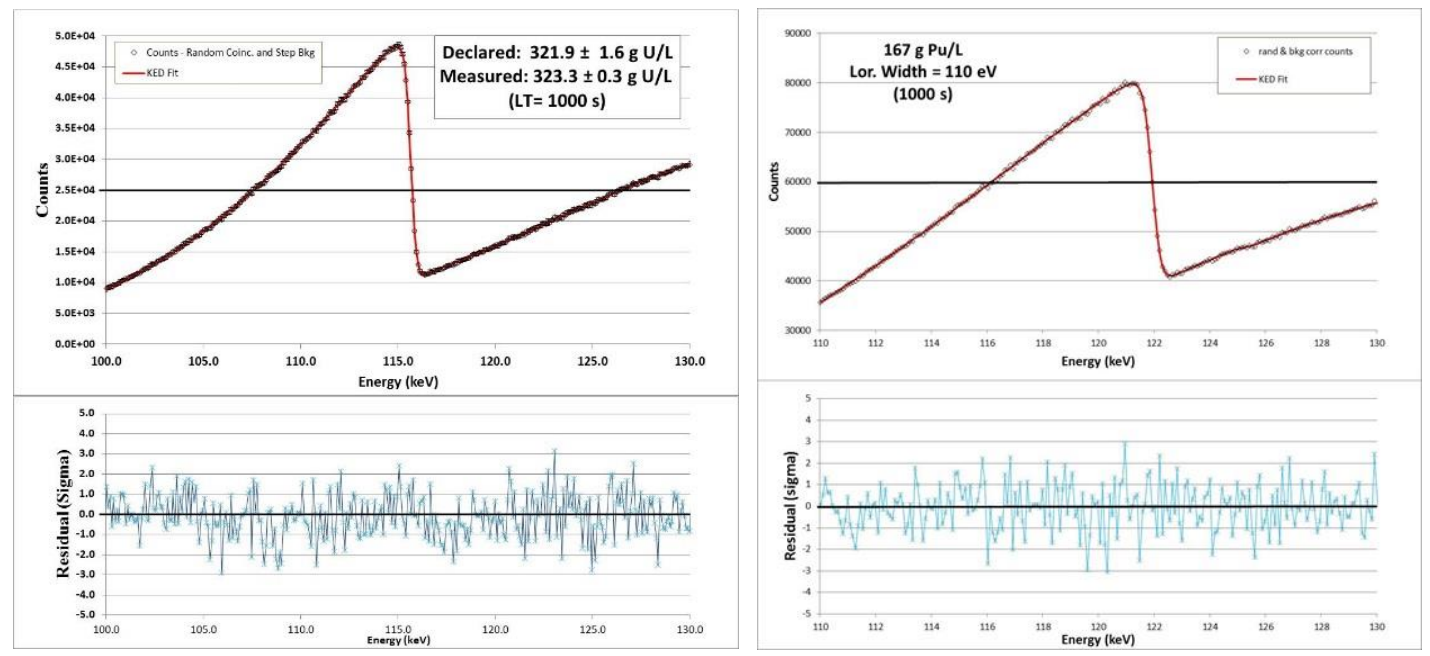

Figure A17. Fit to two KED spectra obtained from a solution containing approximately $322 \mathrm{~g} \mathrm{U} / \mathrm{L}$ (left) and a $167 \mathrm{~g} \mathrm{Pu} / \mathrm{L}$ (right) assuming Lorentzian broadening only of the low energy portion of the K-Edge transition. The upper portion of each plot shows the fit to the measured spectrum after correction for background and random coincidence summing. The lower portion of the plots show the relative residuals, in terms of multiple standard deviations, and illustrates the fidelity of the KED fitting process.

\section{A-5. DETERMINATION OF THE MASS ATTENUATION COEFFICIENTS USING THE KED TRANSMISSION SPECTRA}

We have examined KED transmission spectra from four different HKED systems, two based on the SGN sample vial and two using the composite sample vial described in Reference [6]. In each case we have observed a consistent bias in the reported uranium and plutonium concentrations of $1.1 \%$ and $1.8 \%$, respectively. We have adjusted the mass attenuation coefficients for uranium and plutonium to provide a more accurate assay result and added an additional term for plutonium to more accurately reproduce the energy dependence of the attenuation coefficients.

$$
\begin{aligned}
& \mu_{\text {below }}(E)=10^{\left(\mu_{l 0} \cdot \log (E)^{3}+\mu_{l 1} \cdot \log (E)^{2}+\mu_{l 2} \cdot \log (E)+\mu_{l 3}\right),} \\
& \mu_{\text {above }}(E)=10^{\left(\mu_{h 1} \cdot \log (E)^{2}+\mu_{h 2} \cdot \log (\mathrm{E})+\mu_{h 3}\right),}
\end{aligned}
$$

where $\mu_{\text {below }}$ represents the mass attenuation coefficient for energies below the K-Edge transition;

$\mu_{\text {above }}$ represents the mass attenuation coefficient for energies above the K-Edge transition;

$\mu_{l 1}, \mu_{l 2}, \mu_{l 3}$ are the fitted coefficients for the expression representing $\mu_{b e l o w}$;

$\mu_{h 1}, \mu_{h 2}, \mu_{h 3}$ are the fitted coefficients for the expression representing $\mu_{\text {above }}$.

Note: In this report, units of the mass attenuation coefficients are $\mathrm{cm}^{2} / \mathrm{g}$ with energy given in $\mathrm{keV}$. 
Table A4. Revised $U$ and Pu mass attenuation coefficient parameters (including coherent scattering)

\begin{tabular}{ccc}
\hline & Uranium & Plutonium \\
\hline$\mu_{L 0}$ & - & -0.0056 \\
$\mu_{L 1}$ & 0.0862 & 0.2714 \\
$\mu_{L 2}$ & -2.8288 & -3.4634 \\
$\mu_{L 3}$ & 5.6047 & 6.2145 \\
\hline$\mu_{H 1}$ & 0.1857 & 0.1857 \\
$\mu_{H 2}$ & -3.2325 & -3.2230 \\
$\mu_{H 3}$ & 6.5749 & 6.5761 \\
\hline K-Edge (keV) [22] & 115.602 & 121.791 \\
\hline Line Width (eV) [23] & 96.3 & 104.4 \\
\hline
\end{tabular}

\section{A-6. EVALUATION OF THE MASS ATTENUATION COEFFICIENTS USING THE KED TRANSMISSION SPECTRA}

By inverting the above KED analysis, the mass absorption coefficient for uranium as a function of energy (Section A.1) can be determined from the KED transmission spectra. The normal approach would be to measure the spectra for the uranium solution and compare it to the spectrum from a reference blank. However, by examination of the ratio of count rates from two different uranium solutions the primary error contributors to the attenuation coefficients:

- Bremsstrahlung source energy distribution.

- Dead-time differences: The count rates from the reference blank are several times greater than from the uranium solution.

- Uncertainties in attenuation from system hardware (e.g., collimator thickness, composition, and density).

- Systematic errors in the standard declaration values.

can be largely eliminated.

The mass attenuation coefficients were determined from the ratio of two KED transmission spectra (321 g U/L and $160 \mathrm{~g} \mathrm{U} / \mathrm{L}$ ). Use of the ratio reduces the contribution from systematic errors in the declaration values for our uranyl-nitrate standards. The ratio method for determination of the mass attenuation coefficients is described by the following.

The KED count rate as a function of energy is given by the response function $\mathrm{C}_{\mathrm{KED}}$ :

$$
C_{K E D}(E)=\left(f_{\text {det }} * f_{K E D}\right)(E)+f_{r_{\text {sum }}}(\mathrm{E})+f_{b k g}(\mathrm{E}) \text {, }
$$

where $C_{K E D}$ is the count rate as a function of photon energy,

$f_{\text {det }}$ is the detector response function,

$f_{K E D}$ is the Bremsstrahlung source term for the HKED system, 
$f_{r s u m}$ is the contribution to the spectrum due to random coincidence summing

$f_{b k g}$ is the background not associated with the X-ray generator

In this case, the background term can be set to zero because there are no fission products in the standard solutions so that the count rate ratio, $R(E)$, for two measurements is given as

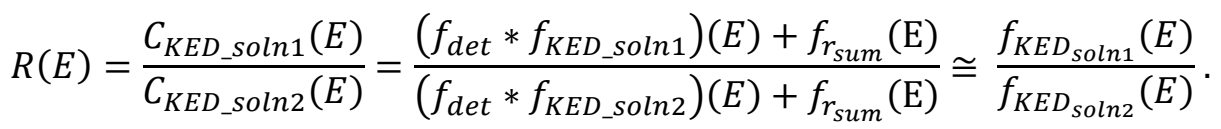

The expression for $f_{K E D}$, from above is

$$
f_{K E D}(E)=\left[f_{\text {Brem }}(E)+f_{\text {small }}(E)+f_{\text {back }}(E)\right] \cdot \delta_{\text {attn }}(E) \cdot \delta_{\text {samp }}(E)+I_{C d}
$$

For energies above $92 \mathrm{keV}$, the ${ }^{109} \mathrm{Cd}$ source term, $I_{C d}$, is approximately equal to zero, so that

$$
R(E)=\frac{\left[f_{\text {Brem }}(E)+f_{\text {small }}(E)+f_{\text {back }}(E)\right] \cdot \delta_{\text {attn_soln } 1}(E) \cdot \delta_{\text {samp_soln } 1}(E)}{\left[f_{\text {Brem }}(E)+f_{\text {small }}(E)+f_{\text {back }}(E)\right] \cdot \delta_{\text {attn_soln } 2}(E) \cdot \delta_{\text {samp_soln } 2}(E)}=\frac{\delta_{\text {samp_soln } 1}(E)}{\delta_{\text {samp_soln } 2}(E)} .
$$

Substituting the expression for $\delta_{\text {samp }}(E), R(E)$ becomes

$$
R(E)=\frac{\mathrm{e}^{-\sum \mu_{m_{i} m} \cdot \rho_{m m_{i}} \cdot d_{\text {vial }}} \cdot e^{-\sum \mu_{Z_{i}} \cdot \rho_{Z_{i}} \cdot d_{\text {vial }}}}{\mathrm{e}^{-\sum \mu_{m_{i} m} \cdot \rho_{m m_{i}} \cdot d_{\text {vial }}} \cdot e^{-\sum \mu_{Z_{i}} \cdot \rho_{Z_{i}} \cdot d_{\text {vial }}}}=\mathrm{e}^{-\sum \mu_{m_{i} m} \cdot \Delta \rho_{m m_{i}} \cdot d_{\text {vial }}} \cdot e^{-\mu_{U} \cdot \Delta \rho_{U} \cdot d_{v i a l}},
$$

where $\Delta \rho_{U}$ is the difference in the uranium density between the two standards, $\Delta \rho_{m m_{i}}$ is the difference in the density of the $\mathrm{i}^{\text {th }}$ component of the solution matrix.

And, finally, the expression for $\mu_{U}(E)$ is

$$
\mu_{U}(E)=-\frac{\ln (R(E))+\sum \mu_{m_{i} m}(E) \cdot \Delta \rho_{m m_{i}} \cdot d_{\text {vial }}}{\Delta \rho_{U} \cdot d_{\text {vial }}}=-\frac{\ln (R(E))}{\Delta \rho_{U} \cdot d_{\text {vial }}}-\frac{\sum \mu_{m_{i} m}(E) \cdot \Delta \rho_{m m_{i}}}{\Delta \rho_{U}} .
$$

Since the change in density of the nitric acid solution with uranium concentration is small $(<0.1 \mathrm{~g} / \mathrm{mL})$ and the mass attenuation coefficients for the nitric acid elements are also small, use of the declared matrix concentrations and the XCOM attenuation coefficients will introduce a negligible error into the measurement of the mass attenuation coefficients for uranium.

The mass attenuation values based on the count rate ratios from two uranium standards (160 and $321 \mathrm{~g} \mathrm{U} / \mathrm{L}$ ) assayed sequentially (to minimize variation in the interrogating X-ray distribution) are shown in Figure A18 along with the accepted values from the XCOM database. For this comparison we have applied a broadening function based on the observed behavior of the HPGe detector rather than attempting to deconvolute the measured spectrum.

In this analysis, the value of $\Delta \mu$ (the change in the mass attenuation coefficient at the K-Edge boundary) used in the traditional KED analysis was determined to have a value of $\Delta \mu=3.518 \pm 0.004 \mathrm{~cm}^{2} / \mathrm{g} \mathrm{U}$ versus the value expected from the XCOM database for $\Delta \mu$ was 3.516. (Note: To our knowledge this is the only direct measurement of the cross section). 


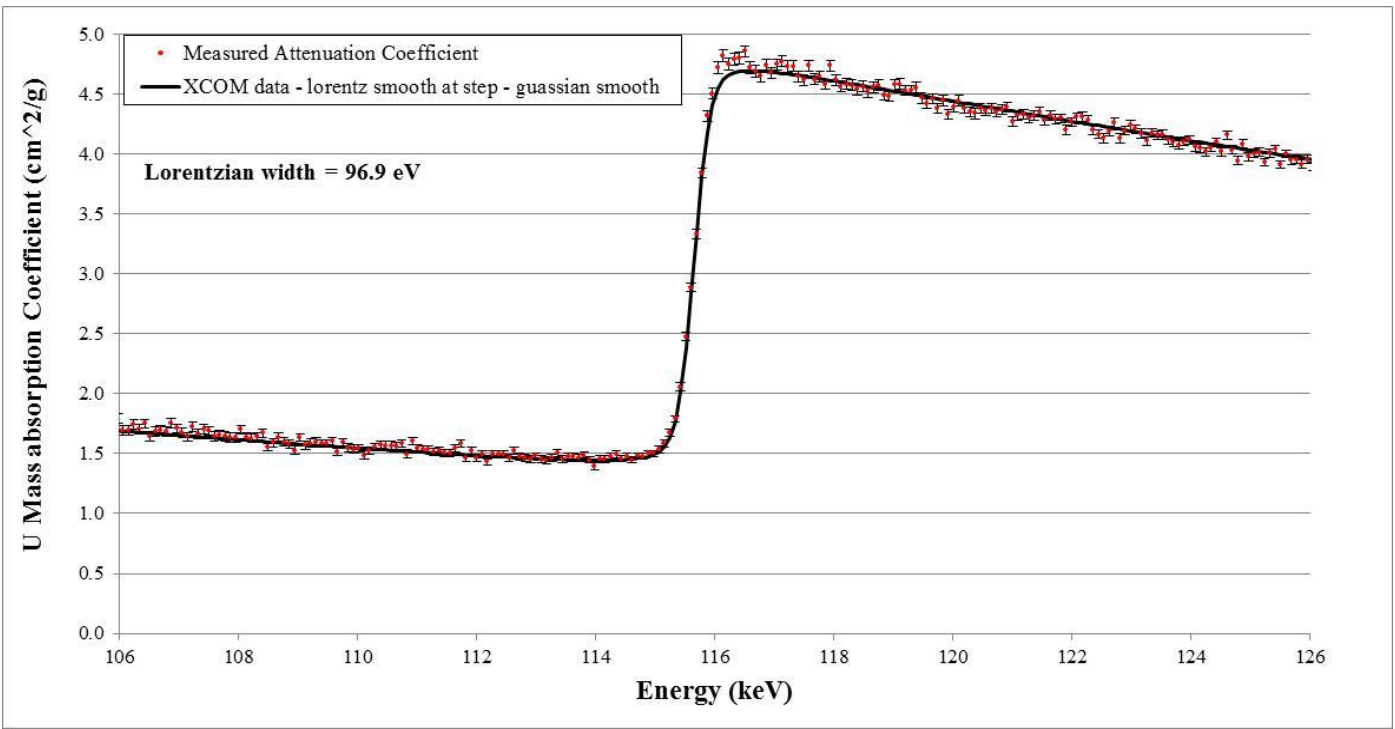

Figure A18. Determination of the uranium mass absorption cross-sections from the KED transmission measurement on a $320 \mathrm{~g} \mathrm{U} / \mathrm{L}$ aqueous solution. This plot compares the Voigt broadened accepted mass coefficient value as a function of energy from the XCOM database with the mass attenuation coefficients determined from our transmission measurements. The deviation between measured and expected reflects the lack of Lorentzian broadening of the high energy side of the edge. 



\section{APPENDIX B. STANDARDS USED FOR THE ORNL HKED TESTING}

The standards described below were produced prior to this project from Oak Ridge National Laboratory uranium and plutonium stock materials dissolved in $3 M$ nitric acid. Concentration values were determined by isotope dilution mass spectrometry and inductively coupled plasma mass spectrometry analysis. Each standard contains $4.99-5.00 \mathrm{~mL}$ of solution contained within an SGN sample vial.

Declared concentrations for aqueous

$\left(\mathrm{HNO}_{3}\right)$ uranium standards

\begin{tabular}{|c|c|c|}
\hline \multirow{2}{*}{$\begin{array}{l}\text { Sample ID } \\
\text { U001 }\end{array}$} & \multicolumn{2}{|c|}{$\begin{array}{l}\text { Uranium } \\
(\mathrm{mg} / \mathrm{mL})\end{array}$} \\
\hline & 1.070 & \pm 0.002 \\
\hline U005 & 5.360 & \pm 0.011 \\
\hline U015 & 16.119 & \pm 0.032 \\
\hline U045 & 48.273 & \pm 0.097 \\
\hline U100 & 107.30 & \pm 0.21 \\
\hline U150 & 160.91 & \pm 0.32 \\
\hline U200 & 214.61 & \pm 0.43 \\
\hline $\mathrm{U} 250$ & 268.21 & \pm 0.54 \\
\hline U300 & 321.91 & \pm 0.64 \\
\hline Water blank & 0 & \\
\hline $\mathrm{HNO}_{3}$ blank & 0 & \\
\hline
\end{tabular}

Declared concentrations and U:Pu ratios for the Aqueous $\left(\mathrm{HNO}_{3}\right)$ uraniumplutonium standards

\begin{tabular}{|c|c|c|c|c|c|c|}
\hline Sample ID & & $\begin{array}{l}\text { canium } \\
\text { 1g/mL) }\end{array}$ & & $\begin{array}{c}\text { Pu } \\
\text { g/mL) }\end{array}$ & \multicolumn{2}{|c|}{ U:Pu ratio } \\
\hline UPu100 & 107.30 & \pm 0.21 & 1.041 & \pm 0.002 & 103.08 & \pm 0.29 \\
\hline UPu150 & 160.91 & \pm 0.32 & 1.566 & \pm 0.003 & 102.74 & \pm 0.29 \\
\hline UPu200 & 214.65 & \pm 0.43 & 2.082 & \pm 0.004 & 103.08 & \pm 0.29 \\
\hline UPu250 & 243.26 & \pm 0.49 & 2.932 & \pm 0.006 & 82.98 & \pm 0.24 \\
\hline
\end{tabular}

The isotopic composition of the aqueous uranium and uranium-plutonium solutions is shown below. The uranium source selected was highly depleted to minimize the alpha activity contained in the standard. The plutonium source material was chosen to minimize alpha activity; the choice was also based upon the availability of the material.

\begin{tabular}{|l|l|l|}
\multicolumn{4}{|c|}{ Uranium isotopic abundances } \\
\hline & Atom \% & Weight \% \\
\hline U-233 & 0 & 0 \\
U-234 & 0.001 & 0.001 \\
U-235 & 0.04 & 0.04 \\
U-236 & 0 & 0 \\
U-238 & 99.96 & 99.959 \\
\hline
\end{tabular}




\begin{tabular}{|l|l|l|}
\multicolumn{3}{|c|}{ Plutonium isotopic abundances } \\
\hline & Atom \% & Weight \% \\
\hline $\mathrm{Pu}-238$ & 0.01 & 0.01 \\
$\mathrm{Pu}-239$ & 93.271 & 93.241 \\
$\mathrm{Pu}-240$ & 6.197 & 6.221 \\
$\mathrm{Pu}-241$ & 0.136 & 0.138 \\
$\mathrm{Pu}-242$ & 0.386 & 0.391 \\
$\mathrm{Pu}-244$ & 0 & 0 \\
\hline
\end{tabular}

The epoxy-matrix standards on loan from Mirion (formerly Canberra Industries) provides us with a sample matrix that does not contain nitric acid. These standards are primarily being evaluated for use as reference standards that can be transported by the inspector or stored at the facility for system verification purposes.

The source material is depleted uranium with concentrations shown below:

Declared concentration for the uranium epoxy standards

\begin{tabular}{|l|lc|}
\hline Standard & \multicolumn{2}{|c|}{$\begin{array}{c}\text { Declared concentration } \\
\text { (mg-U/mL) }\end{array}$} \\
\hline Blank & 0 & - \\
$958-8-1$ & 0.92 & \pm 0.03 \\
$958-8-2$ & 4.07 & \pm 0.05 \\
$958-8-3$ & 10.12 & \pm 0.11 \\
$958-8-4$ & 21.96 & \pm 0.23 \\
$958-8-5$ & 37.67 & \pm 0.38 \\
$958-8-6$ & 74.67 & \pm 1.16 \\
\hline
\end{tabular}

